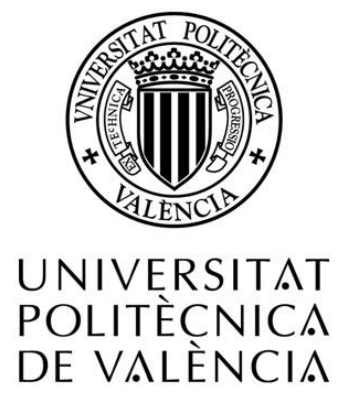

\title{
Design and implementation of nanoantennas on integrated guides and their application on polarization analysis and synthesis
}

\author{
Alba Espinosa Soria \\ Supervised by Prof. Alejandro José Martínez Abiétar
}

DOCTORAL THESIS

Valencia, May 2018 
A mis padres.

Esto es un paso más, gracias por seguir enseñándome a caminar. 


\section{Acknowledgments}

Working on this Thesis has meant for me an unprecedented process of growth and learning. For this reason, I would like to thank Prof. Alejandro Martínez Abiétar for opening the doors to scientific research and then accompanying and encouraging me at all times, helping me to achieve goals that I never thought myself capable of. Also to Dr. Francisco José Rodríguez Fortuño, Pak, your willingness to advise me and resolve so many doubts throughout my career continues amazing me every day. I would like to thank Prof. Nader Engueta for the great opportunity to be part of his team at the University of Pennsylvania, with all the good professional and personal consequences that this has entailed.

Thanks to my NTC colleagues. Thank you, Sara, my dear becaria. Angela, thank you for your freshness. Sergio and Luis, with whom fun and science are not at odds. Fede, for your help. Margue, Fran, Álvaro, Andrés, Raffaelle, Julio, Kike, Javi, Alex, Pau, Anita, Bea, Laura, thank you for always being there when you are needed, both inside and outside the office. Carlos, Rubén, Elena, for being a great reference. Thanks to the fabrication team, especially to Amadeu, thank you for everything. And to the other colleagues in the NTC, to everyone, because work becomes more pleasant when you feel at home.

Thanks to my UPenn colleagues. Max, without you nothing would be the same. Mario, for your help and supervision. Chen-Ta, Prathmesh, Victor, Diego, Brian, Koby and Nasim, for welcoming me so warmly. To my friends in Philadelphia: Oriana, Menchie, Rucha, Camilo, Sam, Ele, Martha, Aizhan, for sharing with me so many moments and new experiences.

Gracias a mis amigos. Javi, Xus, Juan y Marc, por seguir a mi lado. Yoli, Mayte, Laura, Anabel y Lorena, por el gran equipo que formamos y lo necesario que es para mí.

Gracias toda mi familia. Especialmente a mi prima Ana, cuyo apoyo y compañía son tan valiosos; y a Merche y Juan, por cuidarme como una hija.

Y sobre todo gracias a mi hermano Miguel, porque te admiro y te necesito. A Sergio, por hacerme completamente feliz. Y a vosotros: Papá, Mamá, sois artífices de cada logro, sois mi ejemplo a seguir. 


\section{Contents}

Acknowledgments..................................................................................................

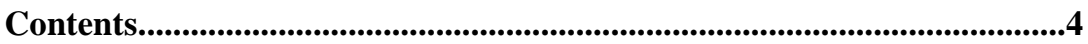

Chapter 1 Introduction ......................................................................................6

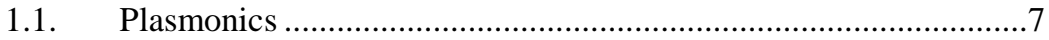

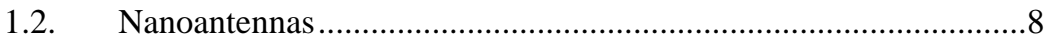

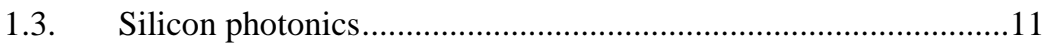

1.4. Combining plasmonics and silicon photonics ...............................12

1.5. Motivation and objectives ...........................................................16

Chapter 2 Guided modes in silicon waveguides: longitudinal components and transverse spin .....................................................................19

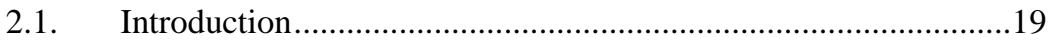

2.2. Description of the concept ……………………......................20

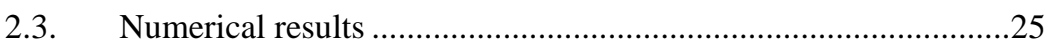

Chapter 3 Embedding plasmonic nanoantennas in silicon waveguides .29

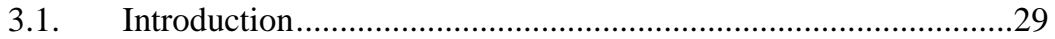

3.2. Description of the concept ..........................................................

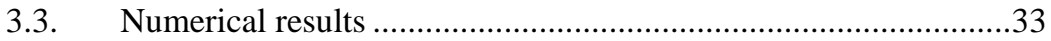

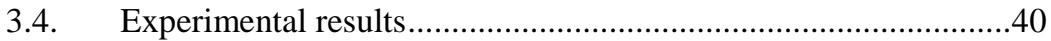

Chapter 4 Coherent control of plasmonic nanoantennas........................45

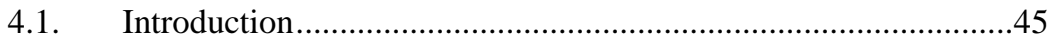

4.2. Coherent control of absorption, scattering and transmission ........48

4.2.1. Description of the concept....................................................48

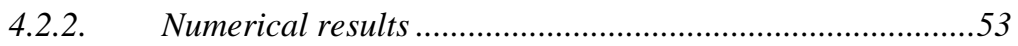

4.2.3. Experimental results..........................................................59

4.3. Coherent synthesis of scattering polarization................................63

4.3.1. Description of the concept.................................................63

4.3.2. Numerical results ..............................................................65

4.3.3. Experimental results.......................................................66 
Chapter 5 Optimal Stokes polarimetry implemented on silicon nanoantennas ....................................................................................................69

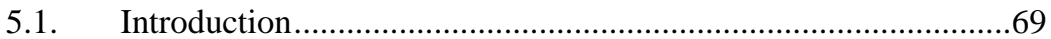

5.2. Description of the concept ....................................................... 70

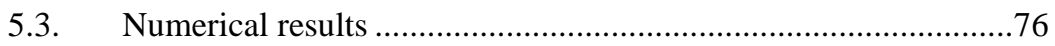

5.3.1. Optimization of the nanopolarimeter ...................................83

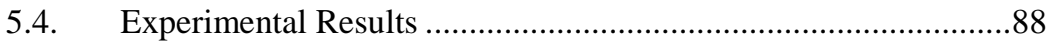

Conclusions and future work .............................................................................98

Methods ..................................................................................................102

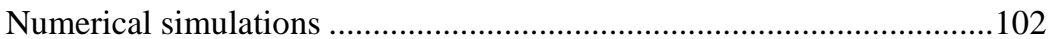

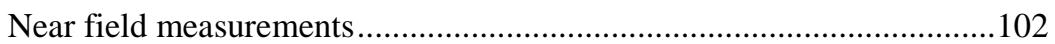

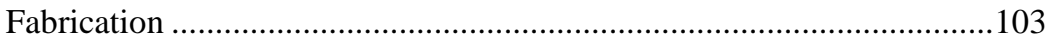

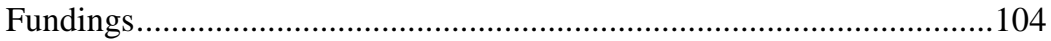

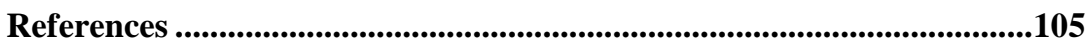

Author's merits.............................................................................................119

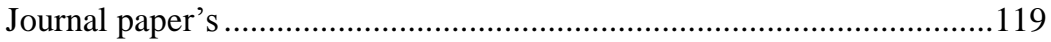

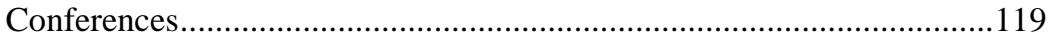

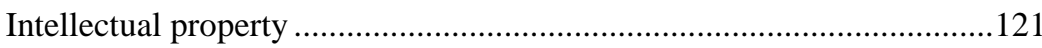

Abstract ................................................................................................122

Resumen ......................................................................................................123

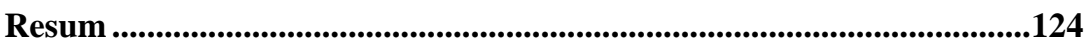




\section{Chapter 1 Introduction}

The interaction of light with subwavelength nanostructures is at the heart of different current scientific hot topics as plasmonics and nanoantennas. Research in these disciplines during the last decade has given rise to new, powerful concepts providing an unprecedented degree of control over light manipulation at the nanoscale. This unprecedented control could involve the introduction of nanostructures to increase the capabilities of light processing in current photonic integrated circuits, which traditionally rely only on dielectric materials with element sizes larger than the light wavelength. Amongst the different available platforms, silicon photonics is expected to become mainstream, since manufacturing using well-established CMOS processes enables the mass production of low-cost photonic devices. This Thesis presents recent advances showing how new concepts arising from plasmonics and nanoantennas research can be useful in many applications, that could bring a breakthrough in photonic integrated circuits.

The present chapter discuss the benefits of introducing plasmonic and nanoantennas concepts into a silicon photonics integrated platform, which means the final objective of the structures addressed in this Thesis. It is a review of existing works in this direction and a discussion of how this hybrid approach can lead to the improvement of current photonic integrated circuits enabling novel and disruptive applications in photonics.

In chapter 2 it is demonstrated the existence of large longitudinal fields in the evanescent field region of silicon waveguides, which are the links in the proposed silicon-based integrated structures. This fact makes a strong difference in comparison with typical excitation of plasmonic nanostructures using free-space plane-wave-like light, where the transverse component is dominant, what makes this study essential to properly design the structures.

In chapter 3, it is demonstrated how single plasmonic nanoresonators can be efficiently coupled to silicon waveguides. This approach can bring several advantages: for silicon photonics, since it would enable subwavelength components to be used in sensing or switching, therefore allowing for denser integration; and for plasmonics, since this 
approach would enable scanning multiple isolated plasmonic nanostructures in parallel and in real-time.

In chapters 4 and 5, two different implementations of metallic as well as dielectric nanoantennas are proven on silicon photonics. Chapter 4 shows experimentally the coherent control of an isolated plasmonic nanoantenna with foot-print much smaller than the wavelength. The nanoantenna is built on a subwavelength gap separating two silicon waveguides, which provide the guided paths for counter-directional illumination with controlled phase. Using a transfer-matrix formalism, a simple model that explains qualitatively the main features of the system is developed, showing that it is possible to modulate the optical intensity of the scattered and transmitted signals by changing the phase different between the feeding paths. Chapter 5 shows that, by means of spin-orbit interaction of light, such nanoantennas enable polarization manipulation at the nanoscale in both transmission and reception schemes, which could be used for building polarization-selective elements on silicon PICs. In particular, it is shown how to build Stokes nanopolarimeters with optimal performance.

\subsection{Plasmonics}

Plasmonics is the science that studies surface plasmon (SP) propagation along metal surfaces (see Figure 1.1(a)) at visible and near-infrared (NIR) wavelengths [1]. A key property enabled by SPs is the strong light confinement well beyond the diffraction limit in metallic nanostructures (see Figure 1.1(b)) via excitation of localized SP resonances (LSPRs) [2]. This property can be used to build subwavelength-sized plasmonic nanoresonators extremely sensitive to tiny variations in their surroundings (useful in sensing) [3] or plasmonic nanoantennas that efficiently convert confined into radiative fields and vice versa [4]. Isolated metallic nanostructures can also be arranged into 2D or 3D artificial structures named metasurfaces and metamaterials, respectively, which can provide a number of intriguing phenomena such as negative refraction [5], cloaking [6], optical magnetism [7] or phase manipulation [8,9,10]. Such amazing abilities for manipulating light at the nanoscale have pushed plasmonics, nanoantennas and metamaterials to become research hot-topics nowadays, being strongly related by the fact that their properties ultimately rely on the ability to tailor the optical response (scattering, absorption, polarization, etc) of subwavelength metallic 
nanostructures - either isolated $[3,7,11,12,13,14]$ or forming compact arrays $[9,15]$ - at will.

(a)

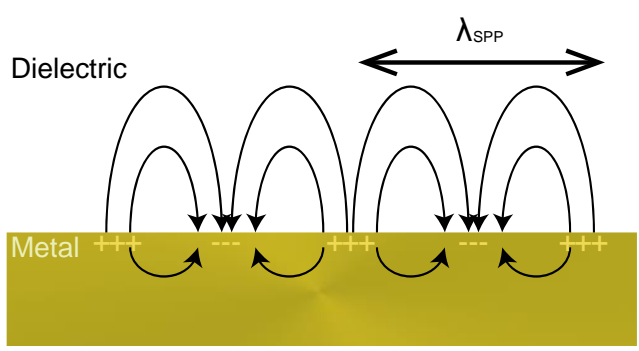

(b)

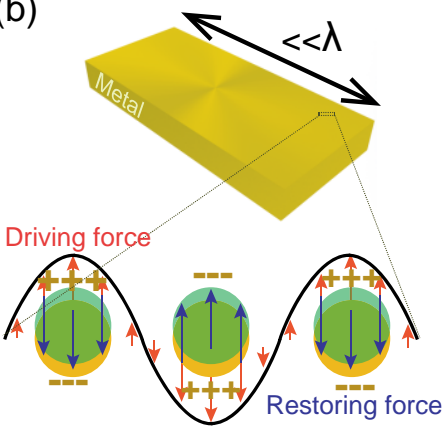

Figure 1.1 (a) Surface plasmon propagation at a flat dielectricmetal interface. (b) Plasmon representation as a plasma movement around the geometrical center of a nanoparticle.

The use of metals at optical frequencies comes at a price: high absorption losses prevent efficient wave-guiding at long distances ( $\mathrm{cm}-$ scale). Another important drawback resulting from the subwavelength size of metallic nanostructures is the difficulty of characterizing them when isolated. Indeed, simultaneous but independent excitation and measurement of multiple nanostructures becomes challenging, requiring near-field approaches, which is a huge limitation in applications that benefit from multiplexing such as, for instance, biosensing. Finally, typical metals - mainly gold ( $\mathrm{Au}$ ) and silver (Ag) - and some of the fabrication processes (such as focused ion beam) are not compatible with standard semiconductor fabrication techniques, mainly CMOS, which hinders bringing plasmonics out of the lab [16].

\subsection{Nanoantennas}

It is well known that, besides providing strong field confinement or large absorption at LSPR frequency, plasmonic nanostructures can also be used as efficient transducers between nanoscale sources and freespace radiation. In other words, they can bridge near and far fields [4, 17,18]. This transducing behavior resembles that offered by traditional antennas in the radiofrequency (RF) domain - where transmission lines and waveguides can exhibit an extremely subwavelength crosssection — but at scales that are several orders of magnitude smaller [17]. 
As a result, such plasmonic nanostructures have been termed optical antennas [18] or nanoantennas [19].

So far, many suggested applications of nanoantennas rely on the high intensity of their near fields, known as hot-spots (Figure 1.2(b)), achieved under far-field excitation, enabling many different enhanced phenomena such as photodetection [20], gas sensing [21], harmonic generation [11], or vibrational spectrometry [22]. However, this Thesis is focus on a more traditional definition of an antenna as that of a device which converts radiated waves into guided waves, and vice versa. It is important to notice that this definition, commonly found in electrical engineering textbooks [23, 24], does not necessarily involve a subwavelength size in the guided domain (Figure 1.2(a)). This way, antennas are used to interconnect wired and wireless networks in an efficient way thus bridging the best of two worlds: signals propagating through free space can simultaneously reach multiple receivers, even if they are at very long distances, without the need for cable deployment; whilst signals in the guided domain can be reliably processed at very high speeds using well-matured technologies.

This traditional concept of antenna as a guided-to-radiated wave transducer can also be applied in the optical domain, where the vast knowledge from RF antenna engineering can be exploited. In the radiated domain, light is used in displays, imaging, communication, and optical manipulation, among many other well-known applications. In the guided domain - for example, in a silicon PIC, low-loss dielectric waveguides can be used to carry optical information at different parts of a chip and serve as the basis for information processing at speeds ultimately reaching the $\mathrm{THz}$ scale (notice that processes such as demultiplexing [25], switching [26] or amplification [27] can be attained in silicon photonic integrated circuits).

The applications enabled by bridging both worlds of radiated and guided light are many. For instance, the possibility of phase tuning the feeding signals of nanoantennas at $\mathrm{THz}$ speed would allow exciting applications such as ultra-fast beam-forming and polarization control of light. Proof of principle demonstrations include arrays of hundreds of dielectric optical nanoantennas used for beam-forming [28], and synthesis of light polarization through the amplitude and phase of the feeding signals on antennas with multiple inputs [29]. Both approaches employ a silicon photonics platform. However, the diffraction limit establishes that dielectric antennas and their feeding waveguides will always have minimum dimensions comparable to the light wavelength. 
In this sense, dielectric optical antennas are not truly 'nano' in scale (Figure 1.2(c)).
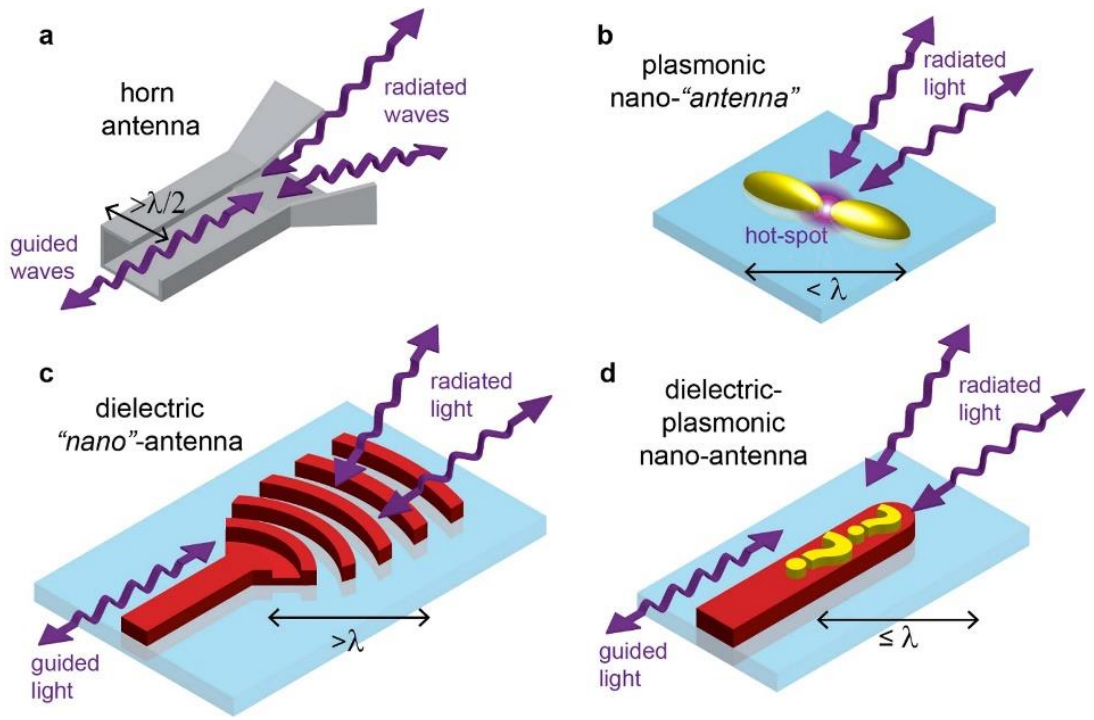

Figure 1.2 Nanoantennas as a bridge between wired and wireless domains. (a) An RF horn antenna converts from guided into radiated fields and vice versa in an efficient way. Notice that the waveguide dimensions can be comparable to the wavelength; (b) a plasmonic nanoantenna produces a strong subwavelength field spot when illuminated from free-space, and acts as a transducer between a localized point source or absorber and radiated light; (c) a dielectric optical antenna fed by a dielectric waveguide [28] behaves as a classic RF antenna but its size is of the order of - or larger than - the wavelength; (d) hybrid approach: a subwavelength-sized plasmonic nanoantenna is fed by a dielectric waveguide in order to inherit the best properties of the two worlds. The nanoantenna shape and size should be designed to ensure impedance matching to the waveguide and directional emission in the desired direction.

The relatively large size of dielectric optical antennas results in a significant drawback: when forming arrays, the many-wavelengths period results in unavoidable high-order beams [28]. Alternatively, designing a small dielectric optical antenna comes at the cost of poor transducing efficiency (low effective area) [29]. The diffraction limit in dielectrics therefore imposes a severe trade-off limitation. In order to overcome the diffraction limit and achieve a subwavelength optical nanoantenna without compromising the transducing efficiency, the 
attention can be turned to plasmonic resonators. This approach, which closely mimics the use of metallic antennas in RF, is similar to that discussed in plasmonics section, but in this case the interest is focused on the scattering properties of the plasmonic nanostructures. As mentioned before, most plasmonic nanoantennas in the literature are designed to achieve near-field hotspots, with very interesting applications (Figure 1.2(b)), but without considering the guided domain. Here the focus is in those works on plasmonic nanoantennas that follow the traditional definition of antenna and thus include the guided domain-including the use of RF concepts such as input impedances [30, 31]. This approach enables exciting plasmonic applications such as wireless optical communications [32] and tunable light beam-forming [33], closely matching those of RF antennas. Beam-forming arrays show no side-lobes thanks to the subwavelength period of the plasmonic nanoantenna elements [33]. Unfortunately, plasmonic waveguides display high propagation losses and, therefore, are currently not generally suitable as long-range information highways.

\subsection{Silicon photonics}

In parallel to plasmonics, recent years have witnessed the success of silicon photonics technology, from the first experimental demonstrations in the 90s to becoming the mainstream technology for photonic integrated circuits. The key advantage of silicon photonics over competing approaches (such as III-V semiconductor heterostructures) is the feasibility for mass-scale production of low-cost chips integrating photonic and electronic components made by existing semi-conductor fabrication techniques (such as CMOS) [34,35,36,37]. Silicon photonics is now a mature technology and silicon integrated circuits fabricated on silicon-on-insulator (SOI) wafers are currently being exploited for label-free biosensors $[38,39,40]$ and high-speed data communications $[41,42]$ amongst other applications, even with commercial products in the market $[43,44]$.

Some of the properties inherent to silicon integrated circuits would enable overcoming the existing drawbacks related to plasmonics and nanoantennas introduced above. In silicon photonic circuits, light propagates along low-loss silicon waveguides at NIR wavelengths or along silicon nitride $\left(\mathrm{Si}_{3} \mathrm{~N}_{4}\right)$ waveguides at visible wavelengths. The transverse size of these waveguides is diffraction limited to about $(\lambda / 2 n)^{2}$, being $n$ the refractive index of the waveguide core. This means 
that the confinement is not deeply subwavelength, as plasmonic waveguides may allow, but in contrast they exhibit propagation losses below $1 \mathrm{~dB} \mathrm{~cm}^{-1}$ [45], which enables interconnection of processing elements well-spaced (cm-scale or beyond) on an integrated circuit. In this sense, it is possible to think about a hybrid architecture in which dielectric waveguides are used for guiding signals while plasmonic elements or nanoantennas, with their novel features, realize several functionalities (see Figure 1.3). This hybrid approach also permits the parallel excitation and measurement of multiple nanostructures by coupling them to several silicon waveguides, which can all be fed simultaneously but detected independently. Finally, realization of plasmonic or nanoantennas devices on silicon technology could pave the way towards the industrialization of such technologies. To this end, the use of silicon-compatible materials and processes becomes mandatory.

An appropriate solution would be the hybrid architecture: using plasmonics resonators as nanoantennas while keeping dielectric waveguides as the feeding elements (or 'wired' network). This brings with it all the advantages of dielectric waveguides named above regarding low-loss and processing abilities, thus gaining the best of each world (Figure 1.2(d)): the subwavelength dimensions and strong field localization of plasmonic nanoantennas with the lossless guiding and fast processing properties of wavelength-size dielectric feeding waveguides. Some preliminary works have shown advances in this direction. For instance, on [46] a directional plasmonic Yagi-Uda nanoantenna is placed on a dielectric waveguide, allowing directional coupling between guided and radiated modes, and on [47] a plasmonic nanoantenna array on a dielectric waveguide is used for electrically controlled steering of radiated light. Although in the latter case the antenna array does not have a subwavelength period, it has only a few side lobes thanks to the smaller size of the plasmonics elements compared to dielectric nanoantennas.

\subsection{Combining plasmonics and silicon photonics}

Considering all the above, silicon photonic integrated circuits could benefit from the introduction nanoantennas and plasmonic elements. For instance, the diffraction limit prevents further miniaturization of dielectric circuits below the light wavelength [48], which could be highly alleviated by employing deep-subwavelength plasmonic-based building blocks $[2,49]$. The relatively weak and slow nonlinear effects 
in silicon result in large structures operated at high energies for alloptical processing, with speeds limited to some tens of GHz [50]. Giant and ultrafast nonlinearities exhibited by plasmonic nanostructures [51,52] and metamaterials [53] could provide a way towards realizing THz-speed all-optical switching on silicon PICs. Sensitivity to the environment provided by LSPRs in metals surpasses that exhibited by dielectric structures, so the performance of silicon photonic biosensors could also be improved by using plasmonics. Finally, nanoantennas could be employed as ultra-compact elements for on-chip and interchip wireless interconnects [54] as well as for imaging purposes. Hence, it is clear that enormous benefits may arise from the use of plasmonics and nanoantennas on silicon photonic circuits (Figure 1.3)

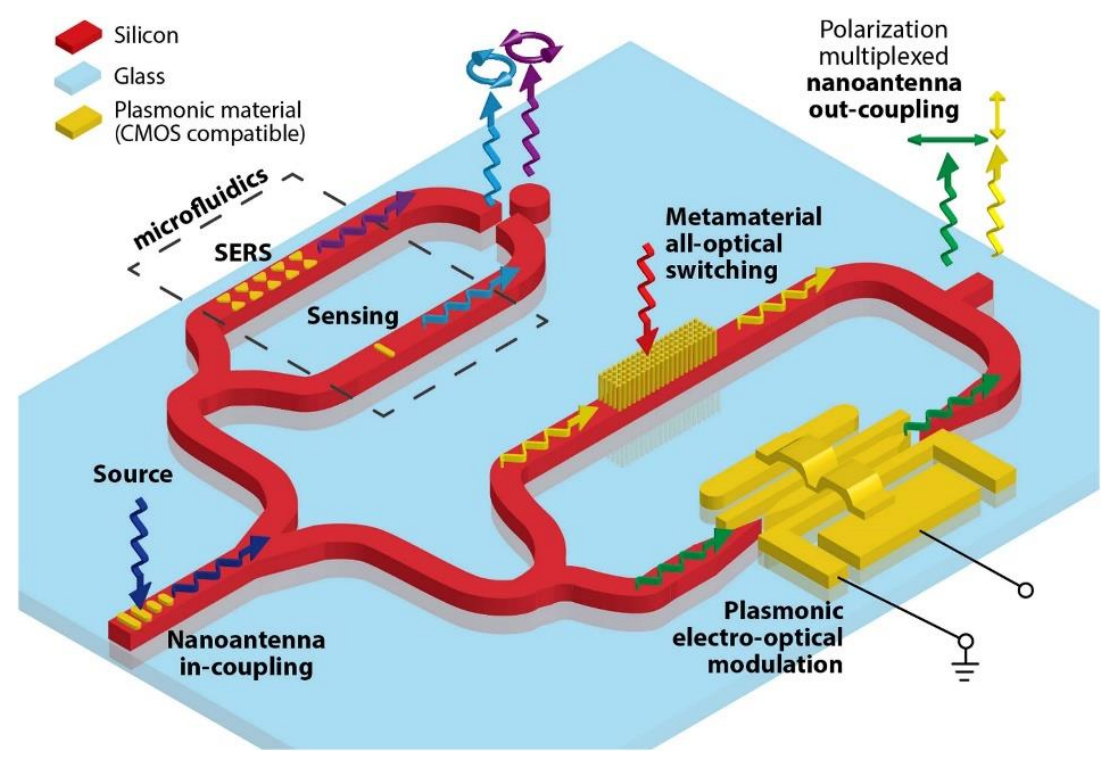

Figure 1.3 Scheme of the proposed hybrid architecture. Silicon waveguides with negligible losses interconnect compact and efficient plasmonic and metamaterial elements. The left-part of this silicon photonic integrated circuit includes plasmonic elements for enhanced Raman [55] and refractomeric biosensing [55] of a certain fluid. The right-part of the PIC includes electrooptical (with a plasmonic slot Mach-Zehnder interferometer [56]) and all-optical (with a rod-based hyperbolic metamaterial [58]) modulation/switching. Nanoantennas are used as transducers between the outer world and the waveguides: the input port includes a highly directive Yagi-Uda [46] for highly directional 
coupling and polarization-dependent output nanoantennas for polarization multiplexing of the output radiated light.

In the hybrid architecture previously described, there is a silicon PIC with low-loss silicon (or silicon nitride) waveguides to transport light between different regions of the chip and plasmonic, nanoantenna or metamaterial elements in which the light is processed (for example, biosensing or switching). Such elements should be placed in close proximity to the waveguides to ensure efficient interaction between them and the guided light. In non-guided configurations in which light coming from free-space impinges from outside the chip (top-side illumination) on a plasmonic element it can be assumed that the excitation is a plane wave with an electric field that defines the polarization. Indeed, this electric field can be adequately chosen to excite or not a certain LSPR. However, the situation becomes very different when the excitation (and also the collection of the light scattered by the plasmonic element) is realized via high-index waveguides. In this case, the guided light can no longer be approximated by a plane-wave because of the strong confinement by the large index contrast. Indeed, in Chapter 2 it is demonstrated that guided modes show a large longitudinal component of the electric field accompanied by a transverse spin [59], which does not exist in planewave-like excitation. Figure 1.4 shows the main transverse $\left(\mathrm{E}_{\mathrm{x}}\right.$ for TElike mode and $\mathrm{E}_{\mathrm{y}}$ for TM-like mode) and longitudinal $\left(\mathrm{E}_{\mathrm{z}}\right)$ electric field components as well as the local helicity of the first two modes of a strip silicon waveguide. If the TE-like mode of the waveguide is considered for excitation of a plasmonic nanostructure, only if the latter is very small (100 nm or less) and symmetrically placed with respect to the yzplane, the longitudinal component vanishes and the excitation can be considered similar to the case of an incident plane wave.

However, as long as the nanostructure gets larger or placed in a region with a non-negligible longitudinal component, the effect of this field component has to be taken into account when considering its excitation from the guided field. Indeed, since $E_{x}$ and $E_{z}$ exhibit a $\pi / 2$ phase shift between them, such regions display local elliptical polarization (see right column in Figure 1.4), which will result in spin-orbit interaction effects $[59,60,61]$. This interaction, as well as the resulting interesting possibilities of spin-controlled directional guiding phenomenon, are considered in depth in Chapter 5. 


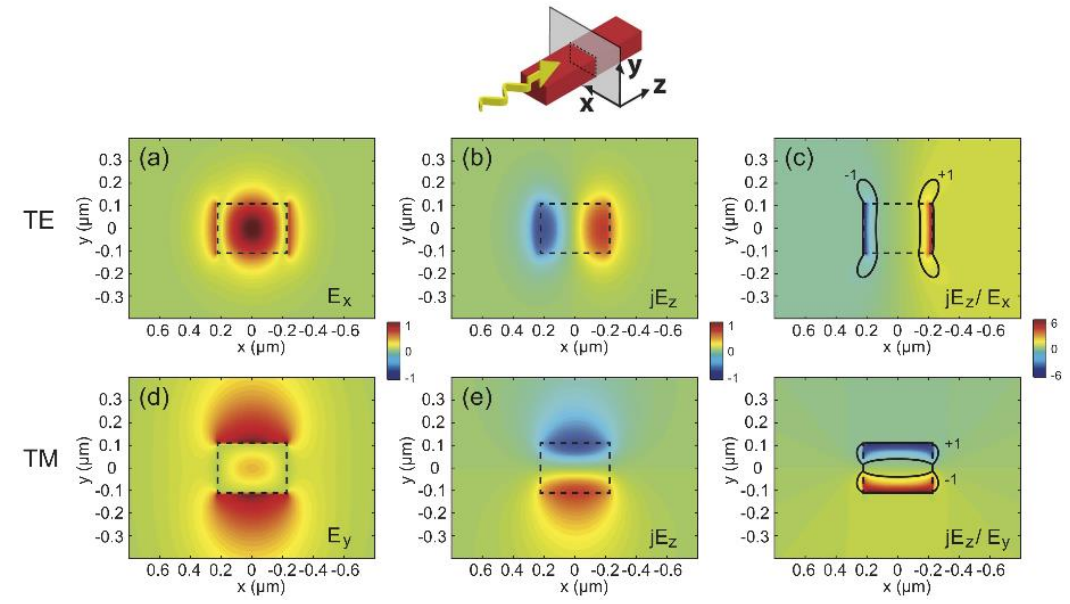

Figure 1.4 Electric field components of the two fundamental guided modes (top: TE-like or even mode; bottom: TM-like or odd mode) of a silicon strip waveguide with $400 \times 220 \mathrm{~nm}^{2}$ rectangular cross-section at $\lambda=1,550 \mathrm{~nm}$. (a,d) Fundamental transverse electric field component responsible for coupling and excitation from external optical fibers; (b,e) longitudinal electric field component $\left(j \mathrm{E}_{\mathrm{z}}\right)^{1}$. (c,f) Local helicity obtained as the ratio between the longitudinal and transverse component; local polarization turns out to be elliptical except for the highlighted contours where it is purely circular.

In Chapter 3 it is considered small plasmonic nanostructures being symmetric with respect to the yz-plane so that the effects related to the longitudinal field component can be disregarded when exciting the LSPR. In particular, the objective is coupling between an isolated subwavelength metallic nanostructure and a dielectric strip waveguide. This configuration may be especially relevant for on-chip biosensing, with the important advantage of being able to drive and measure multiple nanostructures coupled to different waveguides in parallel and in real time, even using different wavelengths for each one, which is very complicated in top-side illumination architectures. The simplest way to do so is merely placing the plasmonic element on top of a silicon waveguide, where the nanostructure is placed in a region of evanescent field of the guided mode so the interaction between the nanostructure LSPR and the guided field turns out to be weak. Even delocalizing the

\footnotetext{
${ }^{1}$ In this Thesis, phasors are taken to follow the engineering convention time-dependence $e^{+j \omega t}$.
} 
guided field by, for example, reducing the waveguide thickness, maximum values of the coupling efficiency (calculated from the extinction ratio, which is obtained as the ratio between the scattered plus the absorbed power to the incident guided field) are relatively small, which means that most of the guided field does not 'see' or interact with the plasmonic nanostructure and is therefore not 'processed'. Such weak interaction can be enhanced by placing several nanostructures on the waveguide, doing so the total length is increased so some of the main features of such nanostructures are missing: the extreme miniaturization to levels beyond the diffraction limit of isolated nanostructures and the possibility to measure their individual response (useful for example in single molecule detection [2]). Therefore, it would be highly desirable to find other ways to place a single nanostructure in order to increase the interaction between the nanostructure resonance and the guided field.

In high-index waveguides, the maximum field intensity of the guided wave is located inside the waveguide core. Therefore, embedding the plasmonic nanostructure inside the waveguide core at the right height would ensure maximum interaction, as recently proposed and numerically analysed [62]. Although this approach achieves strong interaction, it requires digging a relatively deep hole with identical shape and size as that of the plasmonic nanostructure, which may become challenging mainly for nanostructures with complex shapes. An alternative choice that is proposed and demonstrated experimentally in Chapter 3 and Chapter 4 is to create a subwavelength gap in the waveguide core and place the plasmonic nanostructure inside it, at a height ensuring maximum interaction. The field components in the gap region are quite similar to those in a perfect waveguide so longitudinal fields components may play a role [62]. However, thanks to the study carried out in Chapter 2 it can be assumed that, for sufficiently narrow nanostructures, the interaction is governed by the main transverse components of the guided field, being the excitation similar to the case of free-space light incidence.

\subsection{Motivation and objectives}

Yet there is still a lot of work to be performed. For instance, an appropriate matching between dielectric waveguides and plasmonic antennas is mandatory in order to deliver a maximum amount of power to the radiating element whilst minimizing its absorption (Chapter 3 and Chapter 4). The dielectric waveguide could also be modified in 
such a way that the guided field excites different multipoles in the plasmonic nanoantenna resulting in a highly directional beam, a key property for many applications [12,63,64]. Finally, following the miniaturization trend in photonics, it is desirable plasmonic nanoantennas to be smaller than the diffraction limit but with an acceptable loss in efficiency, like in the miniaturization of conventional RF antennas. Applying the traditional definition of antennas to the nanoscale, the broad knowledge of antenna theory could lead to unforeseen applications in optics. For instance, an interesting case in which concepts initially developed in plasmonics can be exploited in silicon photonic nanoantennas is the novel idea of evanescent wave spin-controlled directional guiding [34, 59,64,65,66,67]. This is based on the existence of a large longitudinal component of the electric field of the guided mode (Chapter 2), which is antisymmetric with respect to one of the planes bisecting the waveguide (see Figure 1.4). As already mentioned, this in general results in an elliptical polarization of the local electric field, whose spinning direction is uniquely locked to the propagation direction of the mode (Figure 1.4(c) and (f)). This enables spin-orbit interactions when a scatterer is asymmetrically placed in the waveguide proximity, allowing the use of circular polarizations of topside illuminating light (carrying spin angular momentum) to selectively excite propagating modes in the waveguide in a polarization-dependent way. This concept was originally exploited in particles near surfaces $[14,68]$ and near optical fibers [69], but the effect is universal and can be easily extended to silicon photonic waveguides [29]. Indeed, it provides a unique opportunity to introduce polarization control into silicon photonics via asymmetrically placed antennas. While conventional nanoantennas radiate and receive light of a fixed polarization, waveguide-coupled optical nanoantennas that sort the different polarization components of incident light along opposite guided paths can be designed by employing the concepts of spindirection locking. The concept can also be generalized to work with light polarizations other than circular, by using symmetry considerations, for instance enabling linear polarization sorting in silicon integrated nanoantennas [29,70]. Notably, the inverse concept can also be exploited, as was done in plasmonics [14], to achieve optical nanoantennas with multiple inputs that can synthetize desired radiated polarizations [29]. When combined with the possibility of active phase and amplitude modulation in integrated waveguides, the coherent control of the absorption and scattering of metallic nanoantennas integrated in a silicon waveguides becomes achievable (Chapter 4,4.2). Moreover, these multiple input/output nanoantennas could potentially 
lead to polarization synthesis (Chapter 4,4.3) and analysis (Chapter 5), with applications in integrated ellipsometers [71], communications, quantum optics [72], and magnetic storage applications [73]. 


\section{Chapter 2 \\ Guided modes in silicon waveguides: longitudinal components and transverse spin}

In this chapter, numerical simulations have been performed to obtain the electric field components of the fundamental guided modes of two waveguides typically used in silicon photonics: the strip and the slot waveguide. It is showed the relation between transverse and longitudinal field components, the transverse spin densities and other important parameters, such as the longitudinal component of the socalled Belinfante's spin momentum density (BSMD). By asymmetrically placing a circularly-polarized point-like dipole source in regions showing local circular polarization, the guided mode is excited unidirectionally via spin-orbit coupling. In contrast to metal plates supporting surface plasmons, the multimode behavior of silicon waveguides results in different spin-orbit coupling properties for each guided mode.

\subsection{Introduction}

It has been recently shown that, in contrast to propagating waves in free space exhibiting longitudinal spin, evanescent waves support transverse spin orthogonal to the wave vector $[59,74,75]$. This property of evanescent waves can be generally considered as a manifestation of the quantum spin Hall effect of light [65]. Such transverse spin is the ultimately responsible for the spin-controlled unidirectional excitation (SCUE) of guided waves [14,66,68,69,70,71,76,77,78,79], a phenomenon that has its origin in the spin-orbit coupling taking place when a circularly-polarized subwavelength dipole is placed in the evanescent-wave region of a guided mode. The spin-momentum locking inherent to evanescent waves $[65,80]$ enables to completely switch the propagation direction of the excited guided mode by merely changing the spin of the exciting dipole $[59,69,77]$. By reciprocity, the spin of the wave scattered by a point-like defect placed in the evanescent-field region can be switched by simply reversing the direction of the guided wave [14,29]. This phenomenon, which takes place at any wavelength regime (for instance, at microwave frequencies 
$[76,81])$, may have especial relevance in nano-optics, since it ultimately enables mapping the light spin into a propagating pathway.

Remarkably, longitudinal components of the electric field also take place in propagating fields when tightly confining light via strong focusing [82] or total internal reflection in dielectric structures such as microresonators [83] or waveguides [61, 84]. Although there have been some preliminary demonstrations of SCUE of guided modes in silicon waveguides [70,77], a detailed analysis of the electric field components of the guided modes and its relation spin-orbit related phenomena is still lacking.

All this is a good reason to be interested in the analysis of the electric field components of the guided modes of two common silicon waveguides, the strip and the slot waveguide, at telecom wavelengths. In this chapter it is obtained cross-sectional maps of the relationship between transverse and longitudinal field components and the transverse spin densities and the longitudinal component of the socalled Belinfante's spin momentum density (BSMD) [82]. It is shown that, unlike surface plasmons propagating on infinite metal surfaces, in which the local polarization is always elliptical, silicon waveguide modes show points in which the projection of the electric field vector on a plane containing the propagation direction exhibit purely circular polarization. Moreover, such points can be found outside (in the evanescent region) as well as inside the waveguide core. By placing a circularly polarized point-like dipole source at such points, it is observed SCUE of the guided mode via spin-orbit locking, being the propagating direction switched just by changing the spin of the exciting dipole. Remarkably, it is shown that each guided mode displays a different transverse spin both inside and outside the waveguide core. This results in completely different excitation conditions to achieve unidirectional guiding for each guided mode.

\subsection{Description of the concept}

In this section, a silicon strip waveguide with rectangular cross-section is considered first, which is typically used in silicon photonics for guiding and processing light at telecom wavelengths. It is assumed that the silicon core is surrounded by air, although similar results could be obtained if other low-index materials such as silica or a polymer were considered as cladding. The waveguide axis is along the $z$-direction. Notice that, unlike in photonic crystal waveguides where the polarization distribution also changes along the waveguide axis [85], 
the strip waveguide exhibits continuous translational symmetry along this axis.

Therefore, the polarization properties will not change with the $\mathrm{z}$ coordinate so the study is restricted to the transverse $z=0$ plane. The propagating guided modes are characterized by three electric field components with a certain amplitude profile, $E_{x}(x, y), E_{y}(x, y)$ and $E_{z}(x, y)$, and a real wave vector $k_{z}=2 \pi n_{e f f} \lambda$ where $n_{e f f}$ is the effective index of the mode and $\lambda$ is the free-space wavelength. The fundamental even mode is considered, usually called TE-like mode, characterized by a strong electric field component along the $x$ direction. Indeed, this mode is typically excited in experiments by using an external light beam polarized in such direction. In waveguides with large-size core and low-index contrast, such as standard single-mode optical fibers, the longitudinal component of the electric field is negligible. However, as mentioned before, high-index contrast waveguides with strong confinement leads to considerable values of $E_{z}$. Unlike waveguides with cylindrical symmetry [69], there is not an exact analytical solution for the guided modes in the strip waveguide. Therefore, the fundamental even mode distribution has been calculated numerically by using the finite-element method (see Methods). In the calculations, a wavelength of $\lambda=1,550 \mathrm{~nm}$, a grid size of $5 \mathrm{~nm}$ and a total simulation domain of $3 \times 3 \mu \mathrm{m}$ are considered. Figure 2.1(a) shows a cross-sectional map of the amplitude of the $z$-component of the electric field multiplied by the imaginary unit $\left(j E_{z}\right)$ for propagation of the guided mode along $+z$. Throughout this study, the electric field components are normalized with respect to the value of $E_{x}$ at the origin of coordinates. It can be seen that the $E_{z}$ field is asymmetric with respect to the $x=0$ plane, being its magnitude comparable to $E_{x}$ but with a phase shift of $\lambda / 2$ between them. To better appreciate the relation between the longitudinal and transverse components of the electric field, Figure 2.1(b) and (c) show the maps of $j E_{z} / E_{x}$ and $E_{y} /\left(j E_{z}\right)$ respectively. Notice that, for each case, the order of the field components is chosen when performing the ratio in order to get a better visual representation. In contrast to surface plasmons on metallic surfaces or guided modes in cylindrical waveguides, in which the ratio between the longitudinal and transverse field components only depends to the distance to the interface, here is a very inhomogeneous distribution, extremely different depending on the transverse field component it is considered ( $E_{x}$ or $\left.E_{y}\right)$. Remarkably, there are regions, both inside and outside the waveguide core, in which the longitudinal component is much higher (in absolute value) than the transverse ones. 
This behavior, which could be expected for the $E_{y} / j E_{z}$ case, is quite surprising for the $j E_{z} / E_{x}$ case, since $E_{x}$ is the fundamental component of the guided mode.

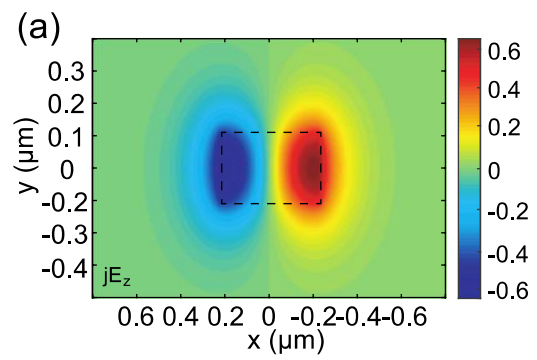

(b)

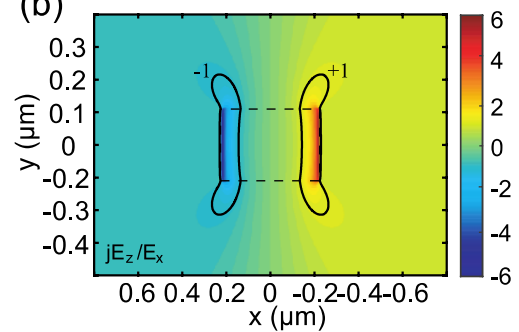

(c)

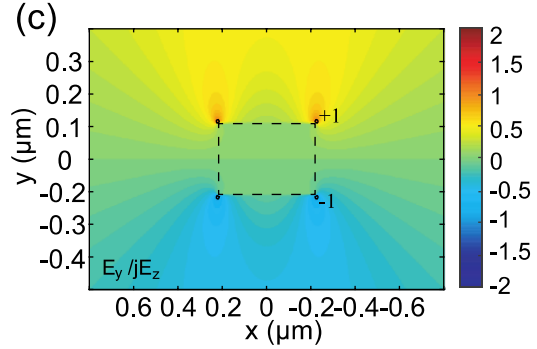

Figure 2.1 Numerically calculated cross-sectional maps of (a) $j E_{z}$; (b) $j E_{z} / E_{x}$; and (c) $E_{y} / j E_{z}$ for the fundamental even mode of a silicon strip waveguide (with $450 \mathrm{~nm} \times 220 \mathrm{~nm}$ cross-section) at $\lambda=1,550$ nm. Contours with $j E_{z} / E_{x}$ or $E y / j E_{z}=1$ and -1, corresponding to points exhibiting local circular polarization of the field projected on the corresponding planes, are also highlighted. The dashed rectangle highlights the interface between the silicon core and the air cladding.

It is also noticeable that maximum values of $\left|j E_{z} / E_{x}\right|$ are obtained mainly inside the waveguide core, this is, within the guided-wave region. Such high values take place in the core regions close to the lateral sidewalls as a result of the reduction of the $E_{x}$ component because of the strong index discontinuity along $x$.

From the electric field components, it is straightforward to obtain the electric contribution of the transverse spin densities of the guided mode as $s_{E}^{x} \propto \operatorname{Im}\left(E_{y}^{*} E_{z}\right)$ and $s_{E}^{y} \propto \operatorname{Im}\left(E_{z}^{*} E_{x}\right)[59,82]$. The cross-sectional maps for both $x$ and $y$ spin densities (in normalized units) of the silicon strip waveguide are depicted in Figure 2.2(a) and (b) respectively. In addition, it can be also obtained the electric contribution to the longitudinal component of the so-called Belinfante's spin momentum 
density (BSMD) [86] as $p_{s, E}^{z} \propto\left(\partial_{x} s_{E}^{y}-\partial_{y} s_{E}^{x}\right)$ [82], which is plotted in Figure 2.2(c).
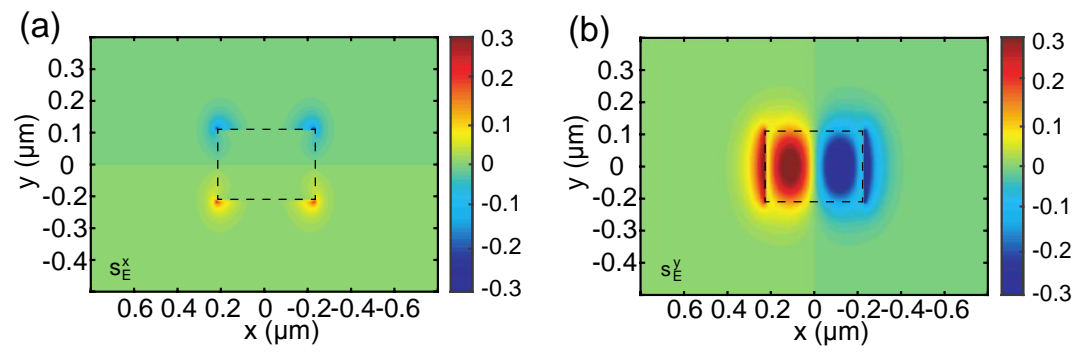

(c)

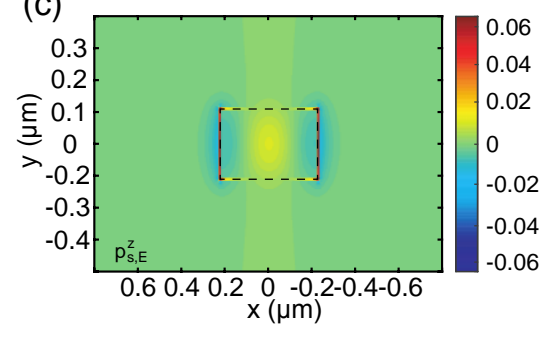

Figure 2.2 Numerically calculated cross-sectional maps of (a) $s_{E}^{x}$, (b) $s_{E}^{y}$, and (c) $p_{S, E}^{z}$ for the fundamental mode of the silicon strip waveguide at $\lambda=1,550 \mathrm{~nm}$.

Notice the strong resemblance between $s_{E}^{x}$ (almost negligible), $s_{E}^{y}$ and $p_{S, E}^{Z}$ along the $x$ axis for an $x$-polarized tightly focused beam (Figure 2.3 in [82]) and the fundamental even in a high-index dielectric waveguide, as a result of the non-negligible longitudinal component of the electric field. However, the strong discontinuities of the field components perpendicular to the boundaries in the guided mode result in some differences, mainly the positive and negative $s_{E}^{x}$ spots near the waveguide corners and the existence of maximum values of $\left|p_{s, E}^{Z}\right|$ at the waveguide boundaries for the guided mode.

In Figure 2.1(b) and (c) it is highlighted contours in which $j E z / E x$ or $E_{y} / j E_{z}$ equals \pm 1 since such regions exhibit local circular polarization on the $x z$ and $y z$ planes (such points are commonly referred to as $\mathrm{C}$ points [87])) associated to the guided even mode propagating along $+z$. This is a clear example of spin-momentum locking, which ultimately results in the SCUE of guided modes: when placing a point dipole emitting circularly polarized waves - or a point scatterer externally illuminated by a circularly polarized wave - at a point in such regions, the even guided mode will be excited towards one direction of the 
waveguide or the opposite depending on the sign of the spin of the excitation wave [70,78].
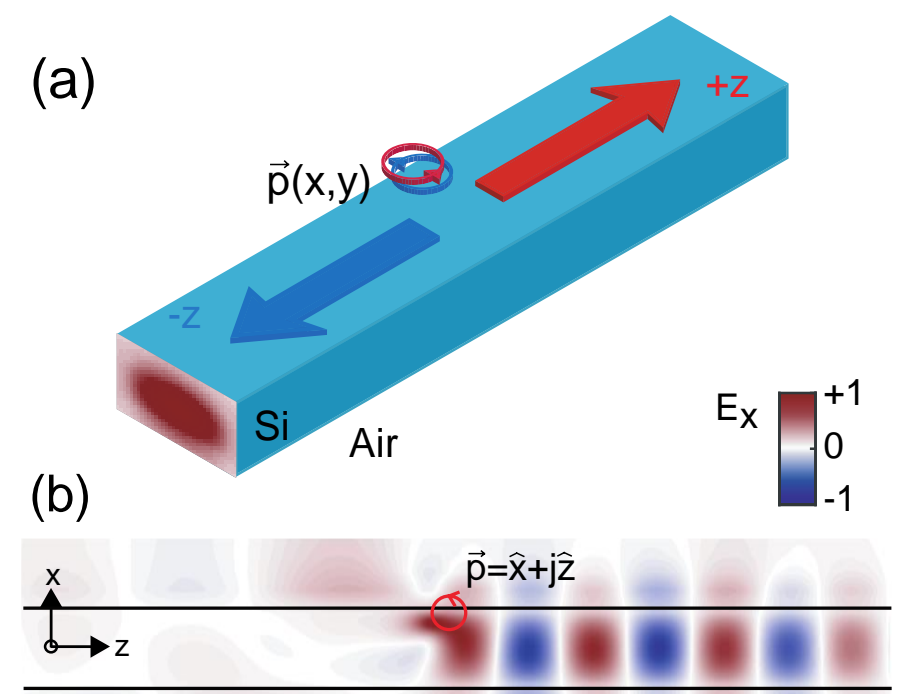

(c)

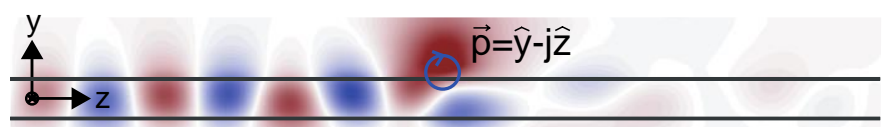

(d)

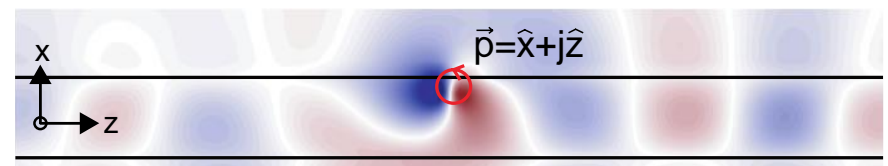

Figure 2.3 a) Scheme of the SCDE of the fundamental even mode in a silicon strip waveguide by using a circularly polarized pointlike dipole with moment $\vec{p}$; Snapshots of the $E_{x}$ component (fundamental TE-like mode) for (b) $\vec{p} \propto \hat{x}+j \hat{z}$ placed at $(x, y)=$ $(0.139,0.13) \mu m(y=0$ cut $)$; and (c) $\vec{p} \propto \hat{y}-j \hat{z}$ placed at $(x, y)=$ $(0.22,0.121) \mu m$ ( $x=0$ cut). (d) Snapshots of $E_{y}$ (TM-like mode) for $\vec{p} \propto \hat{y}+j \hat{z}$ placed at $(x, y)=(0.139,0.13) \mu m(y=0$ cut $)$. 


\subsection{Numerical results}

This idea is illustrated in Figure 2.3(a) and checked via numerical simulations using a full 3D solver (See Methods). The simulation has been performed using a time domain solver and hexahedral mesh with 10 cells per wavelength, except near the dipole where a refinement has been done (reaching 83 thousand cells in total). Open boundary conditions are chosen for all external faces. In Figure 2.3(b) it is shown a snapshot of the propagating $E x$ field on the y $=0$ plane when a pointlike dipole with a dipolar moment $\vec{p} \propto \hat{y}+j \hat{z}$ is introduced at $(x, y)=$ $(0.139,0.13) \mu \mathrm{m}$, where $j E_{z} / E_{x}=1$. Notice that the even mode is clearly excited only towards one of the two possible directions. Such direction could be changed just by switching the spin of the exciting source. A similar result is obtained by placing a circularly polarized dipole $(\vec{p} \propto$ $\hat{y}-j \hat{z})$ at a spatial point in which $E_{y} / j E_{z}=1$, as shown in Figure 2.3(c). Now, the guide mode propagates towards negative $z$ values, as the spin of the source is -1 . This case is especially remarkable since the waveguide mode is unidirectionally excited by an optical source not containing any component along $x$, being $E_{x}$ the main polarization component of the guided mode under consideration. Simulations also show that the power contrast ratio between forward and backward directions is $17 \mathrm{~dB}$ in the first case (b) and $25 \mathrm{~dB}$ in the second case (c), which is a clear signature of unidirectional propagation. Nevertheless, even though very high values of the contrast ratio are achieved, the contrast ratio should be - ideally - infinite. The observation of a noninfinite contrast ratio can be explained by considering that diffraction and reflection of the launched waves in the waveguide boundaries will make the polarization at the dipole position not completely circular [69], resulting in a reduction of the unidirectionality. Results shown in Figure 2.1 and Figure 2.2 describe the transverse spin behavior of the fundamental TE-like mode of a silicon strip waveguide, which is the mode typically employed to build silicon photonics functionalities and devices. Similar results could be obtained for higher-order modes, if the waveguide core area is large enough to support them. These modes will display different profiles for the three electric field components. For instance, here the second guided mode is considered, usually termed TM-like mode and characterized by a main electric field component along the $y$-axis. The field components of this modes will resemble those for the TE-like mode (Figure 2.1 and Figure 2.2) but rotated $90^{\circ}$ around the $z$-axis. Therefore, $E_{x}\left(E_{y}\right)$ for the TM-like mode will look similar to $E_{y}\left(E_{x}\right)$ for the TE-like mode, resulting in completely different conditions to get SCUE of the guide mode. This is checked in Figure 
2.3(d), which is similar to Figure 2.3(b) in what refers to excitation conditions but depict the electric field component along $y$ (and therefore, show the propagation of the TM-like mode).

(a)

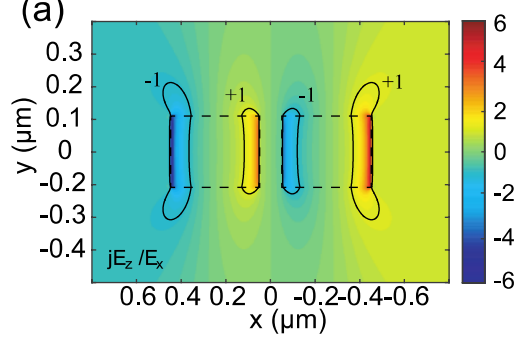

(c)

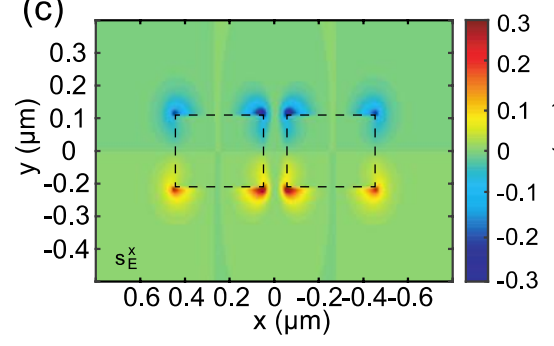

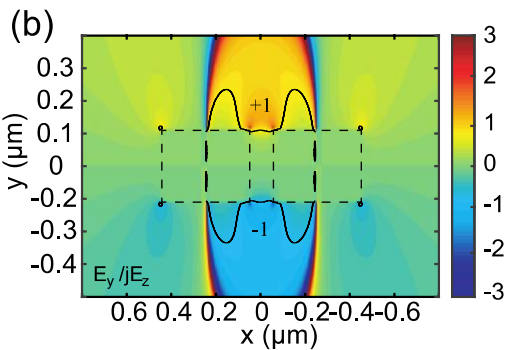

(d)

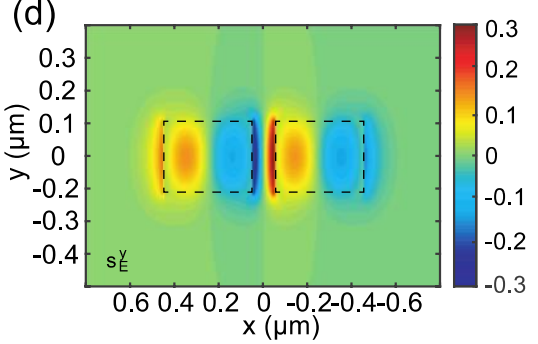

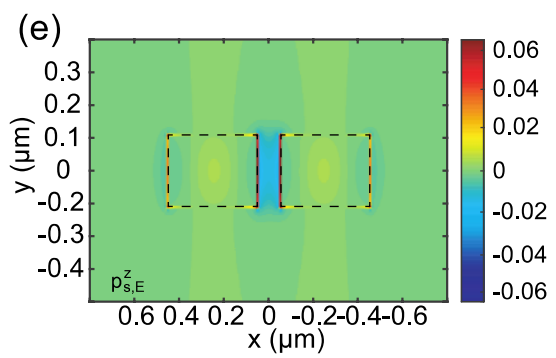

Figure 2.4 Numerically calculated cross-sectional maps of (a) $j E_{z} / E_{x}$, (b) $E_{y} / i E_{z}$, (c) $s_{E}^{x}$, (d) $s_{E}^{y}$, and (e) $p_{s, E}^{z}$ for the fundamental mode of the silicon slot waveguide (comprising two strip waveguides with $300 \mathrm{~nm} \times 220 \mathrm{~nm}$ cross-section spaced by a 100 $\mathrm{nm}$ gap) at $\lambda=1,550 \mathrm{~nm}$.

It can be seen that in these cases the TM-like mode is not unidirectionally excited because the local polarization of the evanescent tails at the point where the dipole is placed is not completely circular but elliptical. This proves that spin-orbit coupling in multimode waveguides depends on the mode that is excited, which has to be taken 
into account when using this effect to build nano-optical devices aimed at polarization manipulation.

Similar results can also be obtained for another kind of waveguide commonly employed in silicon photonics: the slot waveguide [86]. The slot waveguide comprises two parallel strip waveguides with a small gap between them.
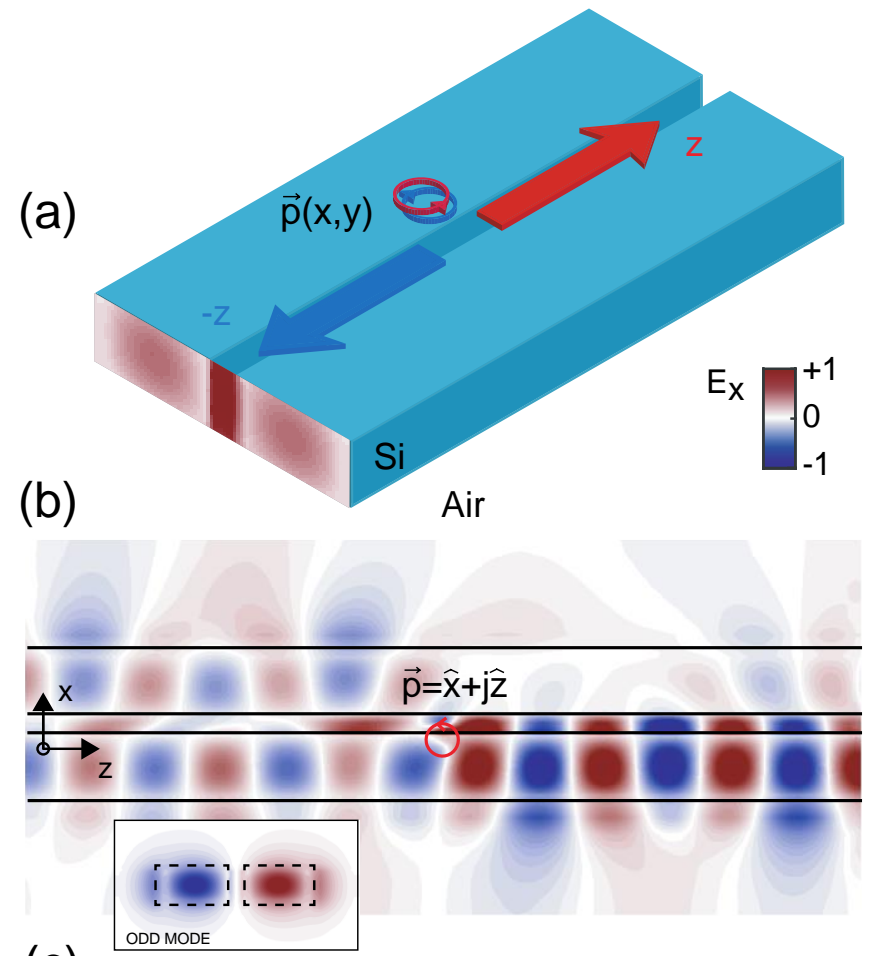

(c)

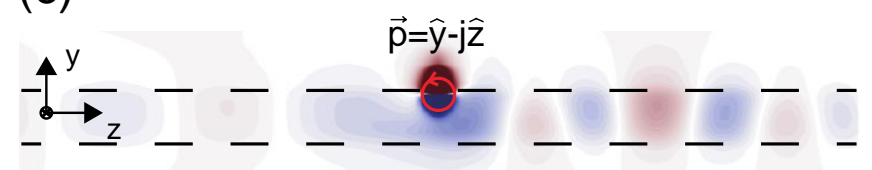

Figure 2.5 (a) Scheme of the SCDE of the fundamental even mode in a silicon slot waveguide by using a circularly polarized pointlike dipole with moment $\vec{p}$; Snapshots of the $E_{x}$ component for (b) $\vec{p} \propto \hat{x}+j \hat{z}$ placed at $(x, y)=(-0.059,0.115 \mu \mathrm{m})(y=0$ cut $)$ and (c) $\vec{p} \propto \hat{y}+j \hat{z}$ placed at $(x, y)=(0.001,0.101) \mu \mathrm{m}(x=0$ cut $)$. 
Remarkably, if the gap is sufficiently small, the fundamental even mode exhibits a strong $E_{x}$ confinement in the slot region, making this waveguide well suited for sensing [88] and all-optical processing $[25,26]$. Therefore, it makes sense to find out if such waveguide also present useful properties in what refers to the transverse spin of its fundamental mode, especially in the active slot region. Figure 2.4 shows the simulation results obtained for a slot waveguide. Since the slot waveguide can be considered as two coupled strip waveguides, many of the observations on polarization properties previously done for the strip case can also be applied here, mainly the existence of very high values of the transverse spin densities. The SCUE of the slot mode is demonstrated in Figure 2.5 by using both $x z$ and $y z$ point-like circular dipoles. In both cases, the power ratio between forward and backward directions higher than $10 \mathrm{~dB}$.

Again, this is a signature of unidirectional propagation, but the value is not infinite because of the existence of diffracted and reflected waves that modify the polarization at the position of the excitation source. Remarkably, a guided mode with odd symmetry with respect to the $x=$ 0 plane (see the $E_{x}$ field pattern in the inset) propagating along negative $z$ values is observed. This mode also arises from the splitting of the fundamental even mode of the isolated strip waveguide as a consequence of the strong coupling between parallel waveguides. As for the case of the strip waveguide, here it is observed that the condition for SCUE of the fundamental even mode only hold for that mode, and a higher-order mode is also excited without unidirectional behavior.

So, in this chapter it has been demonstrated the transverse spin as well as the orbit-coupling features in silicon strip and slot waveguides. All these aspects should be taken into account in the following chapters. In Chapter 3 and Chapter 4 silicon waveguides will be used to excite nanoantennas embedded in them through a gap. For the correct feeding of the nanoparticle the guided mode configuration seen in this chapter must be taken into account. However, in Chapter 5, the areas of the guided mode that exhibit spin-momentum locking producing the SCUE of guided waves will be used for polarization analysis applications. 


\section{Chapter 3 \\ Embedding plasmonic nanoantennas in silicon waveguides}

In this chapter, numerical simulations and experiments of the optical response of gold nanostrip antenna embedded in a silicon strip waveguide gap at telecom wavelengths are reported. It is shown that the spectral features in transmission and reflection when the metallic nanostructure is inserted in the gap are extremely different to those observed in free-space excitation. It is demonstrated that interference between the guided field and the electric dipolar resonance of the metallic nanostructure results in high-contrast (> 10) spectral features showing an asymmetric Fano spectral profile. Secondly, it is revealed a crossing in the transmission and reflection responses close to the nanostructure resonance wavelength as a key feature of the presented system.

\subsection{Introduction}

Metallic nanostructures supporting localized surface plasmons have become key elements for manipulating light fields at the nanoscale $[2,89,90]$. Their ability for extreme concentration of the optical field in deep subwavelength volumes, which naturally boost any kind of optical interaction, has been employed to implement highly sensitive miniaturized biosensors [91] or to demonstrate enhanced nonlinear behavior [92] amongst other applications. Owing to its ultrasmall size, experimental characterization of individual nanostructures supporting plasmonic resonances is far from trivial because of the diffraction limit [93]. As a result, arrays of non-interacting metallic nanostructures created on planar substrates have been usually employed for optical characterization [94]. When measuring such arrays, several elements are simultaneously illuminated at a time, and the response of an isolated element is approximated by the averaged response of the illuminated structures, with the assumption that all of them are identical. This precludes to properly identify the performance of a single structure, with all the physical richness that it can provide. Scanning individual nanostructures requires strong focusing of the incident light $[11,12]$. This approach, however, besides being inefficient because light focusing is diffraction-limited and the nanostructures exhibit deep 
subwavelength cross-sections, is restricted to the measurement of a single element at a time, making unpractical the simultaneous measurement of multiple plasmonic elements built on a substrate. This limitation may become a roadblock in many applications, remarkably biosensing, which would ultimately require scanning multiple nanostructures in parallel and in real-time in order to get fully profit of the miniaturization provided by plasmonics.

Exciting the metallic nanoparticles via dielectric waveguides is an appropriate way to solve this drawback: a single waveguide could both illuminate an individual nanostructure placed close to it and collect its response (or at least, part of it), enabling to measure its properties in a single shot. By using multiple waveguides simultaneously fed by a single optical source (for instance, using passive $1 \times N$ power dividers), a number of metallic nanostructures could be simultaneously illuminated and their response measured in real time at the waveguides output. As mentioned above, unlike metallic waveguides, dielectric waveguides can become practically lossless, enabling transfer of optical information between distant parts of a photonic chip at the centimeter scale and beyond. Owing to the ultrasmall dimensions of the metallic nanostructures under consideration, high-index waveguides enabling strong field concentration in transverse dimensions around $\lambda / 2 n$ (being $\lambda$ the free space wavelength and $n$ the waveguide core index) would be preferred. Amongst the available technological platforms for high-index integrated optics, silicon photonics presents several advantages, mainly an easy fabrication using standard microelectronics tools, ultimately enabling low-cost production at large-volumes [95,96,97]. In addition, whilst silicon can be used as a guiding element for near-infrared devices, silicon nitride waveguides enabling visible light guidance can be also produced in the same technological platform [98-99].

Recent experiments have demonstrated the excitation of subwavelength-size plasmonic nanostructures placed on top of silicon and silicon nitride waveguides at visible and infrared wavelengths $[46,55,56,100,101,102,103]$. The coupling between the guided field and the nanostructure takes place in the evanescent region of the waveguide, which is excited by the fundamental TE-like mode having the fundamental electric field component parallel to the chip plane (see Figure 3.1(a)). This results in relatively small interaction efficiencies (typically $<10 \%$ although $\sim 19 \%$ is reported in [102]) so in many occasions a number of nanostructures need to be placed on the waveguide (see Figure 3.1(b)) in order to produce observable effects (a 
dip in transmission) at the waveguide output $[56,101,102]$. Such weak coupling produces low power contrast $(<2)$ between maxima and minima in the measured spectra at the waveguide output, preventing its use in applications such as biosensing or all-optical switching which require high contrast to differentiate states. To overcome this issue and fully excite the nanostructure, it would be convenient to embed the nanostructure within a gap created in the waveguide (see Figure 3.1(c)), as demonstrated numerically and experimentally in this chapter at telecom wavelengths.

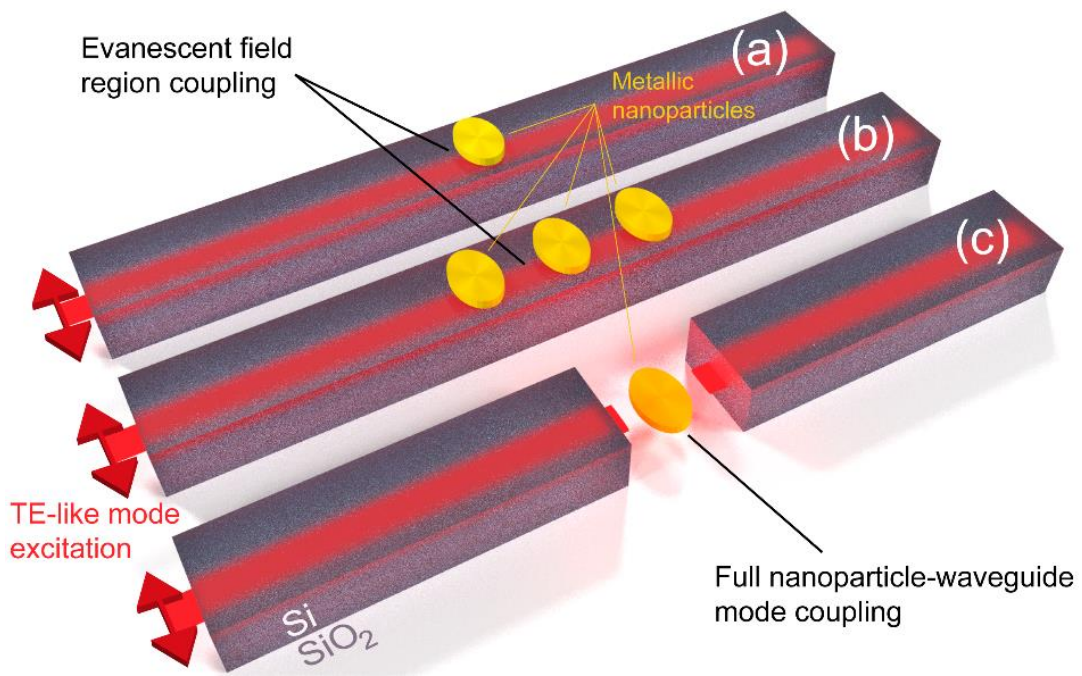

Figure 3.1 Excitation of subwavelength-size plasmonic nanostructures. (a) The nanoparticle is placed in the evanescent region of the waveguide mode. (b) Improving the interaction efficiency using several nanoparticles in the evanescent region of the waveguide mode. (c) Full excitation of the nanoparticle aligning it with the waveguide optical axis.

\subsection{Description of the concept}

The concept detailed in this chapter is schematically described in Figure 3.2. A very small gap $(g)$ separates two silicon waveguides (silicon nitride would provide a similar performance) with rectangular crosssection, being the plasmonic nanostructure placed in the middle of it and centered at the waveguide axis. This way, a maximum interaction 
of the propagating light field with the nanostructure is ensured, mainly in comparison with the approach in which the nanostructure is placed on top of the waveguide. As shown below, reflection at the gap boundaries can be relatively small. When illuminated, part of the light scattered by the nanostructure will be emitted towards the input port (backscattering) or towards the output port (forward scattering), interfering with the guided reflected and transmitted fields that have not interacted with the nanostructure, respectively. In addition, the metallic nanostructure will both scatter light out of the waveguides (out-of-plane but also in-plane) and absorb part of the incoming power, especially when a localized surface plasmon resonance (LSPR) is excited.

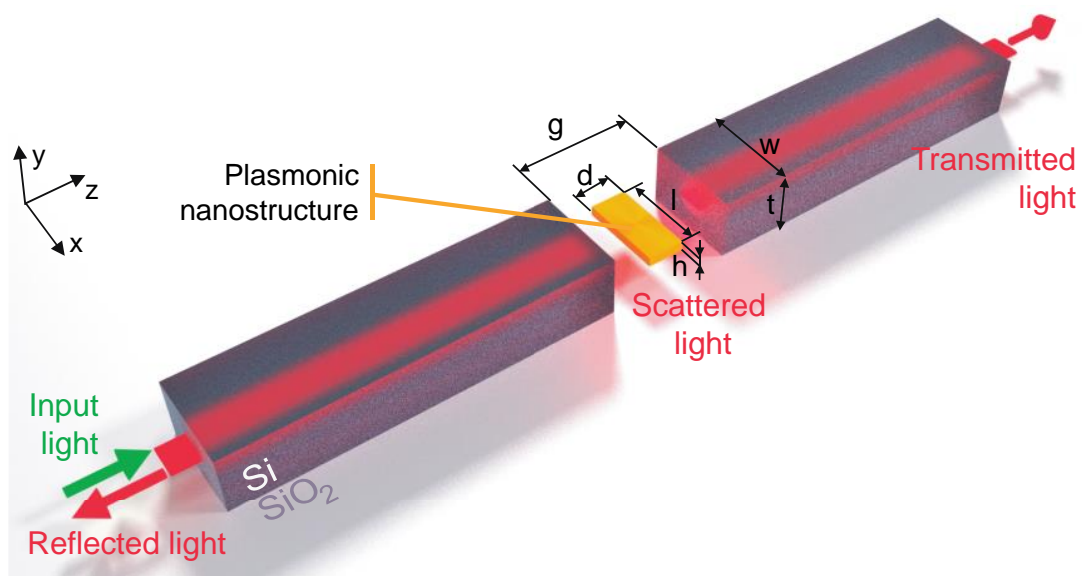

Figure 3.2 Scheme of the proposed approach for full excitation of a plasmonic nanostructure (in this chapter as well as in the figure, it is considered a gold nanostrip with dimensions $l \times d \times h$ placed inside a sub-micron gap (width $g$ ) created in a strip silicon waveguide with rectangular cross-section $w \times t$. The surrounding medium is silica, although other low-index dielectric (such as air) could be used.

Therefore, by measuring the optical power at both ports (transmission and reflection) it is possible to get an accurate estimation of the absorbed and out-of-guide scattered power of the LSPR. Notice the resemblance of this approach with that studied by R. Sapienza and colleagues [62] where a metallic nanoantenna is embedded within the silicon waveguide core, which is drilled by a hole with the nanoantenna dimensions. Although their approach enables a full excitation of the 
nanoantennas when perfectly aligned with the waveguide optical axis, it is limited in what refers to the fabrication (high-aspect ratio etching of silicon) as well as the type of metallic nanostructure to be inserted (in the approach presented here, any kind of plasmonic nanostructure could be introduced, including elements with complex shapes supporting magnetic [104] or Fano resonances [105] or exhibiting directional scattering $[63,64])$.

\subsection{Numerical results}

For the numerical study of this approach the strip waveguides have been considered, with dimensions $w=400 \mathrm{~nm}$ and $t=250 \mathrm{~nm}$, made of crystalline silicon and are surrounded by silica.

Before analyzing the complete structure, the waveguide without any nanoparticle at the gap is considered in order to know about the influence of the gap into the transmission and reflection behavior of the waveguide. Figure 3.3 shows the transmission $T$ and reflection $R$ spectra for the TE-like mode (a) and TM-like mode (b) for four different gap lengths in the wavelength window between 1.1 and 1.9 $\mu \mathrm{m}$. To account for the waveguide spectral response, results in Figure 3.3 are normalized with respect to the transmission spectra of the isolated waveguide (without gap). It is found that the shorter the gap is, the higher the transmission and the lower reflection will be, as could be expected by considering the light exiting the input waveguide will diverge because of the strong confinement and scattering will grow as the gap width increases. This also results in a reduction of the scattering losses for larger wavelengths. Notice that the gap can be considered to operate as a Fabry-Perot cavity with large radiative losses (light that is scattered out of the waveguides) and subwavelength length, which precludes the observation of ripples in the transmission spectra. 
(a)

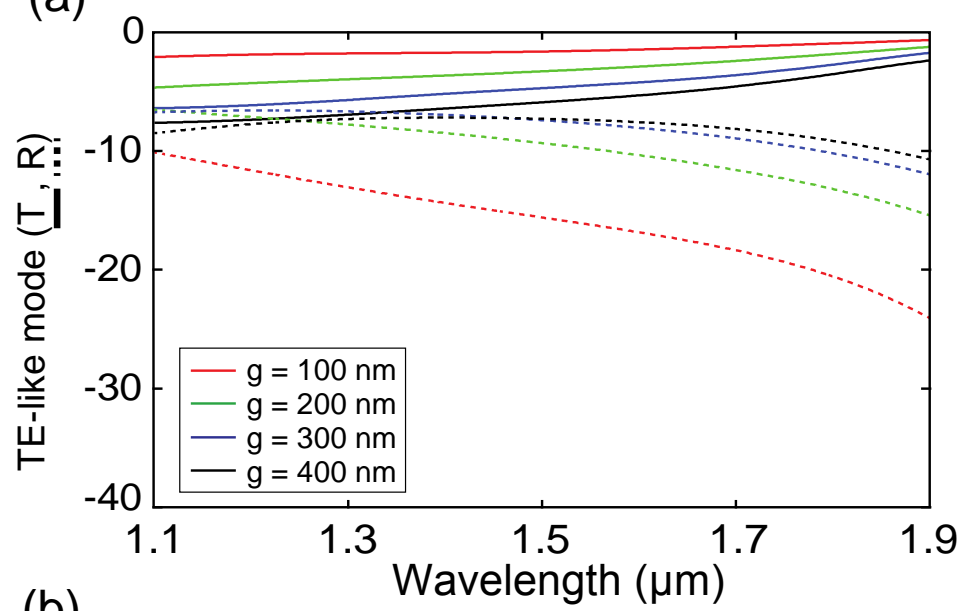

(b)

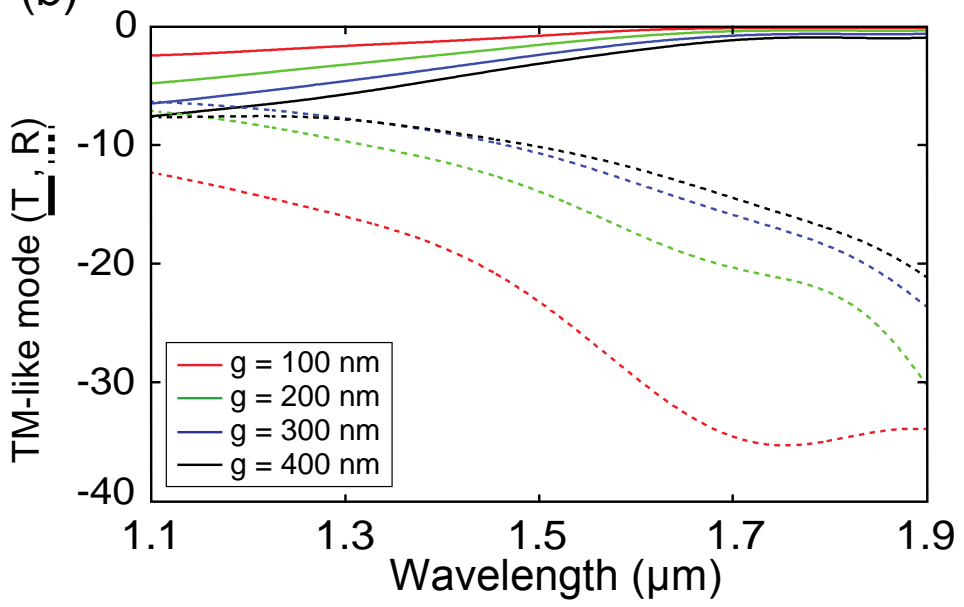

Figure 3.3 Numerical study of the gap effects. Normalized transmission (T) and reflection (R) spectra for excitation using the fundamental TE-like (a) and TM-like (b) modes at different gap widths. The vertical axis is in $\mathrm{dB}$ units.

Here is also calculated the electric field profile in the middle of a 300 $\mathrm{nm}$ gap for both TE and TM-like modes. Results are shown in Figure 3.4. It can be seen that, besides a large field component corresponding to the fundamental component of each guided mode ( $E_{\mathrm{x}}$ for the TE-like mode and $E_{\mathrm{y}}$ for the TM-like mode) there is also a large longitudinal component $E_{\mathrm{z}}$ with an asymmetric profile in both cases. 

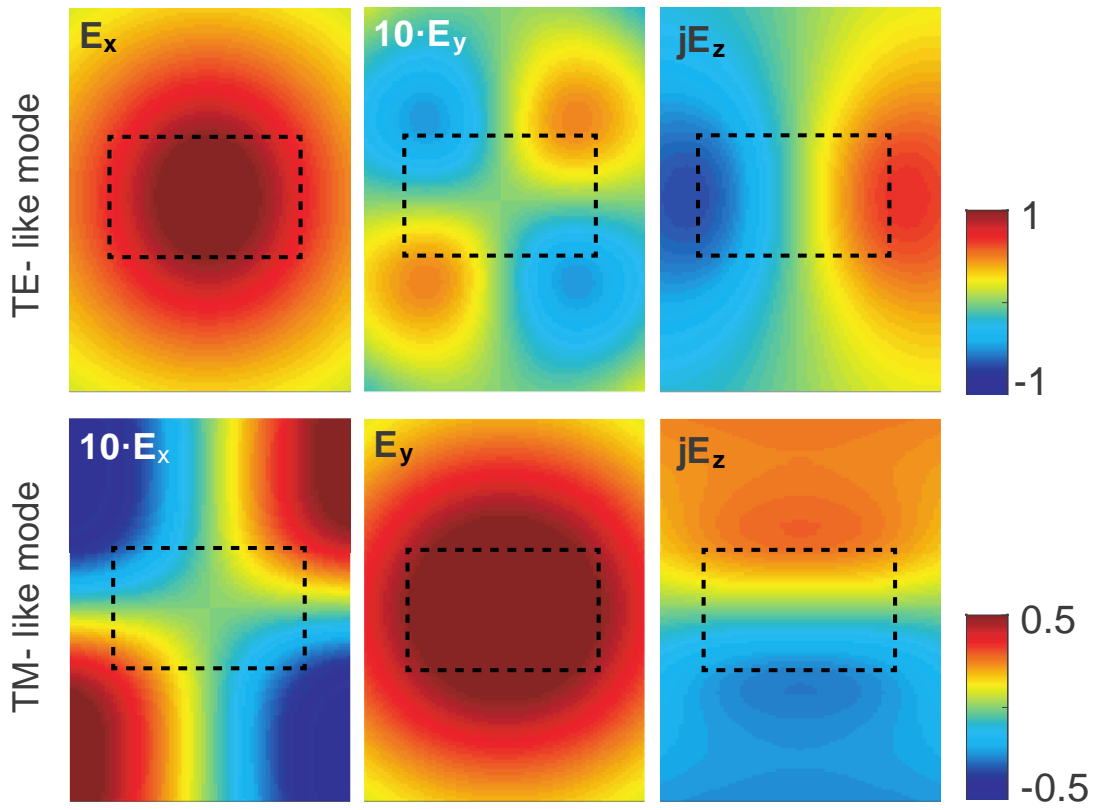

Figure 3.4 Snapshot of the electric field components recorded at a plane placed at the middle of the gap $(\mathrm{g}=300 \mathrm{~nm})$ for TE-like and TM-like waveguide modes.

This is related to the existence of a large transverse spin in silicon waveguides as a result of the strong confinement and could be used for polarization manipulation when placing subwavelength nanoantennas in the proximity of the waveguide [70,77]. In the case presented in this chapter, the existence of large longitudinal fields in the gap makes a strong difference in comparison with typical excitation of plasmonic nanostructures using free-space plane-wave-like light where the transverse component is dominant.

Such longitudinal components must be carefully considered when trying to excite electric dipolar resonances (as, for example, in the case that is considered in this chapter) with the transversal field, mainly for large nanostructures or nanostructures placed out of the optical axis. But besides this, it can be also considered the existence of such components as an interesting opportunity since it offers the possibility to play with the gap fields and the nanostructure shape and position to excite complex LSPRs that could result in effects not achievable with free-space excitations such as, for instance, directional scattering $[63,64]$. 
After studying the response of the waveguide with gap, it is time to consider the response of the metallic element to be introduced in the gap when isolated. For simplicity, it is considered a basic plasmonic nanostructure: a gold nanostrip with rectangular cross-section $(h \times d)$ and length $l$ whose dimensions can be chosen to exhibit an electric dipole LSPR at telecom wavelengths for excitation with an $x$-polarized incoming wave. Figure 3.5 shows the calculated scattering cross section (SCS) and absorption cross section (ACS) for a nanostrip with dimensions $l=320 \mathrm{~nm}, d=155 \mathrm{~nm}$ and $h=40 \mathrm{~nm}$ surrounded by silica. The LSPR wavelength is about $1.75 \mu \mathrm{m}$ though it can be easily tuned by modifying $l$. Note that the ACS value is multiplied by 5 to show more clearly that the maximum of the absorption is not located at the same wavelength as the maximum of the scattering, as expected.

Finally, the full system is considered: the metallic nanostrip placed within the gap as shown in Figure 3.2. To achieve a maximum interaction the nanostrip is aligned with the optical axis of the waveguide. In order to excite the LSPR, the TE-like mode of the silicon waveguide has to be launched so that a large $E_{\mathrm{x}}$ component impinges on the nanostrip according to the results in Figure 3.4. Notice that the longitudinal field component (see $E_{\mathrm{z}}$ panel in Figure 3.4) will also have some effects on the metallic nanoparticle because of its length (320 $\mathrm{nm}$ ), which does not take place in the SCS and ACS calculations where the nanoparticle is isolated and the incident field is purely $x$-polarized. When excited, the radiation emitted by the currents circulating on the nanostrip (here completely acting as a nanoantenna) will interfere with the transmitted and reflected guided waves, which will modify the responses shown in Figure 3.3. Part of the emitted radiation will not be captured by the waveguides so will be lost, contributing to the scattering loss arising from the light coupling from waveguide to waveguide. Finally, there will be also a strong absorption, proportional to the ACS of the isolated nanostrip, as a result of the ohmic loss of the metallic nanostructure. Figure 3.5 also shows the transmission and reflection of the gold nanostrip embedded in a $g=300 \mathrm{~nm}$ gap. Counterintuitively, there is not a minimum in transmission associated with the LSPR peak wavelength, as it happens when the metallic scatterer is embedded into the silicon waveguide [62]. A crossing of the transmission and reflection curves is observed instead in the region of the LSPR wavelength. Such a crossing, which will be studied in depth in the next chapter (section 4.2.1), can be considered as a signature of the excitation of the LSPR in this system. At wavelengths below the LSPR, there is a large reflection and low transmission, being this situation reversed at wavelengths above the LSPR. This behavior can 
be explained by considering that the phase of the radiation emitted by the metallic nanostrip, which can be considered as a half-wavelength electric dipole antenna, changes with wavelength as expected for a polarizable particle, being the interference with the guided field constructive (destructive) for reflection (transmission) at wavelengths below the LSPR and vice versa for wavelengths above the LSPR.

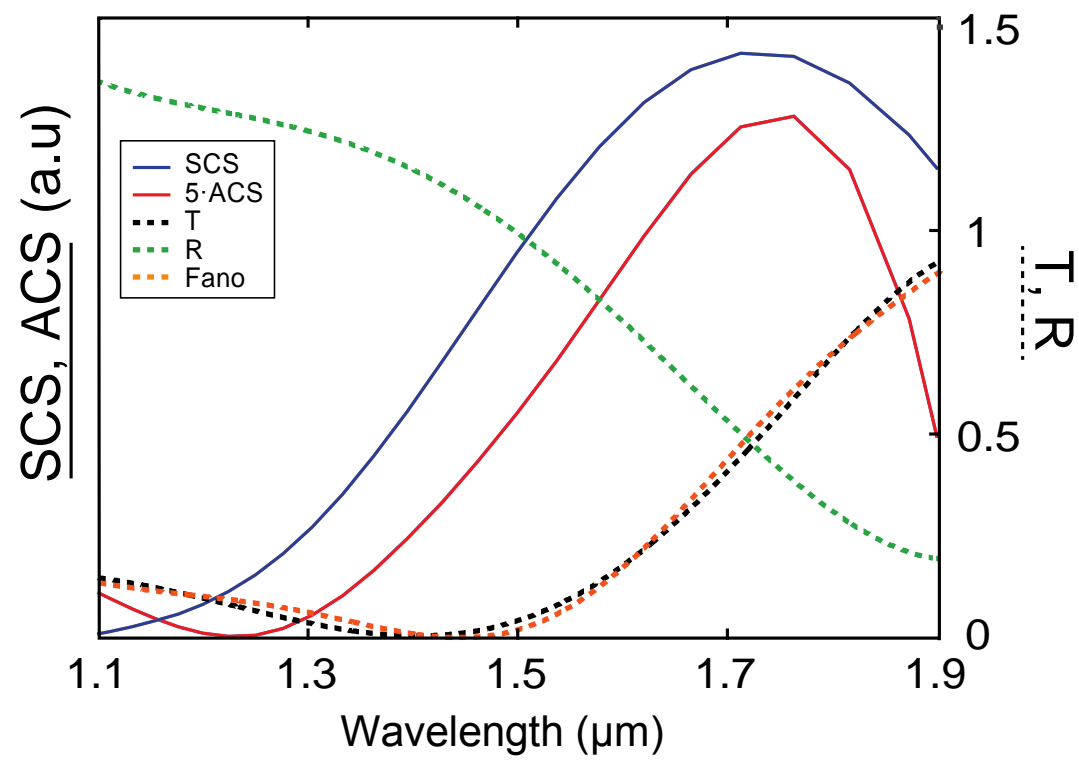

Figure 3.5 Numerical results when the nanostrip is included. Simulated scattering (RCS, blue solid), and absorption (ACS, red solid) cross-sections for a gold nanostrip with dimensions $l=320$ $\mathrm{nm}, d=155 \mathrm{~nm}$ and $h=40 \mathrm{~nm}$, surrounded by silica. Transmission ( $T$, black dashed) and reflection ( $R$, green dashed) of the complete system with the nanostrip embedded in a $g=300 \mathrm{~nm}$ gap and Fano fitting (orange dashed) to the simulated transmission.

Remarkably, destructive interference in the transmitted signal reaches its maximum in the wavelength region around $1.4 \mu \mathrm{m}$, where the transmittance goes close to zero. In this region, the interfering paths (the field transmitted from waveguide to waveguide and the field radiated by the nanostructure) will exhibit similar amplitudes but a $\pi$ phase shift between them. This confirms that the plasmonic nanostructure is fully excited by the incoming field, in contrast to the particle-on-top approach, for which the amplitude of the radiated field will be much smaller than that of the guided wave. In addition, it is found that the reflection curve is in good agreement with the numerical 
results in [106] for a metallic nanoantenna placed at the output of a dielectric waveguide.

The asymmetry of the transmission spectrum profile can be well explained in the context of Fano resonances [107,108]. In this case, the optical signal propagating from waveguide to waveguide across the gaps plays the role of the broad continuum whilst the first-order LSPR of the gold nanostrip acts as discrete (although spectrally broad) resonance [109]. The transmission response can be fitted to a Fano resonance with $F=0.55, \omega_{0}=11.23 \cdot 10^{14} \mathrm{rad} / \mathrm{s}$ and $\gamma=470 \mathrm{~nm}$, where $F$ describes the degree of asymmetry, whilst $\omega_{0}$ and $\gamma$ correspond to the position and width of the isolated nanoparticle LSPR respectively [107]. The resulting curve is depicted in Figure 3.5, showing a good agreement with the response obtained from numerical simulations.

Moreover, it is possible to spectrally shift the transmission and reflection responses by changing the dimensions of the nanostrip. Results obtained when varying the dimensions $l$ and $d$ ( $h$ is kept equal to $40 \mathrm{~nm}$ ) for TE-like excitation are shown in Figure 3.6(a) and (b), respectively. The curves are normalized with respect to the results without the nanostrip, this is, only considering the effects of the $300 \mathrm{~nm}$ gap. Regardless of the dimensions, it is observed that in all the cases the crossing point between the transmission and reflection curves. Remarkably, the normalized transmission is above $0 \mathrm{~dB}$ in the long wavelength region. This means that the scattering introduced by the gap is reduced when the metallic nanoantenna is inserted: a portion of the scattered field is captured by the nanoantenna and radiated towards the output waveguide, where it interferes constructively with the guided field producing an increase in the total transmission. When $l$ increases, a red-shift of both the transmission dip and the transmission-reflection crossing point is observed. This is a direct consequence of the nanostrip behaving as a half-wave dipole nanoantenna. In addition, it can be seen that the transmission minimum is also modified when changing the nanoantenna dimensions. Indeed, in Figure 3.6(b) is observed that whilst a modification in $d$ does not produce appreciable changes in the transmission dip wavelength, it strongly modifies its depth, enabling values of the order of $-50 \mathrm{~dB}$, which is quite impressive if it is considered that the scatterer dimensions are subwavelength. 


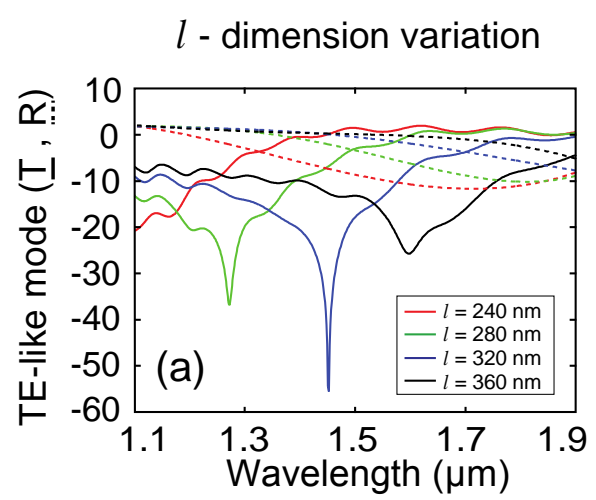

$d$ - dimension variation
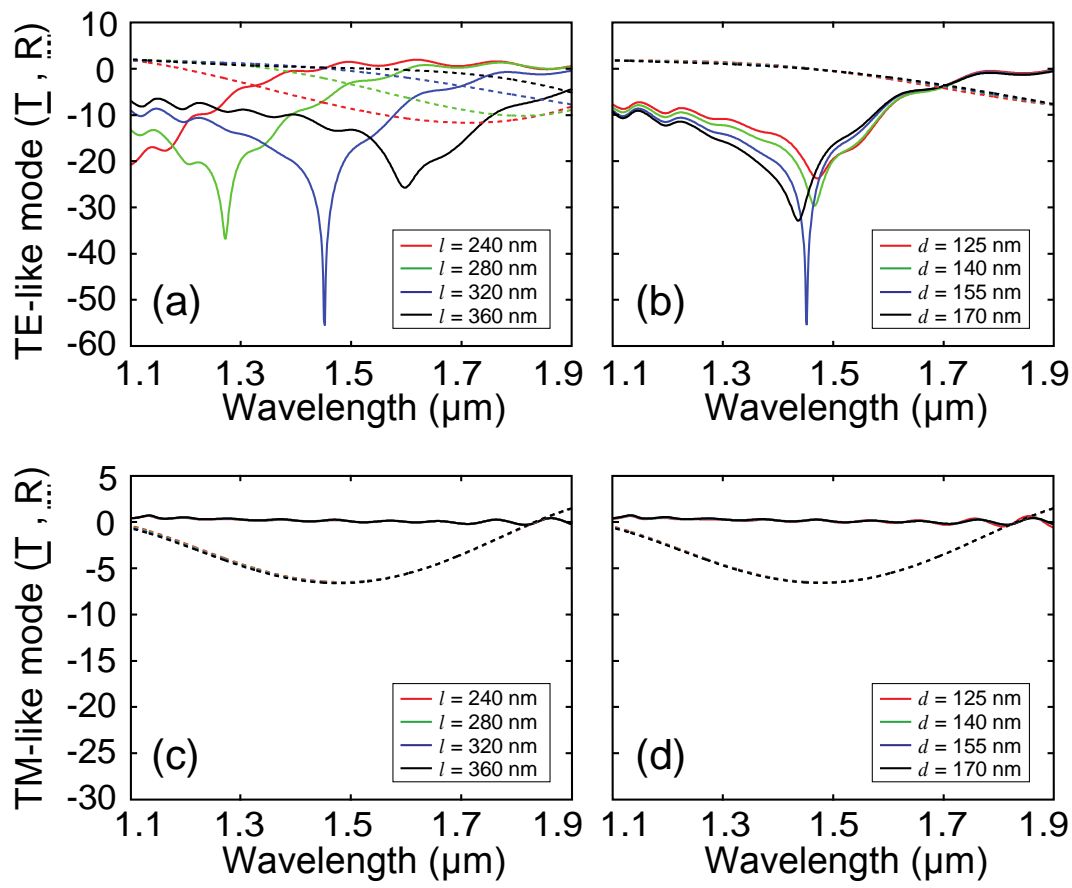

Figure 3.6 Transmission and reflection through the silicon waveguide at TE-like (top) and TM-like (bottom) excitation for variations of the nanostrip dimensions (l and $\mathrm{d}$ ). Both transmission (solid line) and reflection (dashed line) are represented in $\mathrm{dB}$.

This available contrast in the transmission spectrum $(>50 \mathrm{~dB}$ or five orders of magnitude) extremely exceeds the values achieved when illuminating metallic nanostructures placed on a substrate in an out-ofplane configuration or in the configuration when the nanostructure is placed on top of the waveguide, where the amplitude contrast in transmission (or reflection) is within one order of magnitude. The availability of such large contrast in the transmission levels may enable disruptive performance in several applications that make used of LSPRs of isolated nanostructure. For example, it would enable to get an ultralarge dynamic range for detecting substances if employed in biosensing. If utilized for switching purposes, it could allow for ONOFF contrasts or modulation depths well above $10 \mathrm{~dB}$, which should be sufficient to differentiate bits at the switch output.

Results for TM-like excitation are depicted in Figure 3.6(c) and (d) for variations in $l$ and $d$ respectively. In this case, the LSPR is not excited 
because the electric field in the center of gap region has fundamentally a component along the y-axis and there are no appreciable features in the obtained spectra regardless of the nanostrip dimensions.

\subsection{Experimental results}

Figure 3.7 shows scanning-electron-microscope (SEM) images of several fabricated samples. It can be seen that some deviations arisen in the fabricated samples, mainly in the position of the nanostructure within the gap. This could potentially produce different results when measuring the system in opposite directions. To account for this, it has been characterized the fabricated structure in both transmission directions (from left to right and vice versa) and also for both guided modes (TE-like and TM-like), giving a set of four possible measurements. Figure 3.7 also describes the colour code employed to describe the experimental measurements reported below. The samples were characterized by using a standard butt-coupling system typically used to measure transmission spectra in photonic chips. As a light source, a tunable laser covering the range between 1260 and $1630 \mathrm{~nm}$ is used. A polarization controller was used to select the mode propagating along the silicon waveguides. A lensed fiber coupled the light to the sample, in a process with coupling losses of the order of 10 $\mathrm{dB}$. At the output of the sample, there is an objective, a polarization filter and a splitter, which was used to split the light beam into two orthogonal paths: one directed to a near infrared camera to localize the output light spot and the other one directed to a power meter (Newport 1930C). The lensed fiber and the sample were placed on nanopositioners (not shown in the figure for the sake of clarity) which allow for aligning the input fibre with each waveguide included in the sample.

In order to compare the transmission spectra obtained in simulations and measurements, numerical calculations using the real geometrical data of the fabricated samples have been performed, including alignment deviations (see Figure 3.7). The resulting transmission spectra once normalized with respect to the system without nanoparticle are depicted in Figure 3.8. Since the range of wavelengths available for characterization is smaller than those shown in Figure 3.5, there is not access to all the details arising from the numerical simulations. 

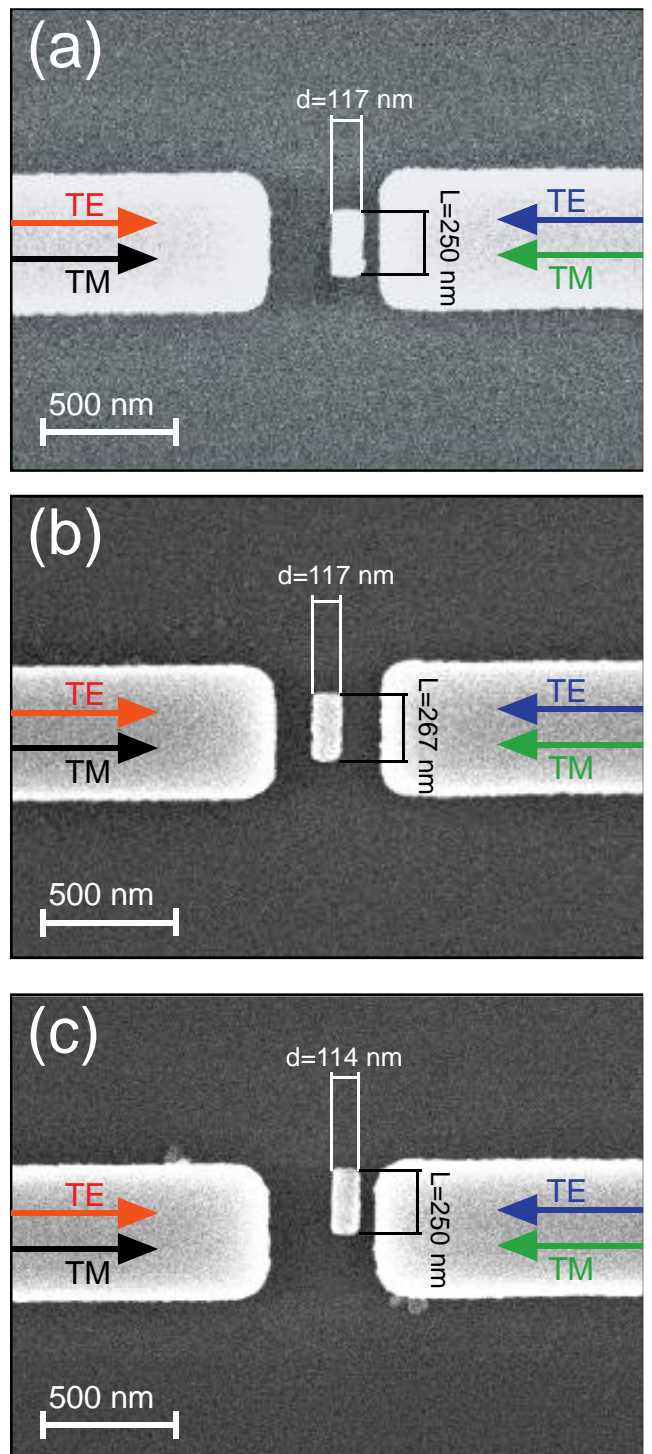

Figure 3.7 SEM images of three fabricated structures. The top view of the fabricated systems is shown, with the top dimensions of the gold nanostrip (at the center) depicted in nm. The thickness of the nanostrip is $40 \mathrm{~nm}$. Deviations in the nanoparticle position or dimensions were taken in account for the simulations.

Still, it is possible to measure a relevant portion of the spectrum and, more importantly, it can be observed the most interesting features of the structure for TE-like excitation: low transmission in the short 
wavelength region and transmission above $0 \mathrm{~dB}$ in the long wavelength region, with a $0 \mathrm{~dB}$ crossing close to the LSPR wavelength. For TMlike excitation, the transmission oscillates around $0 \mathrm{~dB}$, as expected from the fact that the LSPR is not excited so the transmission with and without nanostrips remains almost the same. Notice that the experimental results match properly with the performed simulations.
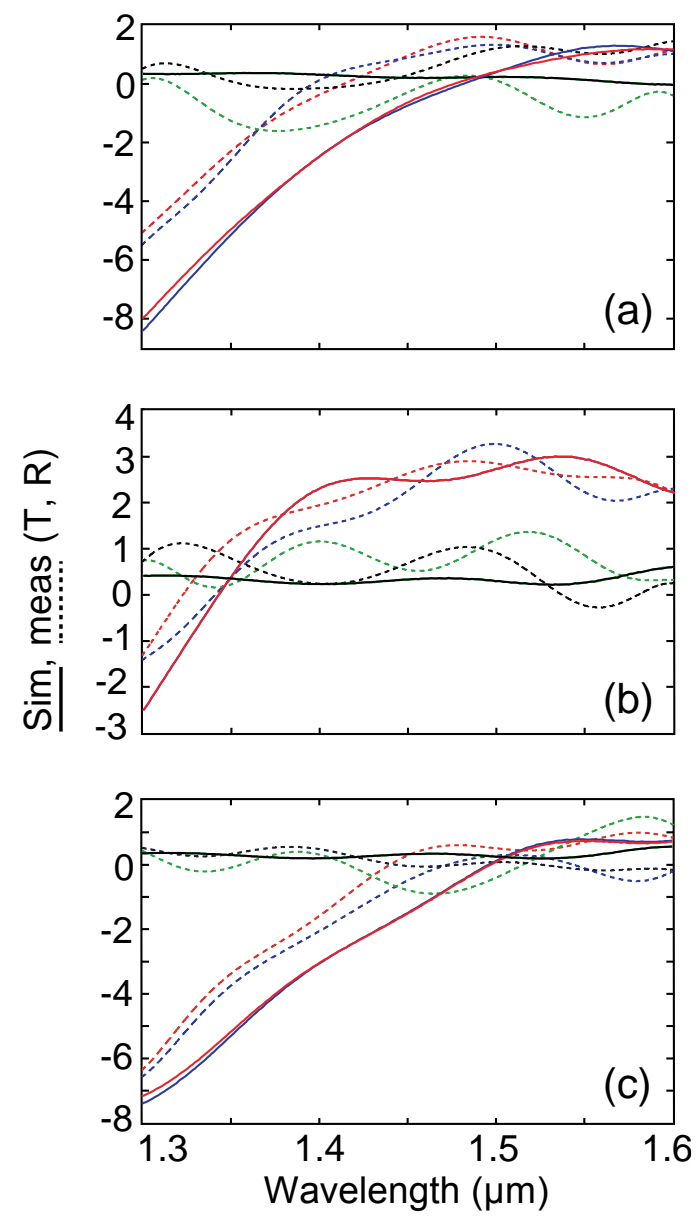

Figure 3.8 Simulated and measured transmission $(T$, in $\mathrm{dB})$ with respect to a waveguide with gap but without nanostrip. Experimental results are depicted in dashed line, while simulated results are depicted in solid line. TE-like and TM-like transmission from left to right (right to left) are depicted in red and black (blue and green), respectively. Each subfigure $(a, b, c)$ corresponds to the structures shown in Figure 3.7 (a,b,c) respectively. 
Remarkably, there are not huge difference between transmission results along counter-propagating paths even for the cases of significant misalignments. This can be explained by considering that the excitation of the LSPR is quite efficient in all the cases and the shift in the position of the nanotrips with respect to the ideal case are not sufficient to produce phase shifts that could result in observable changes in the transmission.

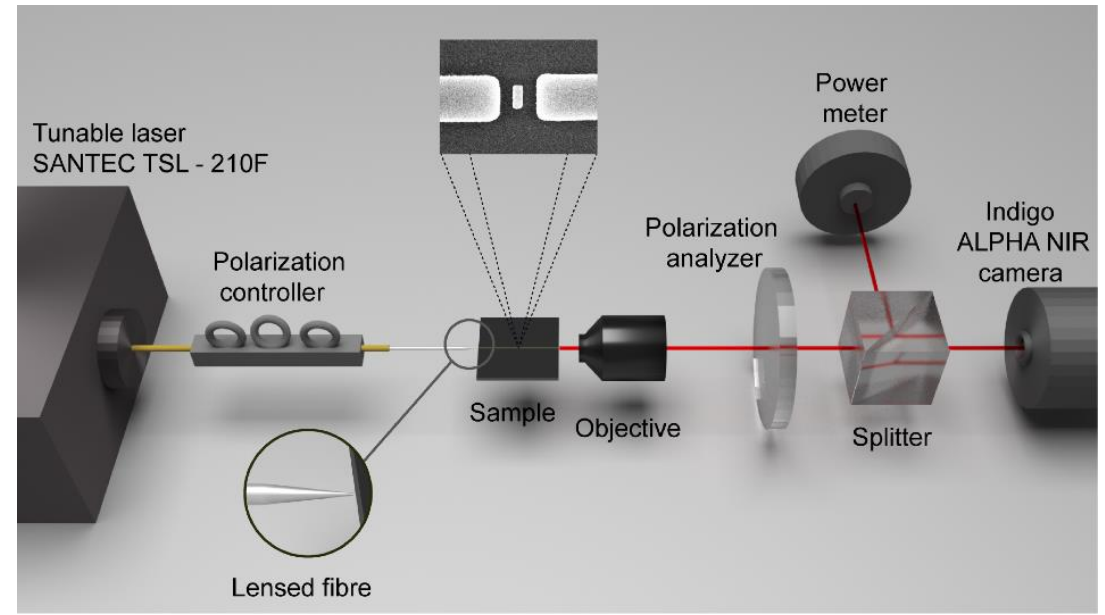

Figure 3.9 Scheme of the optical set-up used in the experimental measurements. Insets show details of the lensed fiber and a SEM of the gap region of one of the tested samples.

Figure 3.8 shows the measured contrasts between transmission maxima and minima of the order of $10 \mathrm{~dB}$. However, the fabricated structures show even larger contrast which are not shown in the transmission spectra because the minima fall out of the measurement window. Note that in the three cases the minima are out of the range of wavelength covered by the tunable laser. Numerical results give transmission minima of $-11 \mathrm{~dB}$ at $1215 \mathrm{~nm}$ in case (a), $-11 \mathrm{~dB}$ at $1155 \mathrm{~nm}$ in case (b) and $-7.5 \mathrm{~dB}$ at $1270 \mathrm{~nm}$ in case (c). In all the cases, the large (>10 dB) contrast are caused by the strong interaction between of the embedded nanoantenna and the waveguide field, which is not attainable in nanoparticle-on-top configurations, in which the nanostructure weakly interacts with the evanescent region of the waveguide mode.

With everything seen in this chapter it has been demonstrated that, through the proposed approach, the efficient excitation of subwavelength metal nanoparticles acting as nanoantennas is achieved. The next step, which will be studied in the next chapter, is the coherent 
control of these nanoantennas with a view to applications that could lead to massive multiplexed coherent all-optical signal processing. 


\section{Chapter 4 Coherent control of plasmonic nanoantennas}

Scattering, absorption and polarization properties exhibited by resonant plasmonic nanoantennas $[17,110,111]$ mainly depend on their physical constitution, which severely limits their use in dynamical applications. Illuminating the nanoantenna by a set of coherent light beams should tremendously modify such properties, thus enabling all-optical dynamic manipulation. However, diffraction inherently makes coherent control of isolated subwavelength-sized nanoantennas highly challenging when illuminated from free-space. In the section 4.2 of this chapter, this limitation is overcome by embedding the nanoantenna into silicon waveguides [112] that provide monolithically-defined paths for multibeam coherent illumination. Inspired by coherent perfect absorption (CPA) concepts [113,114,115], experimentally scattering and absorption modulation is shown, reaching more than one order of magnitude by controlling the phase between two counter-directional coherent guided beams. Moreover, in the section 4.3 of this chapter it is shown that by illuminating a nanoantenna by orthogonal waveguides the polarization of the emitted radiation can be coherently synthesized at will. This should pave the way towards ultrafast manipulation of nanoantennas and all-optical processing without nonlinearities [116117] in an integrated platform.

\subsection{Introduction}

When a monochromatic light beam illuminates a nanoantenna with subwavelength dimensions, part of the light gets either scattered or absorbed, resulting in a certain extinction of the propagating beam (see Figure 4.1(a)). Both scattering - including its polarization state - and absorption will mainly depend on the shape, size and material composition of the nanoantenna. For instance, the response of smallsize (much smaller than the free space wavelength) metallic nanoantennas to single-beam illumination will be essentially that of an electric dipole: light will be scattered forming the well-known "donutshaped" far field pattern with will be polarized along the dipole direction, whilst part of the radiation will be absorbed due to the ohmic losses of the metal [17]. However, the situation may become 
completely different when the nanoantenna is illuminated by a set of coherent beams. Simultaneous illumination of the nanoantenna by means of two counter-directional coherent light beams can result in either suppressed (Figure 4.1(b)) or enhanced (Figure 4.1(c)) absorption and scattering depending on the phase difference between the beams because of the interplay between absorption and interference $[113,114,115,116,117]$. Indeed, when the object is an absorbing film, the regime known as CPA [114] - for which no light exists the system - becomes attainable. Indeed, CPA can even work for subwavelengthlength structures as long as they provide the adequate amount of absorption. For instance, CPA can be achieved when using a subwavelength material film showing 50\% of absorption for a single beam [115,116]. In this case, coherent perfect transmission - the process opposite to CPA - is achievable for a zero-thickness layer.

A key aspect resulting from CPA is that a light beam is affected by another light beam without the presence of nonlinear materials [116].

Therefore, it can be used to perform all-optical modulation of the intensity of a light beam, which can bring different advanced functionalities for coherent light processing [117]. Remarkably, since there are not constraints on the process speed, ultrafast processing beyond $\mathrm{THz}$ rates becomes attainable [118]. But multipath coherent illumination would also enable to modify the response of the nanoantenna in other ways. As shown in Figure 4.1(d), illuminating a nanoantenna by two orthogonal paths would allow to modify the polarization of the scattered radiation. If it is assumed that the nanoantenna response is dominated by its in-plane electric dipolar resonance - which is the case of subwavelength thin metallic disks [94]- then both paths will excite orthogonal transversal dipoles. Then, under a $\pi$ phase difference, circular polarization in the direction of the disk axis becomes attainable, even though the nanoantenna is not chiral. For other symmetries, the polarization response of the nanoantenna in every spatial direction could also be tailored at will by properly choosing the amplitude and phase of each pathway. Whilst many previous works have measured the scattering and absorption of individual nanostructures by strongly focusing free-space beams $[12,119]$, the realization of experiments using multiple beams imposes several challenges. For instance, accurate control of the illumination angles whilst keeping the focusing of all the beams in a certain position is required. Of course, the whole system becomes more complicated as long as the addition of more feeding paths and could be unrealizable to 
control simultaneously a number of nanoantennas, at it may happen in many practical applications.

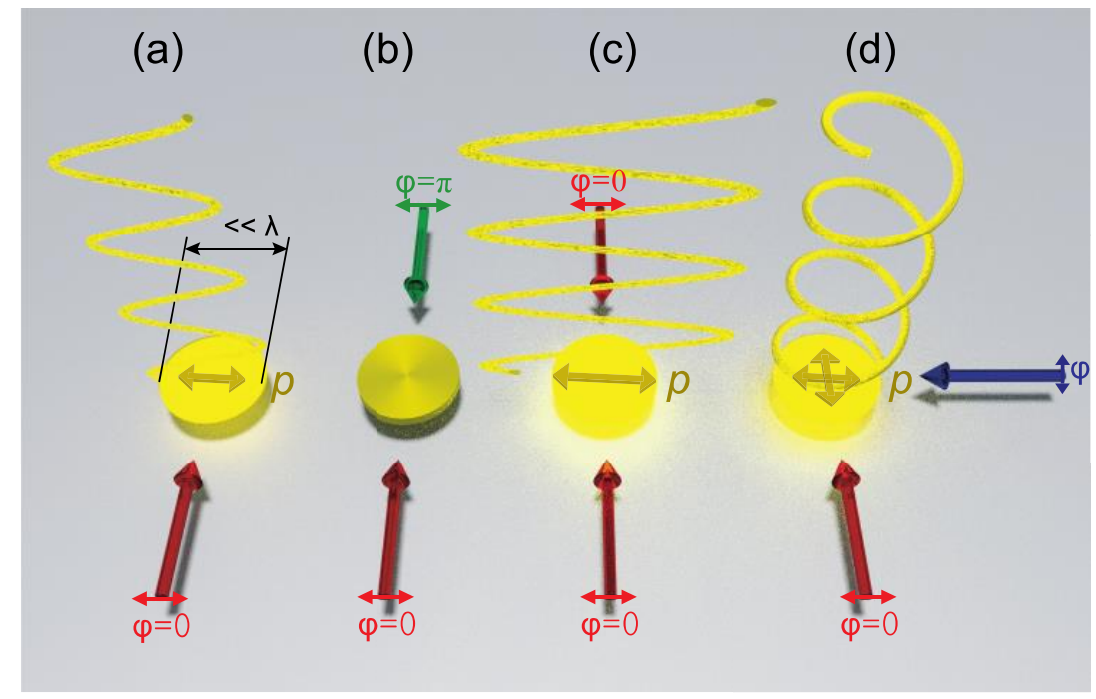

Figure 4.1 Coherent manipulation of the absorption, scattering and polarization of a single nanoantenna. (a) Under single-beam illumination, light is partly scattered and absorbed by the nanoantenna depending on the polarization of the input light and the multipolar response of the nanoantenna. When two coherent, equally polarized and counter-directional beams illuminate the antenna, scattering and absorption are either suppressed (b) or enhanced (c) depending on the phase shift between pathways, whilst polarization of the scattered light is maintained. When the beams form a $90^{\circ}$ angle, linear superposition enables to modify the polarization of the radiated signal.

To overcome this limitation, as in the previous chapter, the idea is to embed the nanoantenna in an integrated circuit and use optical waveguides (with modal sizes of the order of $\lambda / 2 n$, being $\lambda$ the vacuum wavelength and $n$ the refractive index of the waveguide) to illuminate it [112]. If the nanoantenna is well aligned with the waveguide axis and the distance between the waveguide facet and the nanoantenna is deeply subwavelength, there will be a strong overlap between the guided mode and the nanoantenna resonance, which will result in highly efficient excitation of the nanoantenna. In addition, the direction of the illumination signals can be accurately defined by lithography whilst their amplitude and phase can be controlled either by using on-chip modulators or external mechanisms. Remarkably, the use of optical 
waveguides to connect nanoantennas would enable dual wireless/wired optical networks, just mimicking what is widely done in the RF and microwave domains. Moreover, this hybrid plasmonic-photonic approach [120] would enable the simultaneous coherent driving of multiple nanoantennas integrated on a same chip.

\subsection{Coherent control of absorption, scattering and transmission}

\subsubsection{Description of the concept}

The transfer matrix formalism is used to model the response of the 2port system for coherent control of absorption and scattering. In this section, the 1D system schematically depicted in Figure 4.2(a) is considered, although it is a simplified version of the full 3D nanophotonic structure (see Figure 4.3), it will allow to describe qualitatively the main features of the system.

(a)

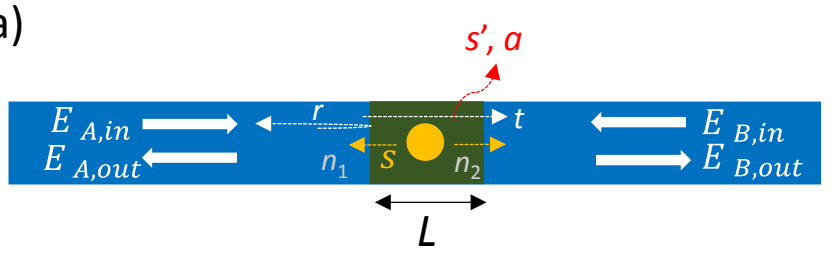

(b)

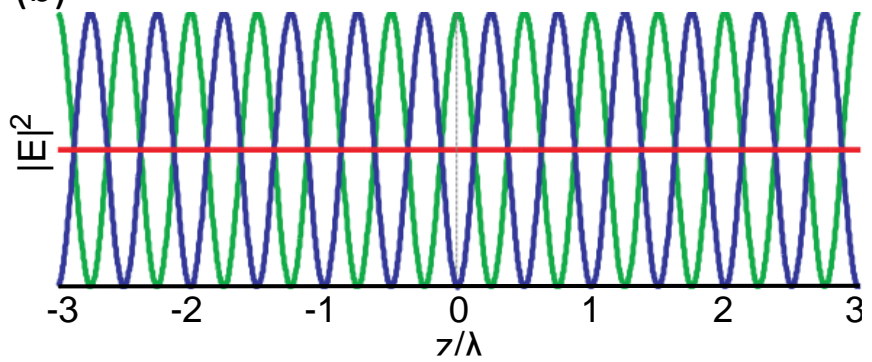

Figure 4.2 (a) Schematic 1D description of the system under consideration. The waveguides are depicted in blue, the gap region in green and the nanoantenna in orange. The different parameters considered in the analytical model are shown. (b) Optical intensity $\left(|E|^{2}\right)$ along the waveguide - without the nanoantenna and for $\mathrm{L}=0$ - for three cases: dual-beam illumination with $\varphi=0$ (green curve) and $\varphi=\pi$ (blue curve) and single beam illumination (red curve). 
The assumption is that the nanoantenna (orange circle) is embedded within a dielectric medium of length $g$ much smaller than the wavelength so that retardation effects are neglected. When illuminated, the nanoantenna scatters part of the radiation into guided modes towards each output port $(s)$. The loss of the system can either be absorption in the plasmonic nanoantenna $(a)$ or out-of-axis scattering $\left(\mathrm{s}^{\prime}\right)$. Notice that $s, s^{\prime}$ and $a$ will be strongly related since they come from the excitation of the nanoantenna from an external electric field. Therefore, it can be written:

$$
\left[\begin{array}{l}
E_{A, \text { out }} \\
E_{B, \text { out }}
\end{array}\right]=\left[\begin{array}{ll}
S_{11} & S_{12} \\
S_{21} & S_{22}
\end{array}\right]\left[\begin{array}{l}
E_{A, \text { in }} \\
E_{B, \text { in }}
\end{array}\right]=\left[\begin{array}{ll}
s+r & s+t \\
s+t & s+r
\end{array}\right]\left[\begin{array}{l}
E_{A, \text { in }} \\
E_{B, \text { in }}
\end{array}\right]
$$

Where $s, r$ and $t$ are wavelength-dependent, complex parameters. In absence of the nanoantenna, the system behaves so that part of the incoming waves is either transmitted $(t)$ or reflected $(r)$ because of the discontinuity between media, which will also cause out-of-axis scattering ( $\mathrm{s}^{\prime}$ ). However, in this case $s=0$ (also $a=0$ ) so Equation (4.1) reduces to:

$$
\left[\begin{array}{l}
E_{A, \text { out }} \\
E_{B, \text { out }}
\end{array}\right]=\left[\begin{array}{ll}
r & t \\
t & r
\end{array}\right]\left[\begin{array}{l}
E_{A, \text { in }} \\
E_{B, \text { in }}
\end{array}\right]
$$

Here, $r$ and $t$ could be obtained analytically by assuming that the gap is a lossy subwavelength-size cavity (the losses comes from the scattering at the waveguide boundaries, which can be high because of the strong confinement in the waveguides). Such values can also be retrieved via full wave numerical simulations, as in Ref. [112], where it was shown that for large $L / \lambda$ ratios (approaching $1 / 2),|t|$ is small $(<0.5)$ and $|r| \approx|t|$. However, as the gap length is reduced or the wavelength is increased, $|t|$ increases and $|r|$ decreases, tending to the expected values $(t=1, r=0)$ when the gap is closed. In this case $(L=0)$, the nanoantenna is completely embedded into a waveguide [62] and Equation (4.1) reduces to a simplest form:

$$
\left[\begin{array}{c}
E_{A, \text { out }} \\
E_{B, \text { out }}
\end{array}\right]=\left[\begin{array}{cc}
s & s+1 \\
s+1 & s
\end{array}\right]\left[\begin{array}{c}
E_{A, \text { in }} \\
E_{B, \text { in }}
\end{array}\right]
$$

Remarkably, Equation (4.3) is identical to Equation (4) in Ref. [121] for the case of ultrathin metasurfaces, which can be considered as composed by an array of nanoantennas all of them simultaneously 
illuminated. Essentially, the described system enables to control such nanoantennas in an independent way.

Now is going to be considered the case in which both paths have the same amplitude but there can be a phase difference $\varphi$ between them (as in the experimental system described later). Then, the matrix transfer formalism leads to:

$$
\left[\begin{array}{l}
E_{A, \text { out }} \\
E_{B, \text { out }}
\end{array}\right]=\left[\begin{array}{ll}
s+r & s+t \\
s+t & s+r
\end{array}\right]\left[\begin{array}{c}
e^{i \varphi / 2} \\
e^{-i \varphi / 2}
\end{array}\right]
$$

Notice that in this case the intensity across the waveguide will be modified locally, with a set of intensity nodes and anti-nodes (Figure 4.2(b)) which is precisely which provides the possibility to tailor the scattering and the absorption of the nanoantennas integrated in the waveguide.

For the study of the modulation of scattering, absorption and extinction it is considered that the nanoantenna response is dominated by a dipolar electric resonance perpendicular to the waveguide axis. So, it is considered also that the input fields are parallel to the nanoantenna electric dipole moment $\vec{p}=p \hat{x}$ in order to achieve an efficient excitation ( $p$ is proportional to the incoming electric field via the nanoantenna polarizability). This can be easily done by exciting the fundamental TE-like mode of the waveguide [112]. Then it is obtained that in the described scheme - assuming equal amplitude feeding signals with a phase shift $\varphi$ between them $-|p| \propto\left|e^{i \varphi / 2}+e^{-i \varphi / 2}\right|=$ $2|\cos (\varphi / 2)|$, so the dipolar moment gets modulated by the phase difference between interfering signals. Notice that similar considerations could be made if the nanoantenna has a multipolar response, as long as the incoming field has electric or magnetic field components able to excite the multipolar moments of the nanoantenna. The nanoantenna extinction (including both out-of-axis $s$ ' and guided $s$ scattering terms as well as the absorption $a$ ) will be proportional to $p$ so all these terms will also become modulated in proportion to $|\cos (\varphi / 2)|$ (or $I_{\mathrm{s}^{\prime}} \propto|\cos (\varphi / 2)|^{2}$ if optical intensity is considered). Thus, for $\varphi=$ $0(=\pi)$ at $\mathrm{x}=0$ there will be constructive (destructive) interference at the position of the nanoantenna and both the scattering and absorption will be maximized (minimized), as shown later in the numerical results.

But not only the nanoantenna extinction can be controlled, also the waveguide transmission (and reflection) is modulated via coherent 
interference in the nanoantenna. Considering a single output from Equation (4.1) it can be obtained

$$
E_{B, \text { out }}=(s+t) E_{\alpha}+(s+r) E_{\beta}
$$

The output intensity can be calculated as [121]:

$$
\begin{aligned}
I_{B, \text { out }} & =E_{B, \text { out }} E_{B, \text { out }}^{*} \\
& =|s+t|^{2} I_{A, \text { in }}+|s+r|^{2} I_{B, \text { in }}+2 \operatorname{Re}\left\{(s+t)(s+r)^{*} E_{A, \text { in }} E_{B, \text { in }}^{*}\right\}
\end{aligned}
$$

Considering the simplified case used in this section, from Equation (4.4) it is obtained:

$E_{B, \text { out }}=(s+t) e^{\frac{i \varphi}{2}}+(s+r) e^{-\frac{i \varphi}{2}}=2 s \cos (\varphi / 2)+t e^{i \varphi / 2}+r e^{-i \varphi / 2}$

and

$$
I_{B, \text { out }}=|s+t|^{2}+|s+r|^{2}+2 \operatorname{Re}\left\{(s+t)(s+r)^{*} e^{i \varphi}\right\}
$$

Equation (4.8) can be also written as

$$
I_{B, \text { out }}=4|s|^{2} \cos ^{2}\left(\frac{\varphi}{2}\right)+|t|^{2}+|r|^{2}+2 \operatorname{Re}\left\{2 \cos \left(\frac{\varphi}{2}\right) s\left(t^{*} e^{-\frac{i \varphi}{2}}+r^{*} e^{\frac{i \varphi}{2}}\right)+t r^{*} e^{i \varphi}\right\}
$$

In Equation (4.8) and (4.9) it is clearly seen that the phase difference between the counter-propagating feeding signals modulates the output intensity of the system. Therefore, the system would be highly appropriate for ultrafast on-chip coherent data processing. However, the intensity modulation becomes more complicated than in the case of the scattering, since now there is a $\varphi$-dependent term that also modulates the reflection and transmission coefficients of the empty gap. Still, it is being possible to design the system so that the output power gets $I_{\gamma} \propto \cos ^{2}(\varphi / 2)$, as in conventional optical modulators (for instance those based on Mach-Zehnder interferometers) if:

- Case I: $t=r e^{-i \varphi}$ and then $I_{B, o u t}=4|s|^{2} \cos ^{2}\left(\frac{\varphi}{2}\right)$. This corresponds to the situation where the single-beam transmission and reflection without the nanoantenna interference destructively and the output signals are exclusively due to the nanoantenna scattering. 
- Case II: $t=r$ and then $I_{B, o u t}=4|s+t|^{2} \cos ^{2}\left(\frac{\varphi}{2}\right)$. This corresponds to the situation where the outputs in each direction has the same amplitude for single beam illumination, so controlling the phase difference such response can be modulated in a sinusoidal way.

Therefore, the proposed system could be engineered to perform as an optical modulator in both of the previous cases.

When the gap size reduces to zero, it is obtained $t=1, r=0$ (Case III) and the previous expressions reduce to those shown in Ref. [121], so $I_{B, \text { out }}=4|s|^{2} \cos ^{2}\left(\frac{\varphi}{2}\right)+1+4 \cos \left(\frac{\varphi}{2}\right) R e\left\{s e^{-\frac{i \varphi}{2}}\right\}$.

Moreover, alike the joint absorption in thin films where scattering can be negligible [122], in this system joint extinction $E$ (out-of-axis scattering plus absorption) can be defined as:

$$
E=1-\frac{I_{A, \text { out }}+I_{B, \text { out }}}{I_{A, \text { in }}+I_{B, \text { in }}}
$$

The regime of coherent perfect extinction (CPE) is attained when $E=$ 1 , which means that both output waves are zero. In general, always there is a way to design the system so that for a certain set of parameters the output intensity falls to 0 . In cases I and II CPE is achieved when $\varphi=\pi$. In Case III, CPE will not only depend on the phase difference $\varphi$ but also on the value of $s$. Remarkably, when $\varphi=0$, which means that there is an antinode at the nanoantenna position, it is obtained that $s=-1 / 2$, which corresponds to the case of an infinitely thin medium with $50 \%$ absorption.

It is also worth analyzing if the system enables coherent perfect transmission (CPT), at least in one transmission path. It is possible to study if the system allows $E_{B, o u t}=E_{A, \text { in }}$ under any condition. From Equation (4.5) it can be obtained that this condition is fulfilled if:

$$
E_{B, i n}=\frac{1-s-t}{s+r}
$$

In this picture, $E_{B, \text { in }}$ can be considered as a bias of the system enabling $\mathrm{CPT}$ in the $\mathrm{A} \rightarrow \mathrm{B}$ direction. Moreover, $E_{B, \text { out }}=0$ if:

$$
E_{B, i n}=\frac{-s-t}{s+r} E_{A, i n}
$$

Which means that the system may potentially be switched without losses, giving rise to an ideally infinite transmission modulation 
contrast $M_{t}$ for guided light, although only along the $\mathrm{A} \rightarrow \mathrm{B}$ path. Getting the same performance along the $\mathrm{B} \rightarrow \mathrm{A}$ path also requires $r=t$, which is one of the possible conditions for CPE.

\subsubsection{Numerical results}

In a real system, the different parameters involved in the 1D scatteringmatrix model discussed above (namely $t, r$ and $s$ ) will be strongly dependent on the chosen materials, the geometry and size or the structures and the wavelength. Owing to this complexity, full 3D numerical simulations have been used to better understand the main features of this system. The starting point consists of the design seen in the previous chapter, considering the coherent control of the scattering and absorption of an individual nanoantenna placed in a subwavelength gap separating to silicon waveguide (see Figure 4.3).

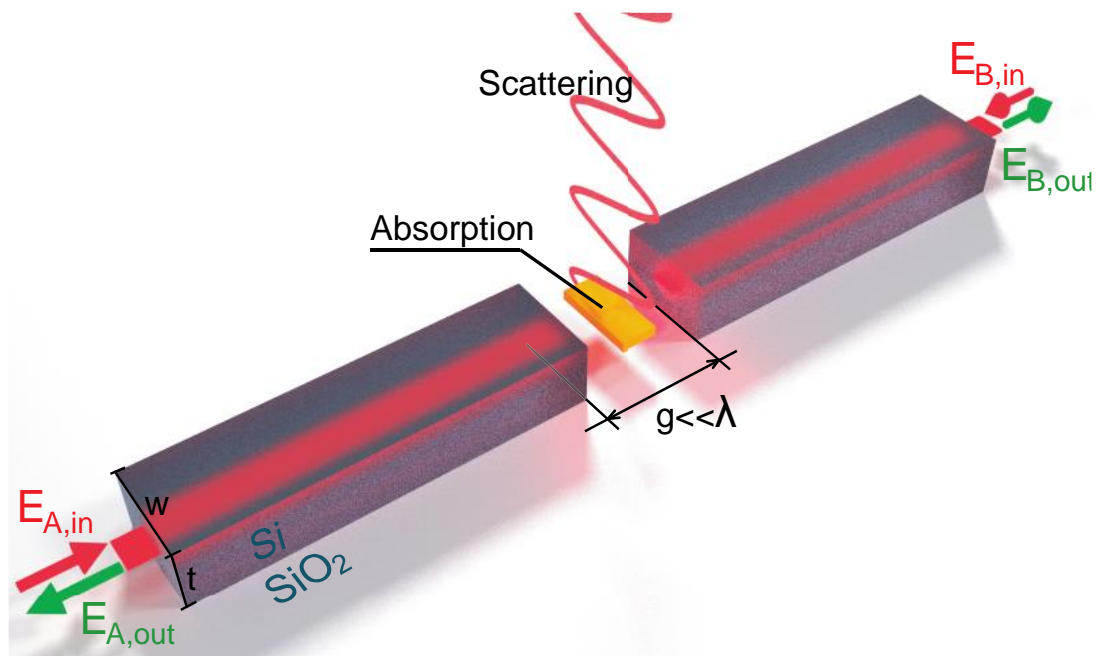

Figure 4.3 On-chip coherent control of absorption and scattering of a single nanoantenna. Sketch of the proposed structure.

Two monochromatic counter-propagating guided waves are injected through ports A and B so that they can simultaneously illuminate the nanoantenna. As previously done, a metallic nanostrip supporting an electric dipole plasmonic resonance along the $x$ axis is considered, which can be efficiently excited when the waveguides are fed by the fundamental even or TE-like mode of the waveguide. For the numerical 
calculations, a gold nanostrip is considered as the isolated nanoantenna. The nanostrip dimensions $(320 \times 125 \times 40 \mathrm{~nm})$ were selected so that is supports a bright dipolar resonance in the telecom regime when excited with an electric field aligned with its major axis (see scattering and absorption cross sections in Figure 4.4).

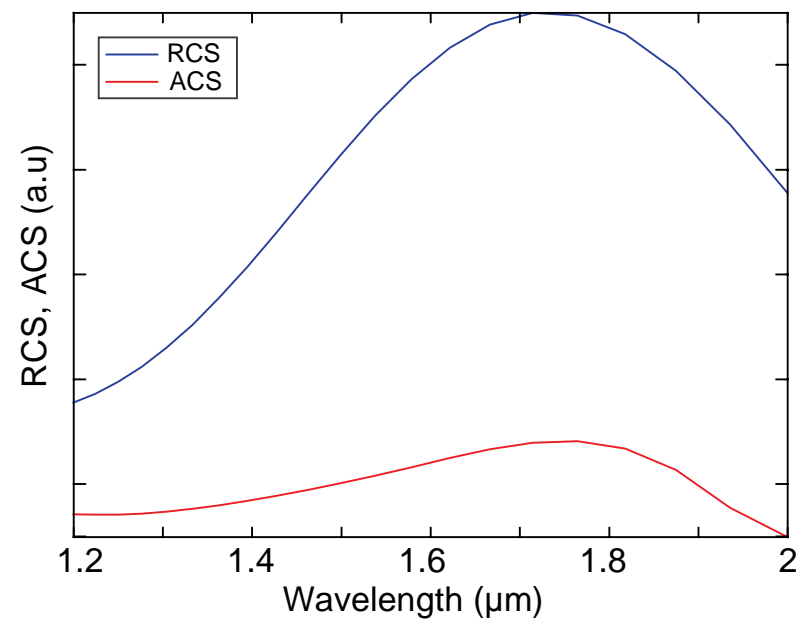

Figure 4.4 Scattering and absorption cross section of the plasmonic nanoantenna under study, consisting of a gold nanostrip with dimensions $320 \times 125 \times 40 \mathrm{~nm}$.

Two silicon waveguides with rectangular cross-section $\left(250 \times 400 \mathrm{~nm}^{2}\right)$ are considered, separated by a gap $(g=300 \mathrm{~nm})$ where the nanoantenna is to be inserted. The whole system (waveguides and nanoantenna) was surrounded by silica. The waveguides are excited to propagate only the fundamental TE-like mode, having a main field component parallel to the nanoantenna axis. Therefore, the electric field in the gap overlaps with the nanoantenna dipolar moment (along $x$ ) and the nanoantenna is efficiently excited as seen in Chapter 3.

In the experimental system, both inputs have the same amplitude $\left(E_{i n}=1\right)$ but there will be a wavelength-dependent phase shift $\varphi$ between them because of the different path lengths they have travelled along the waveguides. If the path length difference is $\Delta L$, the effective index of the waveguide mode is $n$ and the wavelength is $\lambda$, the phase shift between both input ports can be simply obtained as $\varphi=2 \pi n \Delta L / \lambda$. Figure 4.5 shows the effective index of the two fundamental modes of the employed waveguides calculated using full wave simulations. 


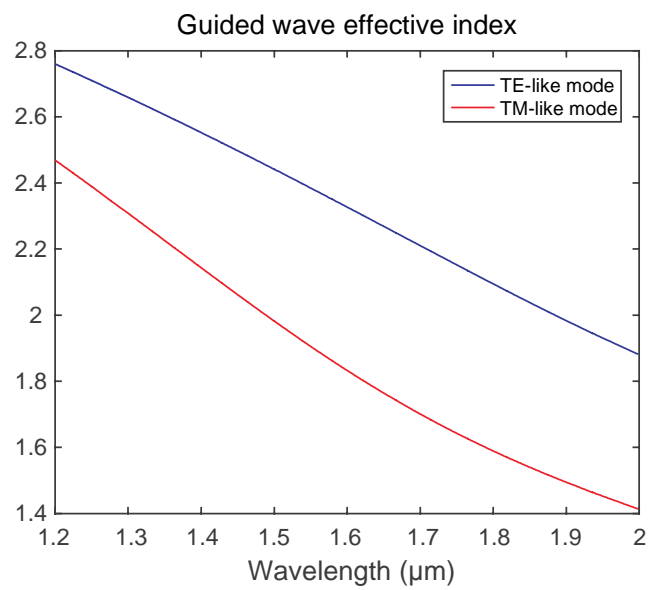

Figure 4.5 Dispersion relation of the fundamental TE-like and TMlike modes of a silicon waveguide with cross-section of $400 \times 250$ $\mathrm{nm}$ and surrounded by silica.

Thus, for the numerical calculations of the modulation, it has been considered the path length difference of the sample, $\Delta L=21 \mu \mathrm{m}$. Figure 4.6(a) depicts the numerically calculated spectra of the recorded normal scattering along the $+y$ axis when the nanoantenna is inserted under four different conditions: single-beam illumination ${ }^{2}$ and dual-beam illumination with same amplitude but $\varphi=0, \varphi=\pi$ and wavelengthdependent phase shift $\varphi$. If it is considered that the scattering of the nanostrip displays a donut-like shape (somewhat deformed due to the anisotropy of the whole system) with is axis along the nanostrip axis, such spectrum can be considered as a good approximation to out-ofaxis scattering s'. As expected from Figure 4.1(a-c) as well as from the previous considerations on the modulation of the scattering, it is observed that the coherent scattering is either enhanced or suppressed in comparison with the single-beam situation depending on the phase difference between signals. Indeed, an almost-complete suppression of scattering can be observed in comparison to the single beam case at the exact frequency for which $\varphi=\pi$.

This control on the scattering will also be well appreciated in Figure 4.6(c-d), which show respectively numerical simulations showing the scattered field under the situations of constructive (c) or destructive (d)

\footnotetext{
${ }^{2}$ Single-beam illumination considered in this chapter corresponds to the case seen in the previous chapter and will be used as a control for the study of the coherent dual-beam feeding.
} 
interference, respectively. It is important to highlight that in both situations the optical amplitude reaching the nanoantenna from each side is the same, but the phase difference results in constructive or destructive interference that ultimately switches on and off the dipolar resonance of the nanoantenna. Note that in this ideal picture with perfect symmetry with respect to the plane $x=0$, the scattering modulation depth $M_{s}=\max \left(I_{\mathrm{s}^{\prime}}\right) / \min \left(I_{\mathrm{s}^{\prime}}\right)$ will be infinite since when the phase difference is $\varphi= \pm \pi$ the electric field along the nanoantenna and all the related processes such as scattering (Figure 4.6(a)) or absorption (Figure 4.6(b)) are cancelled ${ }^{3}$.
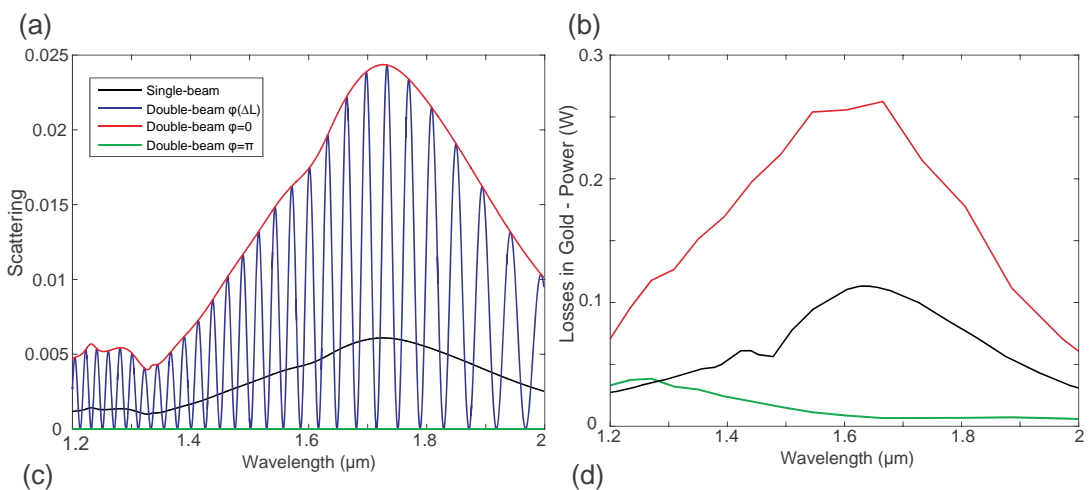

(C)

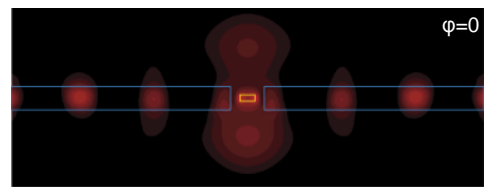

(d)

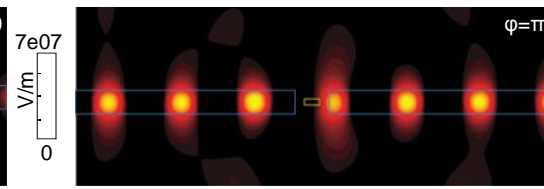

Figure 4.6 (a) Spectral modulation of scattering. Calculated intensity modulation (in blue) of the scattering under illumination from two waveguides with a length difference $\Delta \mathrm{L}=21 \mu \mathrm{m}$, which is the case of the fabricated and measured sample. (b) Numerically calculated nanoantenna absorption under dual-beam (for both $\varphi=0$ and $\varphi=\pi$ ) and single beam illumination conditions. (c) Numerical simulation shown the cross-section of the fields for enhanced scattering. (d) Numerical simulation shown the cross-section of the fields for suppressed scattering.

\footnotetext{
${ }^{3}$ It should be noted that breaking the translational symmetry of the waveguides because of the gap will also cause out-of-axis scattering even when the nanoantenna is not present.
} 
Numerical simulations of the transmission response were also performed. The values of $r$ and $t$ can be easily obtained by simulating the system without the nanoantenna. After that, $s$ can be obtained by simulating the structure with nanoantenna. Notice that the conditions required for Cases I or II are not met. Therefore, the dependence of the transmission response on the phase difference will be not straightforward. This is seen on Figure 4.7, which depicts the transmission spectrum under the conditions previously considered when representing Figure 4.6.

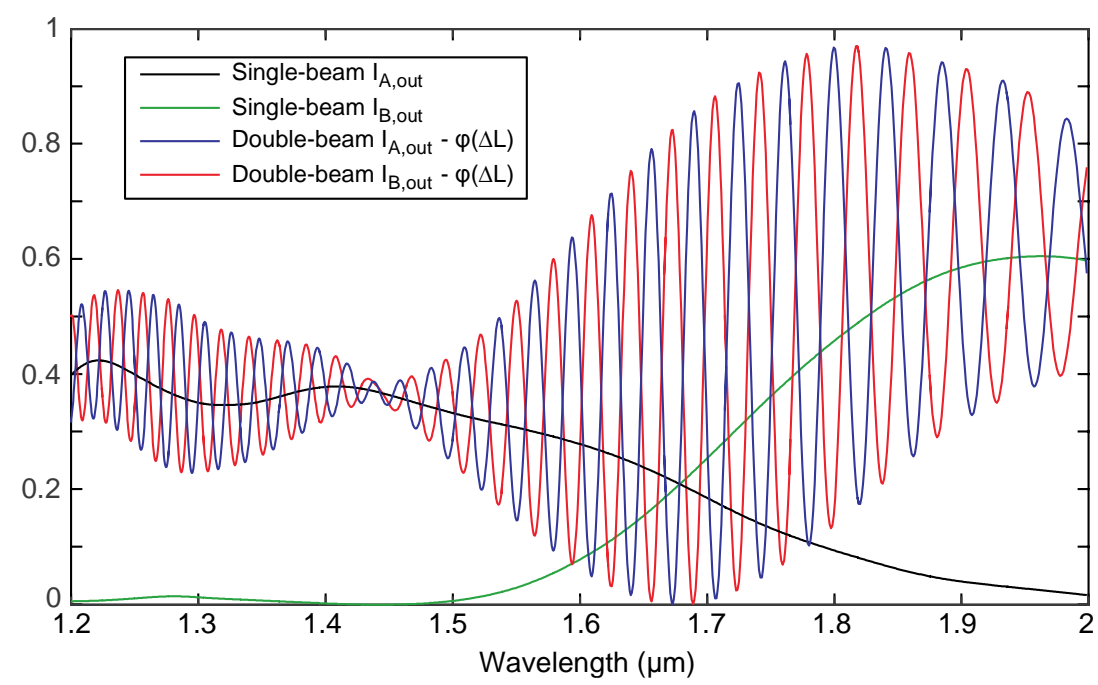

Figure 4.7 Spectral modulation of transmission. Calculated intensity modulation (in red-blue) transmission under illumination from two waveguides with the path length difference of the fabricated sample $\Delta \mathrm{L}=21 \mu \mathrm{m}$. The spectra have been obtained by using the values $r, s$ and $t$ obtained from the simulations and then applying the analytical described above. It can be seen that the dispersion-induced phase shift produces a set of maxima and minima in the scattering spectrum, being the average curve the scattering of the nanoantenna under single-beam illumination.

So, the interference at the nanoantenna position also enables modulation of the on-chip transmission and reflection paths. In this case, the dependence of the output intensity $\left(I_{\gamma}\right)$ with respect to the phase shows a more intricate behavior than in the case of the scattering. Still, the system could be tailored so that $I_{\gamma} \propto \cos ^{2}(\varphi / 2)$, which 
mainly requires $|r|=|t|$. Noticeably, the regime of coherent perfect extinction (CPE), the equivalent to $\mathrm{CPA}$ in a system with strong scattering as the one under consideration, becomes attainable under the certain circumstances described before. Figure 4.8 shows the calculated extinction spectra as a function of the wavelength for the experimental system. It can be observed that there are regions $(\lambda=1.33 \mu \mathrm{m}$ and $\varphi=$ $180^{\circ}$ ) where the joint extinction $E=0.69$, meaning that the power exiting the system is only $31 \%$.

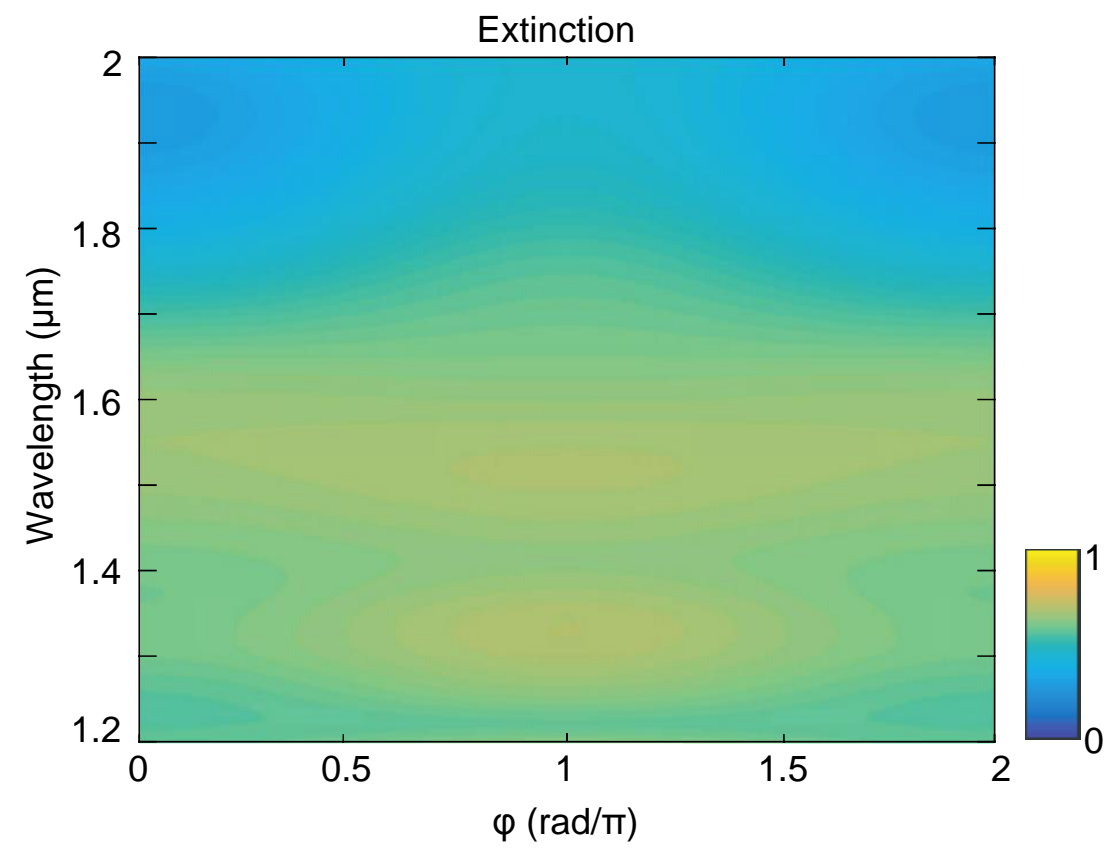

Figure 4.8 Simulated joint extinction $\mathrm{E}$ as a function of both the wavelength $\lambda$ and the phase different between counter-propagating signals.

The two output ports intensity variation, as a function of wavelength and phase difference between counter-propagating signals are shown in Figure 4.9. Maximum and minimum transmission can be observed due to the modulation produced by the phase difference.

Therefore, by changing the phase shift at that wavelength, modulation depths for on-chip transmission $\left(M_{\mathrm{t}}\right)$ can be very large (easily exceeding one order of magnitude), as shown in Figure 4.9. Note that although the outputs in each direction are out of phase (and therefore the maximum 
and minimum do not coincide), the ratio between maximum and minimum for each wavelength coincides, which means that the modulation depth for on-chip transmission $\left(M_{\mathrm{t}}\right)$ is the same for both output ports.
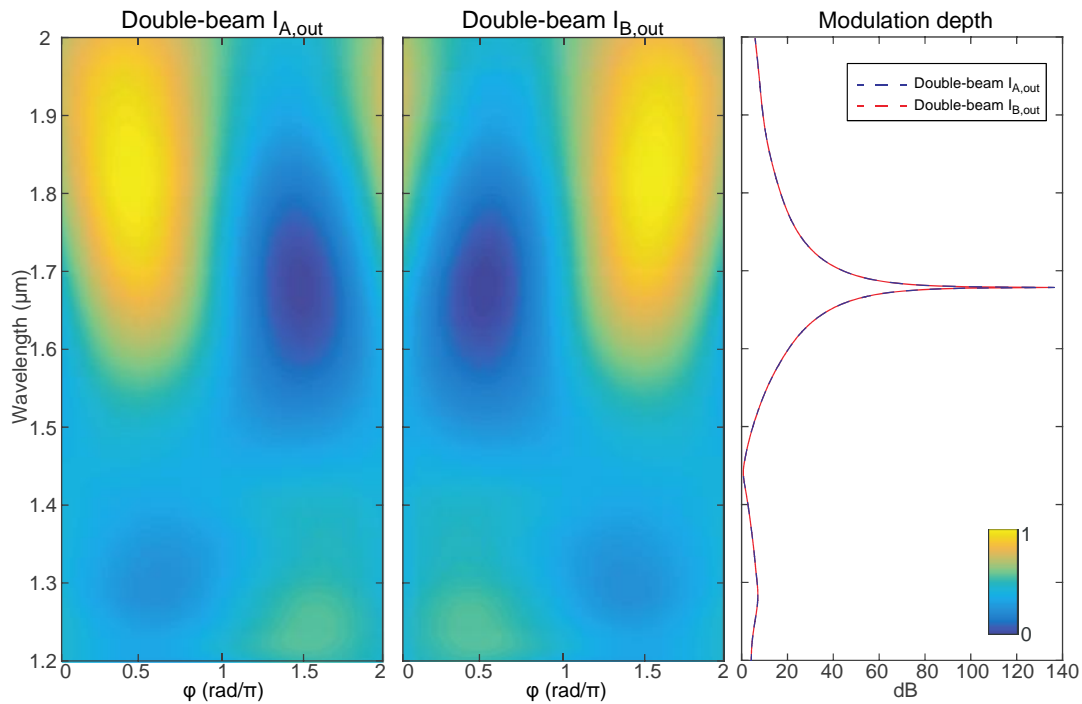

Figure 4.9 Output intensity of the $\mathrm{A} \rightarrow \mathrm{B}$ path $\left(\mathrm{I}_{\mathrm{B}, \text { out }}\right)$ and $\mathrm{B} \rightarrow \mathrm{A}$ path $\left(\mathrm{I}_{\mathrm{A}, \text { out }}\right)$ as a function of both the wavelength $\lambda$ and the phase difference between counter-propagating signals. Maximum transmission modulation depth $\mathrm{M}_{\mathrm{t}}$ as a function of the wavelength.

\subsubsection{Experimental results}

In Figure 4.10 it is depicted the experimental setup. Coherent light was generated in a tunable laser (SANTEC TSL-210F), covering a wavelength range of $1350 \mathrm{~nm}$ to $1600 \mathrm{~nm}$. After passing through a polarization controller that allowed for configure the TE-like mode, light was coupled to the sample through a lensed fiber. Still, the system allows for performing measurements of the normal scattering as well as the transmitted on-chip intensity. 


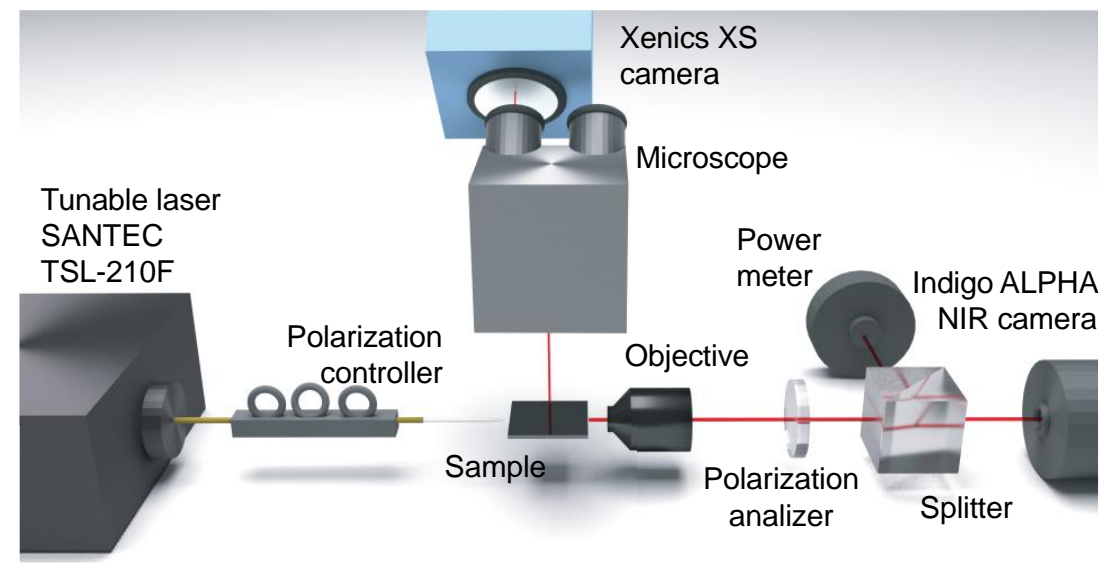

Figure 4.10 Experimental setup. A tunable laser and polarization controller are used to configure the input light, which is coupled to the sample trough a lensed fiber. Using this setup, both transmission and scattering of the nanoparticle is captured. In the vertical plane, a microscope-IR camera combination is used for capturing the scattered light. At the same time, in the horizontal plane transmitted light is collected by an objective, to be divided in two orthogonal paths thanks to which the light spot is observed with an IR camera and its intensity is measured simultaneously with a power meter.

Normal scattering (vertical output light) was measured using an IR camera (Xenics XS) coupled to the eyepiece of a microscope. The transmission (horizontal output light) was split into two beams in order to simultaneously display the light spot and measure the output guided power. In the fabricated sample (see Figure 4.11), the previously described excitation conditions (counter-propagating signals having equal amplitude but phase shifted by $\varphi$ ) are achieved by splitting coherent light propagating through a single waveguide into two different waveguides that are then bent to finally illuminate the nanoantenna in opposite directions from ports $\mathrm{A}$ and $\mathrm{B}$ (blue path in Figure 4.11). Notice that the photonic circuits also enable to perform measurements under single-beam illumination conditions (red path in Figure 4.11). The nanoantenna is perfectly embedded in the gap, though there is a small misalignment $(\sim 20 \mathrm{~nm})$ with respect to the waveguide axis, and its dimensions $(346 \times 40 \times 135 \mathrm{~nm})$ are different from simulation case as a result of inaccuracies in the second lithography step (fabrication details are described in Methods). 


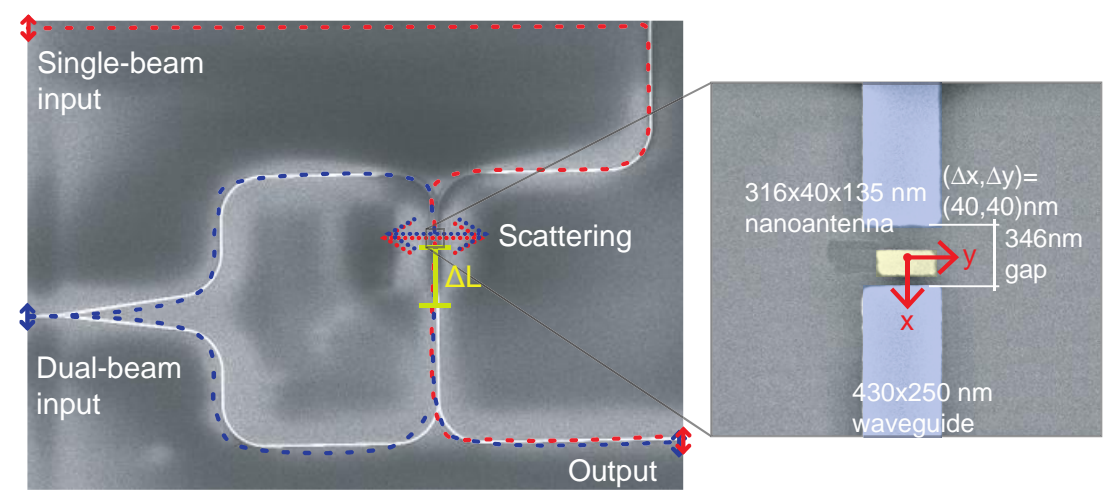

Figure 4.11 SEM images of the sample configuration. The image on the left shows the configuration of the sample, where both single-beam (red path) and dual-beam (blue path) excitation can be achieved for the same nanoantenna. The image on the right shows the dimensions of the fabricated nanoantenna, which are $346 \times 40 \times 135 \mathrm{~nm}$, with a misalignment from the gap centre of $(\Delta \mathrm{x}, \Delta \mathrm{y})=(40,40) \mathrm{nm}$.
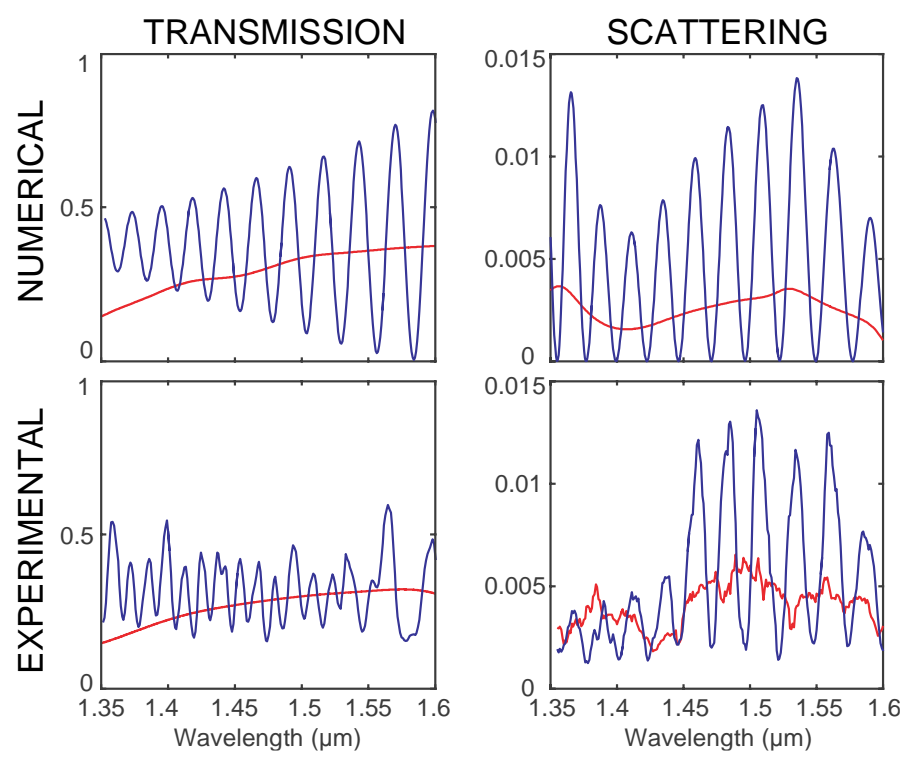

Figure 4.12 Experimental (bottom row) and numerical (top row) results of the fabricated sample. The response to the single-beam excitation is depicted in red, while the dual-beam excitation response is depicted in blue. Both scattering and transmission response have been considered. 
By feeding the chip via the single-beam (dual-beam) input, both the scattering and transmission of the nanoantenna for the single-beam (dual-beam) illumination case are measured. Results of this measurements in comparison with numerical results are shown in Figure 4.12. Numerical results have been recalculated for the dimensions obtained in fabricated samples. In Figure 4.12 it is depicted the modulation due to the dual-beam feeding (red), both in transmission and scattering, as well as the response of the nano-antenna compared to the single-beam excitation (blue).

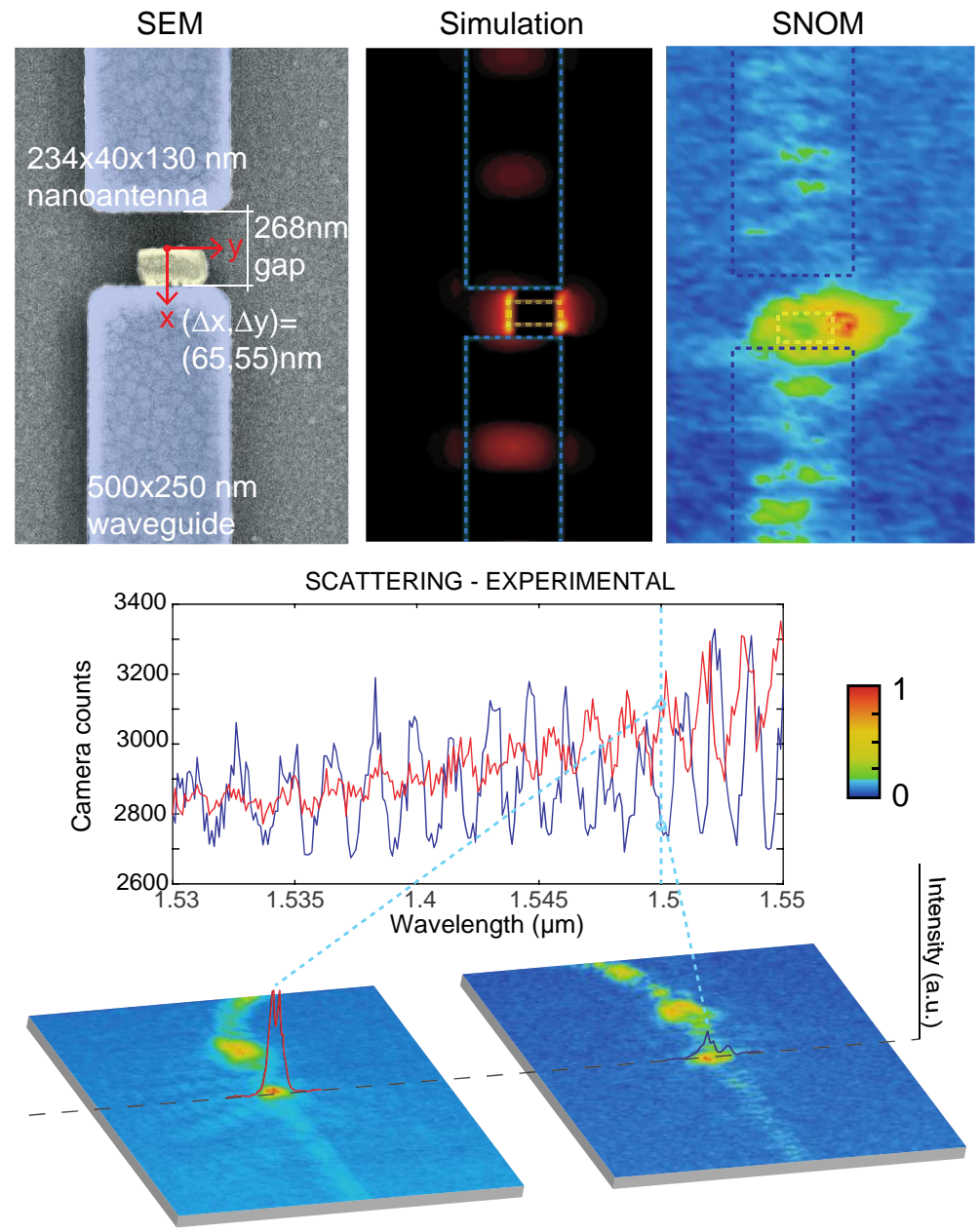

Figure 4.13 Second sample with the same circuits as in Figure 4.11 but with $\Delta \mathrm{L}=300 \mu \mathrm{m}$ and $(\Delta \mathrm{x}, \Delta \mathrm{y})=(65,55) \mathrm{nm}$. Upper panel shows the SEM image and the simulations and near-field measurement at $\lambda=1,550 \mathrm{~nm}$. Mid panel shows the far-field 
normal scattering for both single-beam and dual-beam illuminations. Near field measurements in a $20 \times 20 \mu \mathrm{m}$ region at $\lambda$ $=1,550 \mathrm{~nm}$ for single-beam and dual-beam illumination are detailed in the bottom panel.

Another sample with $\Delta \mathrm{L}=300 \mu \mathrm{m}$ mas fabricated and near-field measurements were performed in addition to the far-field scattering (see Figure 4.13). Suppression of the nanoantenna near-field enhancement for dual-beam illumination at a destructive-interference wavelength is clearly seen. Near-field measurements (see Methods) also confirm the nanoantenna-gap misalignment observed in the SEM images, as well as the different amount of light in each waveguide because of the interference. The good agreement between numerical and experimental results is remarkable.

\subsection{Coherent synthesis of scattering polarization}

In this section it is considered the case of a thin metal disk with revolution symmetry around the z-axis supporting infinite degenerate electric dipolar resonances as a suitable nanoantenna for generating and receiving any polarization state (see the general scheme in Figure 4.1(d)). The same analysis could be performed for nanoantennas with fourfold $\mathrm{C}_{4 \mathrm{v}}$ symmetry (for instance, a nanocross), so that their response is dominated by electrical dipoles with identical strengths but pointing along the $\mathrm{x}$ and $\mathrm{y}$ axis respectively. However, the same principle could be applied to arbitrary shape nanoantennas showing a non-negligible electric dipole along the main axes. The nanoantenna is simultaneously fed by two orthogonal silicon waveguides as shown in Figure 4.14. It is assumed that the nanoantenna is centered with the optical axes of the waveguides cross. The distance between the nanoantenna and each waveguide facet could be engineered to minimize reflection [123].

\subsubsection{Description of the concept}

If the TE-like mode propagates along the feeding waveguides, two electric dipoles will be induced on the nanoantenna: $\vec{p}_{A} \propto$ $\alpha_{d i s k} \beta_{x} E_{A, i n} \hat{x}$ and $\vec{p}_{B} \propto \alpha_{d i s k} \beta_{y} E_{B, i n} \hat{y}$, where $\alpha_{d i s k}$ is the nanoantenna polarizability and $\beta_{x}\left(\beta_{y}\right)$ is the efficiency of excitation of the nanoantenna electric dipole along $x(y)$. Here the effect of the 
longitudinal fields carried by the guided modes is neglected, which is a good assumption provided that the nanoantenna is subwavelength.

For a perfectly symmetric structure, $\beta_{x}=\beta_{y}$ and the nanoantenna radiation in all spatial directions will be therefore a linear superposition of radiation of these two dipoles.

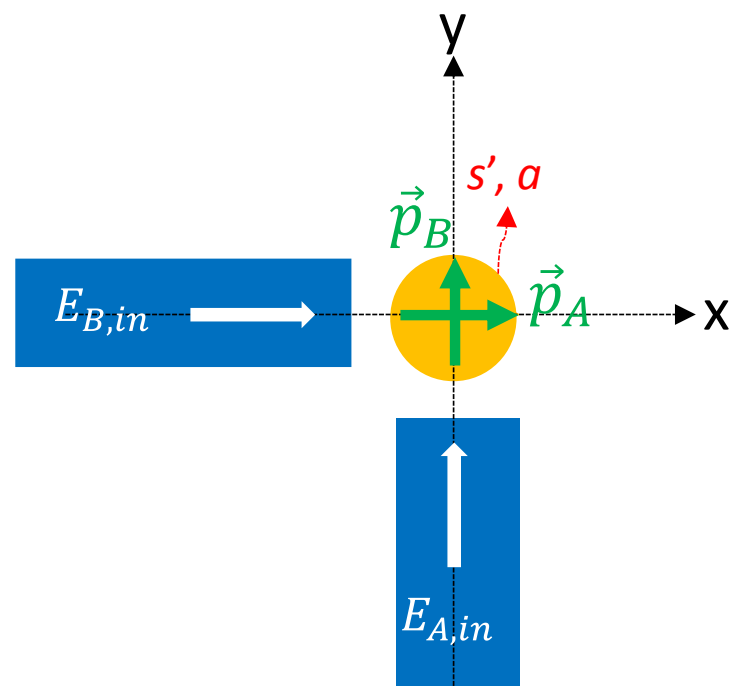

Figure 4.14 Scheme of the coherent approach for synthesis of polarization using an on-chip disk nanoantenna fed by orthogonal waveguides.

In the particular case of the emitted radiation along the z-axis (normal to the chip plane), its electric field can be written as:

$$
\boldsymbol{E}_{\boldsymbol{r}} \propto \alpha_{d i s k} \beta\left(E_{A, i n} \hat{x}+E_{B, i n} \hat{y}\right)=\alpha_{d i s k} \beta\left(\left|E_{A, i n}\right| e^{j \varphi_{A}} \hat{x}+\left|E_{B, i n}\right| e^{j \varphi_{B}} \hat{y}\right)
$$

Where it is assumed the symmetric case $\beta_{x}=\beta_{y}=\beta$ depicted in the figure. Essentially, Equation (4.13) defines a superposition of two orthogonal plane waves whose amplitudes and phases are defined by the amplitudes and phases of the guided signals. Therefore, any polarization state on the Poincare sphere becomes attainable. For instance, linearly polarized modes along $x(y)$ is accomplished when $E_{B, \text { in }}=0\left(E_{A, \text { in }}=0\right)$ or forming a $\pm 45^{\circ}$ with respect to the $x z$ plane when $\left|E_{A, i n}\right|=\left|E_{B, \text { in }}\right|$ and $\varphi_{A, \text { in }}=\varphi_{B, \text { in }} \pm \pi$. Circular polarizations are attained when $\left|E_{A, \text { in }}\right|=\left|E_{B, \text { in }}\right|$ and $\varphi_{A, \text { in }}=\varphi_{B, \text { in }} \pm \pi / 2$. Other 
relations will give the whole set of elliptical polarizations on the Poincaré sphere. In the case of asymmetric excitation, then $\beta_{x} \neq \beta_{y}$. All the polarization states are still attainable but the efficiency will be lower, indeed proportional to the minimum of $\left(\beta_{x}, \beta_{y}\right)$.

\subsubsection{Numerical results}

Equivalent to the previous section, fabricated chips have been designed to have $\left|E_{A, \text { in }}\right|=\left|E_{B, \text { in }}\right|$ and $\varphi_{A, \text { in }}=\varphi_{B, \text { in }} \pm \varphi$, where $\varphi=2 \pi n \Delta L / \lambda$.

Again, the value of $\Delta \mathrm{L}=19 \mu \mathrm{m}$ obtained in the fabricated sample will be considered for the numerical calculations, which will provide with phase variation dependent on the dispersion relation of the waveguide modes (Figure 4.5). By using orthogonal illumination paths (see Figure 4.15), it also becomes feasible to tailor the polarization of the emitted radiation in a coherent way. The nanodisc dimensions (diameter $\mathrm{D}=400 \mathrm{~nm}$ and thickness $\mathrm{t}=40 \mathrm{~nm}$ ) were selected so that it supports a bright dipolar resonance in the telecom regime (see scattering and absorption cross sections in Figure 4.16(a)).

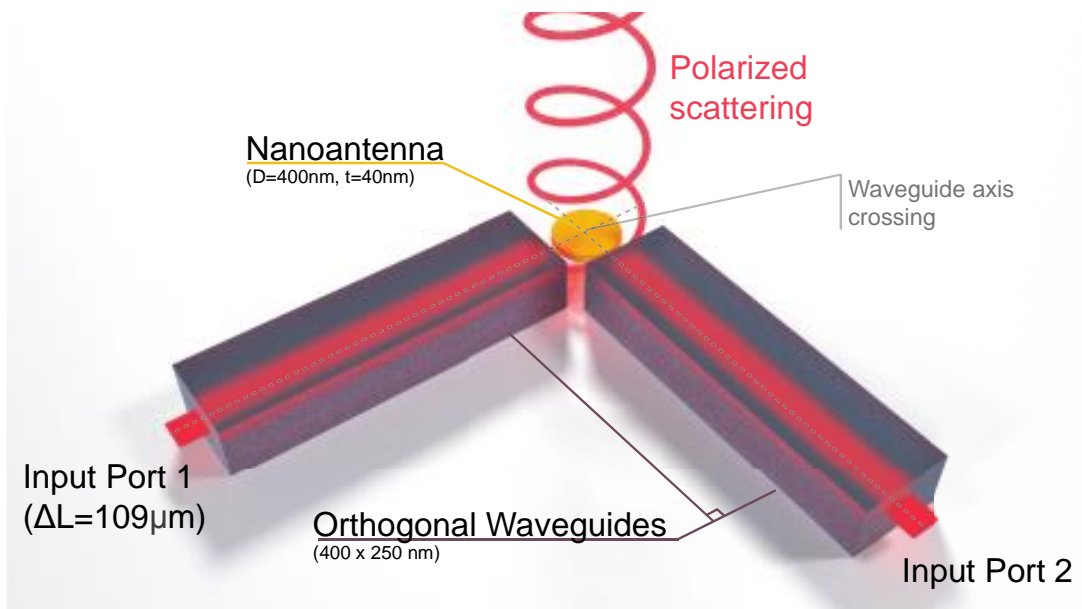

Figure 4.15 Coherent polarization synthesis. Sketch of the structure, in which two orthogonal waveguides illuminate an $\mathrm{Au}$ disk nanoantenna.

Indeed, assuming a nanoantenna with rotational symmetry around the $\mathrm{z}$ axis, by linear superposition the nanoantenna radiation in the normal direction will be proportional to $E_{A, i n} \hat{x}+E_{B, i n} \hat{y}$, so any polarization state can be attained. Numerical results of the polarization synthesis 
considering the scenario described in Equation (4.13) is depicted in Figure 4.16(b). Notice that perfectly circular polarization is achievable.

(a)

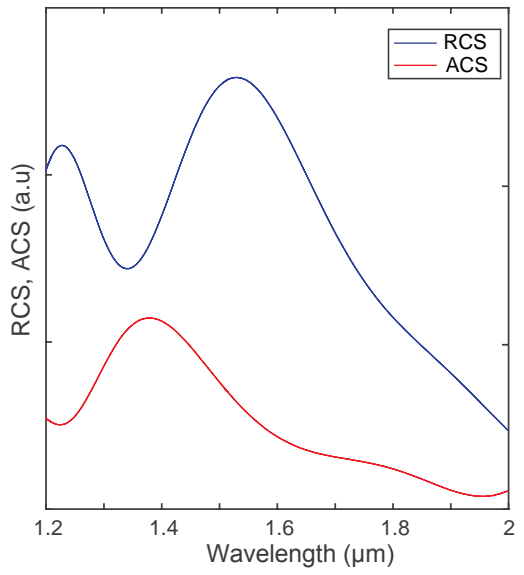

(b)

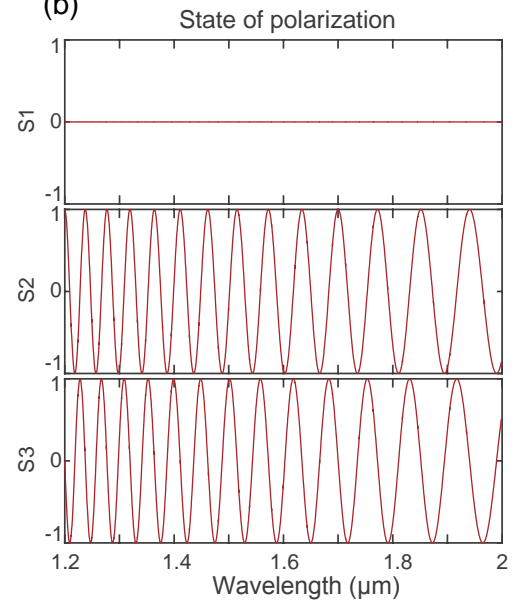

Figure 4.16 (a) Scattering and absorption cross section of the plasmonic nanoantenna under study, consisting of a gold nanodisc with dimensions $\mathrm{D}=400 \mathrm{~nm}$ and $\mathrm{t}=40 \mathrm{~nm}$. (b) Numerically calculated response of the system scattering polarization, in terms of Stokes parameters $S_{1}, S_{2}$ and $S_{3}$.

\subsubsection{Experimental results}

In the fabricated device, an Au disk nanoantenna is illuminated by two waveguides (see SEM image in Figure 4.17) supporting the fundamental TE mode so that they carry the same amplitude but there is a phase difference between them because of the waveguide dispersion (as in the case shown in Figure 4.12, they come from a Yjunction and there is a path length difference $\Delta \mathrm{L}=19 \mu \mathrm{m}$ between them). For experimental measurements, the same setup as in previous section has been used (see Figure 4.10), with the peculiarity that in this case only the scattering light has been collected. In addition, a polarization filter and a quarter-wave plate have been added to the vertical path to characterize the state of polarization. For this, the Stokes parameters have been taken into account, and the scattering output powers have been measured in each of the components that form the basis of Stokes parameters reference system (i.e. linearly polarized polarization at 0 , 45, 90 and 135 degrees and right and left handed circular polarizations). 


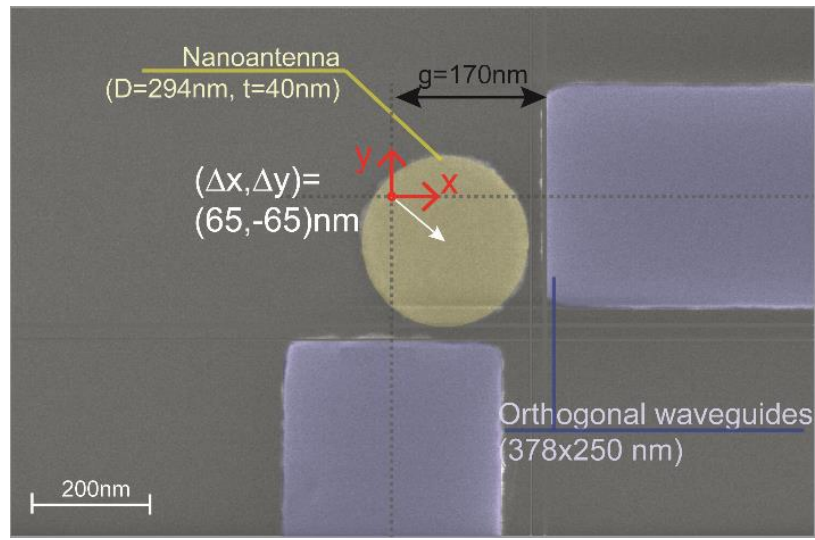

Figure 4.17 SEM image of the fabricated sample. The orthogonal waveguides cross section is $378 \times 250 \mathrm{~nm}$, separated $170 \mathrm{~nm}$ from the crossing of the waveguides axis. The nanodisc diameter is 294 $\mathrm{nm}$ with $40 \mathrm{~nm}$ thickness, and its center is displaced $(\Delta \mathrm{x}, \Delta \mathrm{y})=$ $(65 \mathrm{~nm},-65 \mathrm{~nm})$ from the axis center because of fabrication inaccuracies.

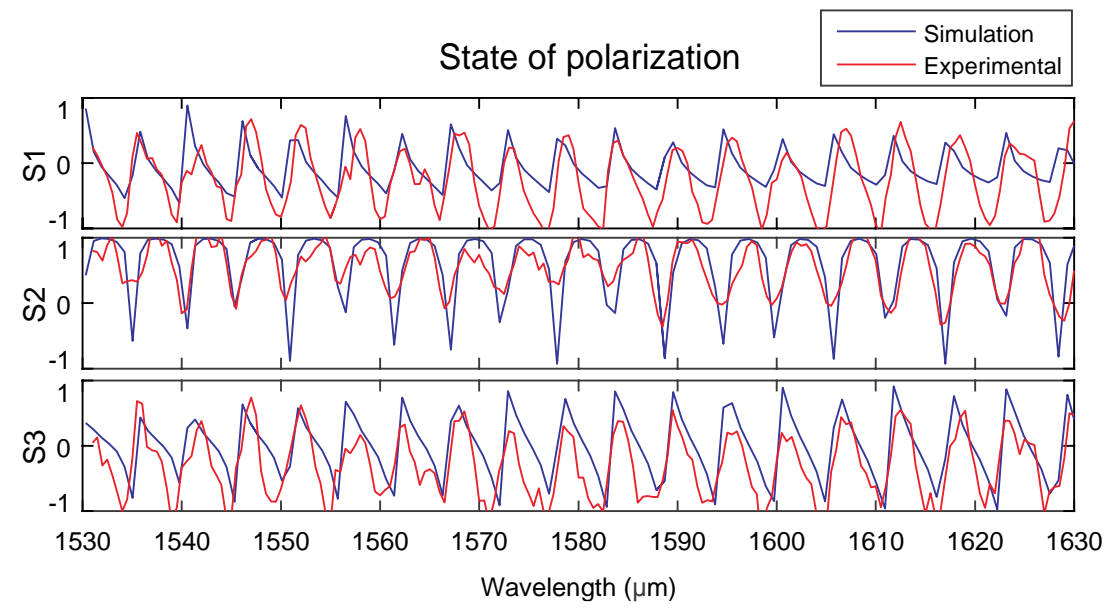

Figure 4.18 Experimental (red) and numerical (blue) results of the state of polarization of the normally-scattered radiation at different wavelengths. The state of polarization is depicted using the Stokes parameters $S_{1}, S_{2}$ and $S_{3}$.

The results of these measurements are depicted in the Figure 4.18, where the experimental results (red) are compared with the numerical results (blue) calculated with the fabricated sample parameters. Note that the inaccuracies obtained in the sample greatly affect the 
synthesized polarization. The reason for this is that the particle has been misaligned slightly from the axis center of the waveguides, and therefore components beyond the electric dipole considered in the ideal case are being excited. For all this, the generated polarization states are limited but still the device is able to radiate different polarizations in good agreement with numerical simulations. Noteworthy, the nanoantenna is able to generate circular polarization even though it is not chiral, which is a clear advantage of the coherent feeding.

In the described samples, the phase different between the illuminating signals was achieved via waveguide dispersion for simplicity. Remarkably, such a phase difference may also be attained in a dynamic way by electro-optical or all-optical modulation of the waveguide modes. That would allow coherent manipulation of either the scattering or the polarization of the nanoantenna at a single wavelength at speeds well above $1 \mathrm{GHz}$. However, tuning the resonant wavelength is not possible in the coherent scheme since it would require to change dynamically the size, shape or permittivity of the nanoantenna (which can be done otherwise using mechanical [124] or electrical means [125]). 


\section{Chapter 5 \\ Optimal Stokes polarimetry implemented on silicon nanoantennas}

Full measurement of the polarization of light at the nanoscale is expected to be crucial in many scientific and technological disciplines. Ideally, such measurements will require miniaturized Stokes polarimeters able to determine polarization non-destructively, locally and in real time. For maximum robustness in measurement, a key aspect when dealing with faint signals, the polarimeters should also operate optimally. Recent approaches making use of plasmonic nanostructures or metasurfaces are not able to fulfil all these requirements simultaneously. In this chapter, it is proposed and demonstrated a method for subwavelength-footprint Stokes nanopolarimetry based on spin-orbit interaction of light. The method, which basically consists on a subwavelength scatterer coupled to a (set of) multimode waveguide(s), can fully determine the state of polarization satisfying all the previous features. Remarkably, the nanopolarimetry technique can be operate optimally over a broad bandwidth. Although here experimentally demonstrated on a silicon chip at telecom wavelengths, SOI-based nanopolarimetry is a universal concept to be applied in any wavelength regime or technological platform.

\subsection{Introduction}

While apparently unrelated, the propagation direction and the polarization of a light beam may be strongly connected via spin-orbit interactions (SOI) of light $[126,67,127,128,129,130,131,132,133,134,135,136]$. Indeed, SOI is ultimately responsible for the unidirectional propagation of guided waves controlled by the spin of an excitation source [66,137], as shown in Chapter 2. Spin-controlled directional guiding of light has been demonstrated in a wide variety of guiding systems, including metal plates [66], optical fibers [69] and integrated waveguides [77,138]. In these systems, SOI takes place when placing a certain emitting (i.e., a quantum dot) or scattering (i.e. a subwavelength scatterer) element that acts as a spin-controlled point-like source in a region where the waveguide mode has a non-negligible local transverse spin [59]. Unlike plane waves (exclusively carrying longitudinal spin), evanescent waves 
present in guided fields display a strong transverse spin that is locked to their momentum, so spin-controlled directional guiding can be ultimately considered as a manifestation of the quantum spin Hall effect (QSHE) of light [126,65,80].

SOI in scatterer-waveguide systems is not limited to the separation of circular polarizations, as considered in previous experiments $[66,69,77,137,138]$. Indeed, careful engineering of the scatterer enables sorting linearly polarized photons as long as the incident radiation induces a spinning field in it [70]. In a broad sense, SOI in systems showing spin-momentum locking allows mapping the polarization of a light beam into different amplitudes of guided waves propagating along different optical paths or modes. This should enable the recovery of the state of polarization (SoP) of the incoming wave, which is fully described by the Stokes vector $\mathbf{S}=\left(S_{0}, S_{1}, S_{2}, S_{3}\right)^{\mathrm{T}}$, as long as a sufficient number of optical paths are measured. Since the scatterer can be much smaller than the wavelength (ultimately a single atom [139]), SoP would be measured at a local level, thus allowing for detecting the SoP in nanoscale systems as well as in macro-scale wave fronts with spatially-variant polarization $[140,141]$. Therefore, SOI may become a key tool in polarimetry at the nanoscale by enabling extreme shrinking -with all its inherent advantages [110] - of polarimeters (nanopolarimetry). In this chapter, a method for Stokes nanopolarimetry making use of the SOI of light is introduced and experimentally demonstrated at telecom wavelengths on a silicon chip.

\subsection{Description of the concept}

SOI Stokes nanopolarimetry relies on placing a subwavelength scatterer in the close proximity of one or more multimode waveguides, being the system illuminated from the top by a transverse light beam (see

Figure 1.3). In general, each waveguide port may support several guided modes, forming a total of $N$ port-mode combinations, each of them having different SOI behavior associated to the QSHE [60]. Crucially, SOI allows the power excited onto each port-mode combination to be different according to the incident SoP.

As shown in Figure 5.1, the scatterer-waveguide system performing as a Stokes nanopolarimeter is normally illuminated by a monochromatic light beam whose electric field may be generally written as [59]: 


$$
\mathcal{E}^{i n c}(\vec{r})=\left(E_{x}^{i n c}(x, y) \hat{x}+E_{y}^{i n c}(x, y) \hat{y}\right) \exp (-i k z)=A \frac{(\hat{x}+m \hat{y})}{\sqrt{1+|m|^{2}}} \exp \left(-i k_{0} z\right)
$$

where $A(x, y)$ is the wave amplitude at every point, $k_{0}$ is the wave number and the complex number $m(x, y)$ determines the SoP (which, remarkably, may vary across the beam).

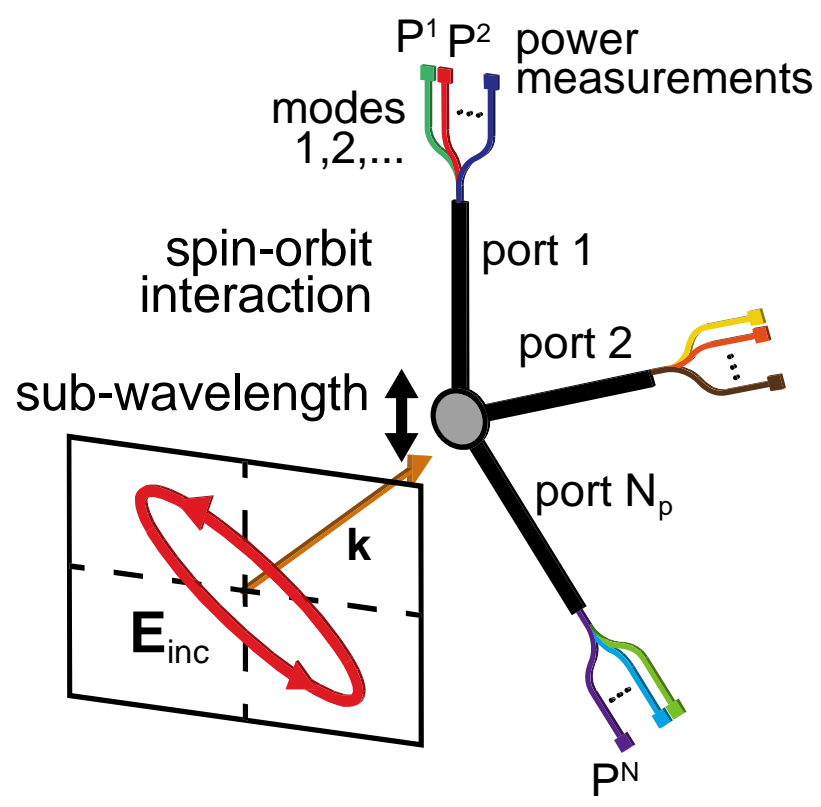

Figure 5.1 A subwavelength scatterer illuminated by a transverse wave will scatter light into different modes of a set of waveguides. SOI allows the scattered power to depend on the input SoP, so measuring the output power for each mode will allow for complete reconstruction the input polarization.

Indeed, the parameter $m$ is related to all the ideal polarizations used to define the Stokes parameters. In the considered coordinate system, $m=$ 0 in horizontal linear polarization $\left(I_{\mathrm{H}}\right), m=\infty$ in vertical linear polarization $\left(I_{\mathrm{V}}\right), m=1$ in $45^{\circ}$ linear polarization $\left(I_{45}\right), m=-1$ in $135^{\circ}$ linear polarization $\left(I_{135}\right), m=i$ in left-handed circular polarization $\left(I_{\mathrm{L}}\right)$, and $m=-i$ in right-handed circular polarization $\left(I_{\mathrm{R}}\right)$. Notice that here it is assumed that the input wave is purely transversal and it does not have longitudinal components of the electric field (this approximation is valid for non-tightly focused beams). In case that the input wave had longitudinal field component, this could not be measured by the 
polarimeter. The beam will impinge on the subwavelength scatterer (notice that the scatterer could be in principle as small as a single atom), so it can be considered locally as a plane wave with a certain power density $I^{\text {inc }}=|A|^{2} / 2 \eta$, where $\eta$ is the medium impedance, and a fixed polarization identified through its normalized Jones vector as follows:

$$
\widehat{\boldsymbol{E}}^{i n c}=\left(\begin{array}{l}
\hat{E}_{x}^{i n c} \\
\widehat{E}_{y}^{i n c}
\end{array}\right)=\frac{1}{\sqrt{1+|m|^{2}}}\left(\begin{array}{c}
1 \\
m
\end{array}\right)
$$

In the general case, the incident wave defined by Equation (5.2) impinges on a very small scatterer coupled to several waveguides or ports, where each waveguide may support a variable number of electromagnetic modes (see Figure 5.1). Notice that, in this scheme, a single straight waveguide in the proximity of the scatterer has two propagation directions, and therefore, two associated ports. It is in principle possible to measure individually the optical power excited in each port-mode combination, which is called $P_{k}$ for the $k$-th port-mode combination. $P_{k}$ will be proportional to the incident power density $I_{\text {inc }}$ on the scatterer, with a proportionality constant termed the effective area, so the scatterer can be considered to operate as an optical antenna. Thanks to SOI, the effective area for the $k$-th port-mode combination will depend on the incident polarization such that:

$$
P_{k}=I_{\text {inc }} A_{\text {eff }}^{k}(m)
$$

The SOI-related dependence of the effective area on the SoP can be completely described by studying the amplitude and phase of the mode excitation under incidence of two orthogonal polarizations. For simplicity, it is assumed horizontal $\mathrm{H}$ (along $x$ ) and vertical $\mathrm{V}$ (along $y$ ) linear polarizations. For $\mathrm{H}$ incidence, the amplitude and phase of the mode that is excited at the $k$-th port-mode combination can be characterized by a complex number $l_{H}^{k}$, such that the power excited is $P_{k}=I_{\text {inc }}\left|l_{H}^{k}\right|^{2}$. Similarly, $P_{k}=I_{\text {inc }}\left|l_{V}^{k}\right|^{2}$ for V incidence. $l_{H}^{k}$ and $l_{V}^{k}$ are the complex effective lengths for the $k$-th port-mode combination, being these complex amplitudes closely related to the scattering-parameter matrix commonly used in engineering. In general, any incident polarization is a linear superposition of the two orthogonal states, so the excited power can be obtained by linear superposition (taking into account the phase) as:

$$
P_{k}=I_{i n c}\left|\hat{E}_{x}^{i n c} l_{H}^{k}+\hat{E}_{y}^{i n c} l_{V}^{k}\right|^{2}
$$


Comparing (5.3) with (5.4) allows us to write the effective area as the square of a dot product between a normalized Jones vector and a vector with the complex effective lengths:

$$
A_{e f f}^{k}(m)=\left|\left(\begin{array}{c}
\hat{E}_{x}^{i n c} \\
\hat{E}_{y}^{i n c}
\end{array}\right) \cdot\left(\begin{array}{c}
l_{H}^{k} \\
l_{V}^{k}
\end{array}\right)\right|^{2} \equiv\left|\widehat{\boldsymbol{E}}^{i n c} \cdot \boldsymbol{l}^{k}\right|^{2}
$$

A dot product is maximum whenever the two vectors are complex conjugates of one another. This motivates to introduce definitions that allow to re-write (5.3) and (5.5) into an intuitive form:

$$
\left\{\begin{array}{c}
P_{k}=I_{\text {inc }} A_{\text {max }}^{k} \overbrace{\left|\widehat{\boldsymbol{E}}^{i n c} \cdot\left(\widehat{\boldsymbol{E}}_{\text {max }}^{k}\right)^{*}\right|^{2}}^{\in[0,1]} \\
A_{\text {max }}^{k} \equiv\left|\boldsymbol{l}^{k}\right|^{2} \\
\widehat{\boldsymbol{E}}_{\text {max }}^{k} \equiv \frac{1}{\left|\boldsymbol{l}^{k}\right|}\left(\boldsymbol{l}^{k}\right)^{*}
\end{array},\right.
$$

where $\hat{\mathbf{E}}_{\text {max }}^{k}$ corresponds to the incident polarization that maximizes the power output on the $k$-th mode: $\max \left(P_{k}\right)=I_{\text {inc }} A_{\text {max }}^{k}$.

It is crucial to realize that, thanks to SOI, the polarization $\hat{\mathbf{E}}_{\text {max }}^{k}$ can be different for each port-mode combination (this requires breaking the mirror symmetry in the system, as discussed later), so that each $k$-th power measurement will be proportional to the geometric projection of the incident polarization Jones vector to the polarization vector $\widehat{\mathbf{E}}_{\max }^{k}$.

So, taking the effective area of the scatterer $A_{\mathrm{eff}}^{k}(\mathbf{E})$ as the power scattered into the $k$-th port-mode combination, divided by the intensity of the incident illumination with polarization $\mathbf{E}$. The power on each port-mode combination is measured and collected in a real vector $\mathbf{P}$, so is important to consider how the power $P_{k}$ varies across the Poincare sphere of incident polarizations. In general, the Stokes vector $\mathbf{S}(\mathbf{E})$ associated to any polarization Jones vector $\mathbf{E}$ is given by:

$$
\boldsymbol{S}(\boldsymbol{E})=\left(\begin{array}{c}
S_{0} \\
S_{1} \\
S_{2} \\
S_{3}
\end{array}\right)=\left(\begin{array}{c}
I_{H}+I_{V} \\
I_{H}-I_{V} \\
I_{45}-I_{135} \\
I_{L}-I_{R}
\end{array}\right)=\left(\begin{array}{c}
\left|E_{x}\right|^{2}+\left|E_{y}\right|^{2} \\
\left|E_{x}\right|^{2}-\left|E_{y}\right|^{2} \\
2 \operatorname{Re}\left(E_{x} E_{y}^{*}\right) \\
-2 \operatorname{Im}\left(E_{x} E_{y}^{*}\right)
\end{array}\right)
$$


Any fully polarized field can be mapped into the surface of the Poincare unit sphere with the coordinates of the normalized 3D Stokes vector:

$$
\hat{\boldsymbol{s}}(\boldsymbol{E})=\frac{1}{S_{0}}\left(\begin{array}{l}
S_{1} \\
S_{2} \\
S_{3}
\end{array}\right)
$$

Starting from these definitions, with some algebra it can be verified that there is a mathematical relation between the squared dot product of any two Jones vectors and the dot product of the corresponding Stokes vectors:

$$
\left|\boldsymbol{E}_{a} \cdot\left(\boldsymbol{E}_{b}\right)^{*}\right|^{2}=\frac{1}{2}\left[\boldsymbol{S}\left(\boldsymbol{E}_{a}\right) \cdot \boldsymbol{S}\left(\boldsymbol{E}_{b}\right)\right],
$$

and with further algebra (realizing that when light is totally polarized $|\mathbf{E}|^{2}=S_{0}^{2}=S_{1}^{2}+S_{2}^{2}+S_{3}^{2}$ ), it can be obtained a corresponding relation between the dot product of the Jones vectors and the angle $\theta$ subtended at the origin of the Poincaré sphere by the two vectors $\widehat{\mathbf{s}}\left(\mathbf{E}_{i}\right)$ :

$$
\begin{aligned}
\left|\boldsymbol{E}_{a} \cdot\left(\boldsymbol{E}_{b}\right)^{*}\right|^{2} & =\left|\boldsymbol{E}_{a}\right|^{2}\left|\boldsymbol{E}_{b}\right|^{2}\left[\frac{1}{2}\left(\hat{\boldsymbol{s}}\left(\boldsymbol{E}_{a}\right) \cdot \hat{\boldsymbol{s}}\left(\boldsymbol{E}_{b}\right)+1\right)\right] \\
& =\left|\boldsymbol{E}_{a}\right|^{2}\left|\boldsymbol{E}_{b}\right|^{2} \cos ^{2}(\theta / 2),
\end{aligned}
$$

Equations (5.9) and (5.10) are general mathematical identities relating arbitrary polarizations and their Stokes vectors. Using them, the power at the $k$-th port-mode combination can be re-written in terms of the Stokes vectors and in terms of the angle between polarizations in the Poincaré sphere:

$$
\begin{aligned}
P_{k} & =I_{i n c} A_{\max }^{k}\left|\widehat{\boldsymbol{E}}^{i n c} \cdot\left(\widehat{\boldsymbol{E}}_{\text {max }}^{k}\right)^{*}\right|^{2} \\
& =I_{\text {inc }} A_{\max }^{k} \frac{1}{2}\left[\boldsymbol{S}\left(\widehat{\boldsymbol{E}}_{\max }^{k}\right) \cdot \boldsymbol{S}\left(\widehat{\boldsymbol{E}}^{i n c}\right)\right], \\
& =I_{\text {inc }} \underbrace{A_{\max }^{k} \cos ^{2}\left(\theta_{k} / 2\right)}_{A_{\text {eff }}^{k}(m)},
\end{aligned}
$$

where $\theta_{k}\left(\hat{\mathbf{E}}^{\mathrm{inc}}\right)$ is the angle subtended at the origin of the Poincare sphere between the polarizations $\widehat{\mathbf{E}}^{\text {inc }}$ and $\hat{\mathbf{E}}_{\text {max }}^{k}$. From Equation (5.11) it can be easily calculated the effective area of the $k$-th port-mode combination on the whole surface of the Poincaré sphere, corresponding to every possible SoP of the incident beam. This is shown in Figure 5.2. In principle, every port-mode combination is 
associated with a plot similar to Figure 5.2 at each wavelength, which depends only on the port, mode and wavelength-dependent $\hat{\mathbf{E}}_{\text {max }}^{k}$. Notice that the effective area is maximum for the polarization $\widehat{\mathbf{E}}_{\max }^{k}$ defined in Equation (5.6), and it is exactly zero for the polarization that lies in the antipodal point in the Poincare sphere, which corresponds to $\hat{\mathbf{E}}_{0}^{k}=\left|\mathbf{l}_{k}\right|^{-1}\left(-l_{V}^{k}, l_{H}^{k}\right)^{T}$, such that $A_{\text {eff }}^{k}\left(\hat{\mathbf{E}}_{0}^{k}\right)=0$.

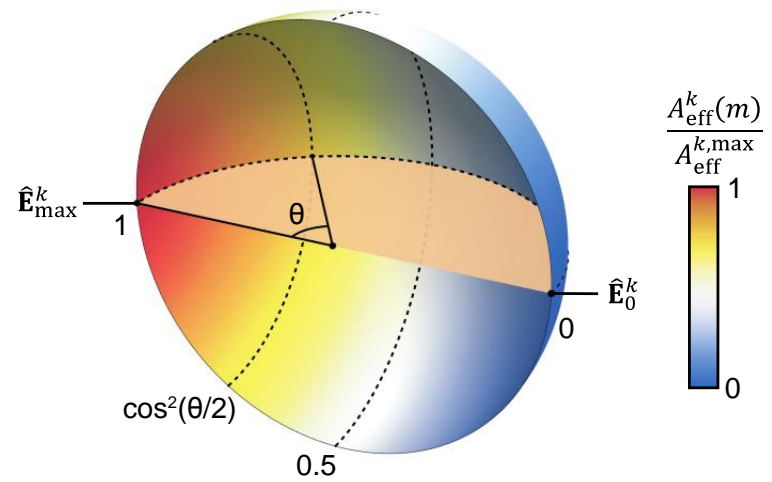

Figure 5.2 Plot of the normalised polarization-dependent effective area as a function of the incident polarization, represented in a cross-section of the Poincaré sphere of incident polarizations.

Equation (5.11) is crucial in this polarimetric approach since it tells that the power output of the $k$-th port-mode combination is a linear combination of the Stokes parameters of incident light polarization. Thus, it constitutes the $k$-th row in a matrix that relates the S-parameters of the incident field (vector $\mathbf{S}\left(\hat{\mathbf{E}}_{\text {inc }}\right)$ of length 4$)$ to the power measured for the outputs $k=1 \ldots N$ (vector $\mathbf{P}$ of length $\mathrm{N}$ ):

$$
\boldsymbol{P}=I_{i n c} \boldsymbol{W} \boldsymbol{S}\left(\widehat{\boldsymbol{E}}_{\text {inc }}\right),
$$

where, from Equation (5.11), the $k$-th row in the polarimetric (or instrument) matrix $\mathbf{W}$ is given by:

$$
\boldsymbol{a}_{k}=\frac{1}{2} A_{\max }^{k} \boldsymbol{S}\left(\widehat{\boldsymbol{E}}_{\max }^{k}\right)^{T}
$$


Notice that, from the definitions in (5.6) and (5.7), this vector can be re-written as $\mathbf{a}_{k}=\frac{1}{2} \mathbf{S}\left(\mathbf{l}^{k^{*}}\right)^{T}$, which applying the definition of Stokes parameters from (5.7) yields:

$$
\boldsymbol{a}_{k}=\frac{1}{2}\left(\left|l_{H}^{k}\right|^{2}+\left|l_{V}^{k}\right|^{2} \quad\left|l_{H}^{k}\right|^{2}-\left|l_{V}^{k}\right|^{2} \quad\left|l_{45}^{k}\right|^{2}-\left|l_{135}^{k}\right|^{2} \quad\left|l_{R}^{k}\right|^{2}-\left|l_{L}^{k}\right|^{2}\right)
$$

where it is defined $l_{45}^{k}=\left(l_{H}^{k}+l_{V}^{k}\right) / \sqrt{2}, l_{135}^{k}=\left(l_{H}^{k}-l_{V}^{k}\right) / \sqrt{2}, l_{R}^{k}=$ $\left(l_{H}^{k}-i l_{V}^{k}\right) / \sqrt{2}$ and $l_{L}^{k}=\left(l_{H}^{k}+i l_{V}^{k}\right) / \sqrt{2}$, which correspond to the complex amplitude of the mode excited under illumination of the corresponding polarizations. Notice that the sign of $S_{3}$ was reversed due to the complex conjugation of $\mathbf{l}^{k}$. From (5.4) this can be re-written in terms of the effective areas of the scatterer for the pure polarizations associated with the different $m$ parameters:

$\boldsymbol{a}_{k}=\frac{1}{2}\left(A_{e f f}^{k}(0)+A_{e f f}^{k}(\infty) \quad A_{e f f}^{k}(0)-A_{e f f}^{k}(\infty) \quad A_{e f f}^{k}(1)-A_{e f f}^{k}(-1) \quad A_{e f f}^{k}(-i)-A_{e f f}^{k}(i)\right)$

In order to use the polarimeter to calculate the incident Stokes vector from the measured powers, i.e. inverting the matrix equation (5.12), a non-singular matrix $\mathbf{W}$ is needed, with at least four linearly independent rows [142]. So, by injecting a proper set of input polarizations (calibration process), the polarimetric matrix $\mathbf{W}$ can be easily formed so that $\mathbf{P}=\mathbf{W} \cdot \mathbf{S}[142]$. Then, the full SoP of any input polarization cold be retrieved as $\mathbf{S}=\overline{\mathbf{W}}^{-1} \cdot \mathbf{P}$ as long as $\mathrm{N} \geq 4$, so the device performs as a Stokes nanopolarimeter.

\subsection{Numerical results}

As an example of the simplest implementation, the scatterer is coupled to a single waveguide with rectangular cross-section $(220 \mathrm{~nm}$ thickness and different widths), being the silicon core completely surrounded by silica. For a silicon core width of $360 \mathrm{~nm}$ (as in the case of the optimal nanopolarimeter detailed later) only two guided modes propagate in the wavelength region around $\lambda=1,550 \mathrm{~nm}$, as depicted in Figure 5.3. Such modes are typically used in integrated silicon photonics circuitry: the fundamental even or TE-like mode (characterized by a main electric 
field component along $y$ ), and the fundamental odd or TM-like mode (characterized by a main electric field component along $z$ ). The crosssections of the three electric field components for the two fundamental guided modes considering propagation along positive values of the $x$ axis (forward or + propagation) are shown in Figure 5.4 at $\lambda=1550 \mathrm{~nm}$. It has been checked that the different field components display a similar distribution - remarkably, the symmetries with respect to the main axes remain identical - for other core dimensions as well as at other wavelengths.

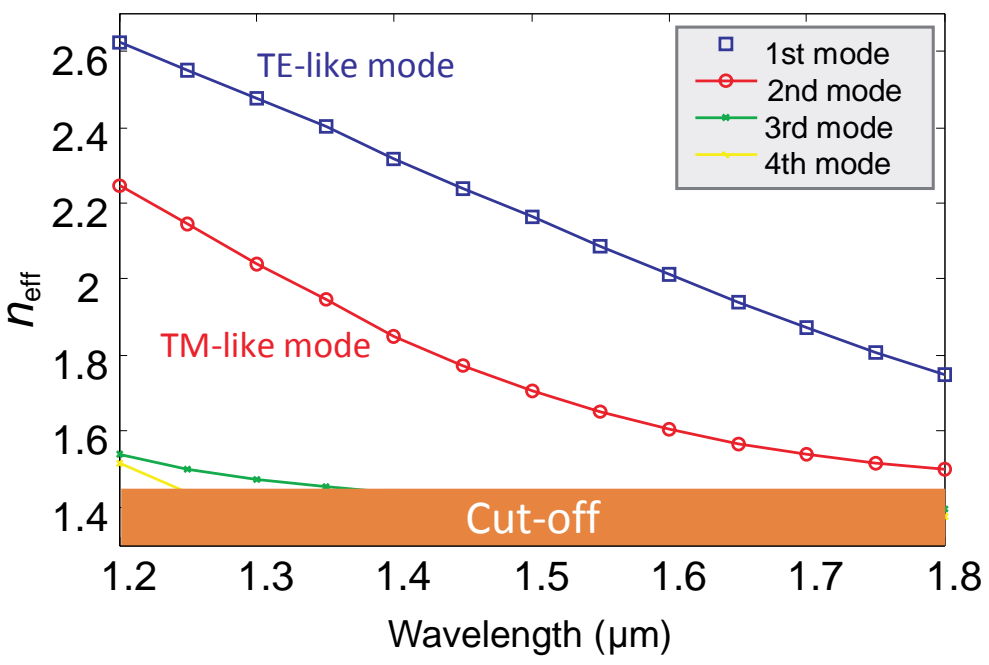

Figure 5.3 Effective index $\left(\mathrm{n}_{\mathrm{eff}}\right)$ of the guided modes of a silicon waveguide with $220 \times 360 \mathrm{~nm}$ cross section on a silica substrate in the wavelength region between 1.2 and $1.8 \mu \mathrm{m}$.

Moreover, such field profiles are also retained for an asymmetric configuration in which the waveguide core is not up-covered with silica. A remarkable longitudinal field component responsible for transverse spin as well as SOI-related phenomena is observed in all the cases, both outside (evanescent field) and inside the waveguide silicon core (as seen in Chapter 2). Notice that this component of the electric field $\left(E_{\mathrm{x}}\right)$ will reverse its sign for backward propagation (towards $-\mathrm{x}$ ), which is a clear signature of the spin-momentum locking or QSHE of light intrinsic to both evanescent and guided waves [65]. The crosssectional maps are distinct for different modes, which ensures encoding of the incident polarization into different optical intensities coupled towards backward and forward directions for each considered mode. 
This enables the realization of distinct measurements on the SoP, as required in polarimeters. Additional guided modes propagate at lower wavelengths as shown in Figure 5.3. Increasing the size of the waveguide core cross-section would allow the appearance of more guided modes at the wavelength region around $1,550 \mathrm{~nm}$. All such guided modes would also show transverse spin but different field profiles, being useful to perform additional polarimetric measurements. For the simplest case of a single straight waveguide with a coupled scatterer, if the waveguide supports $M$ guided modes (with $M \geq 2$ ), the resulting matrix $\mathbf{W}$ would have dimensions $2 M \times 4$, being $2 M \geq 4$. Of course, the number of measurements for a single SoP (and therefore in a single shot) could be further increased by using more waveguides, which leads us to the general polarimeter scheme shown in Figure 5.1. In general, increasing the number of measurements -via either extra modes or additional waveguides - per input SoP is useful to reduce noise-induced errors in the retrieval of the Stokes parameters [143,144].

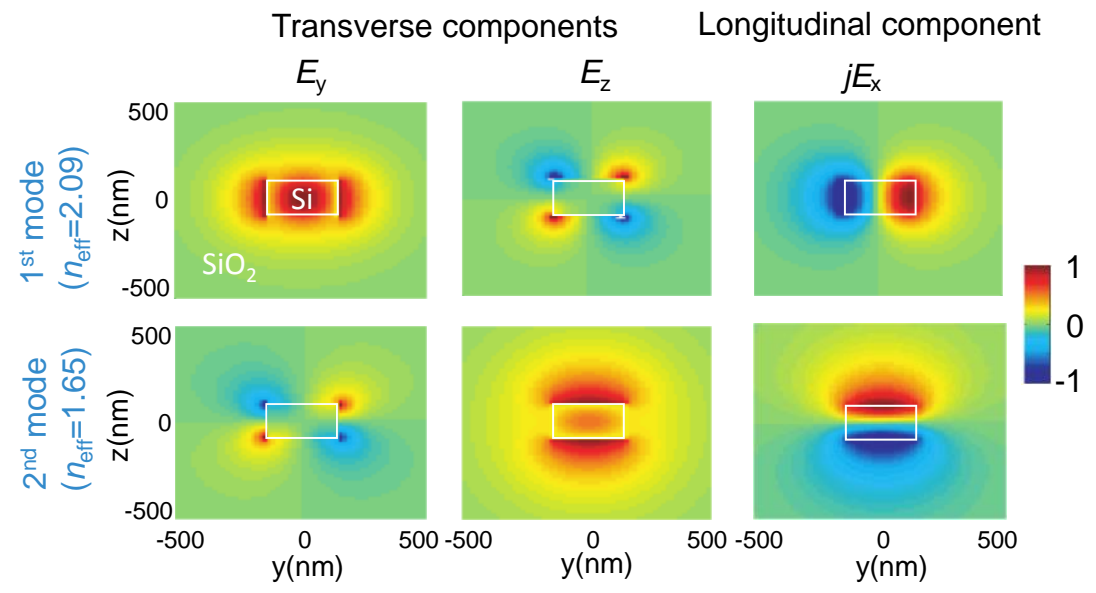

Figure 5.4 Numerically calculated cross-sectional profiles of the electric field components of the first two propagating modes in a silicon waveguide $(360 \mathrm{~nm} \times 220 \mathrm{~nm}$ cross-section) surrounded by silica at $\lambda=1,550 \mathrm{~nm}$. Left panels: $\mathrm{E}_{\mathrm{y}}$ component; Middle panels: $E_{\mathrm{z}}$ component; Right panels: $j \mathrm{E}_{\mathrm{x}}$ component (longitudinal field responsible for transverse spin and SOI related effects). The solid white rectangle highlights the boundary between the silicon core and the surrounding silica cladding.

Thus, the SOI nanopolarimeter may consist in a silicon waveguide designed to support two guided modes (TE-like and TM-like) at the 
operating wavelength and having a lateral protuberance on it. But not any configuration is valid, breaking of symmetry is fundamental for SOI effects to allow the splitting of elliptical polarizations into different waveguide port-mode combinations. If the scatterer-waveguides system is mirror symmetric with respect to a plane that contains the incidence direction, and this plane also contains the propagation direction of one of the output ports, then it follows that the two circular polarizations must excite that port equally, because the mirror symmetry exchanges left-handed and right-handed incident polarizations while leaving the structure unchanged. Therefore, $A_{\mathrm{eff}}^{k}(-i)=A_{\mathrm{eff}}^{k}(i)$ which implies $S_{3}\left(\hat{\mathbf{E}}_{\text {max }}^{k}\right)=0$, so that the polarization $\hat{\mathbf{E}}_{\max }^{k}$ must be linear.

(a)

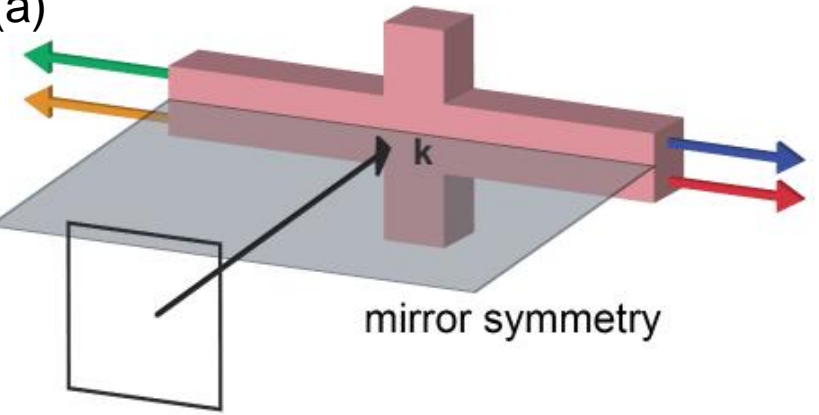

incident plane wave

(b)

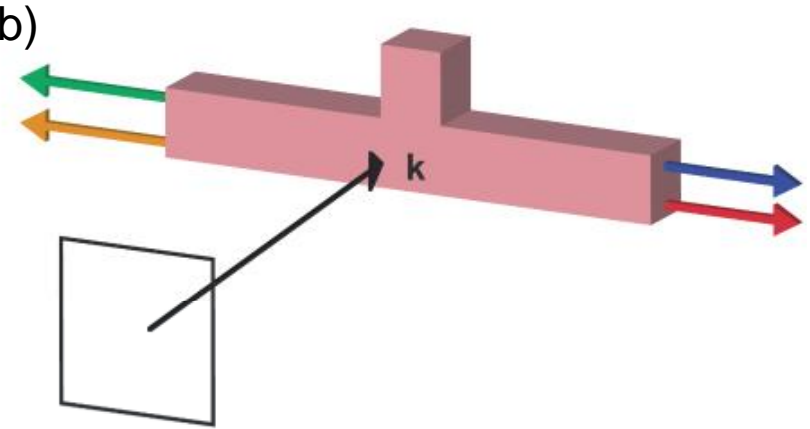

Figure 5.5 (a) Structure with mirror-symmetry on a plane containing the ports will not work as a polarimeter. All outputs in the ports will have a linearly polarized $\hat{\mathbf{E}}_{\max }^{\mathrm{k}}$. (b) Breaking the mirror symmetry allows the split of non-parallel polarizations into the four port-mode combinations, enabling its use as a Stokes polarimeter. 
In fact, symmetry considerations show that this linear polarization will be either parallel or perpendicular to the plane of symmetry containing the port, depending on the port mode symmetry. If two ports are contained in the same mirror symmetry plane, they will both share the same linear polarization $\hat{\mathbf{E}}_{\text {max }}^{k}$ for the same modes (except for at most a time phase difference), and thus will not result in independent rows in the polarimetric matrix. In the simplest case studied in this section (a straight waveguide with two ports with two guided modes each) breaking mirror symmetry with respect to the plane containing each port propagation direction is required to split four different input polarizations into each port-mode combination. For this reason, Figure 5.5(a) would not work as polarimeter, but Figure 5.5(b) would.

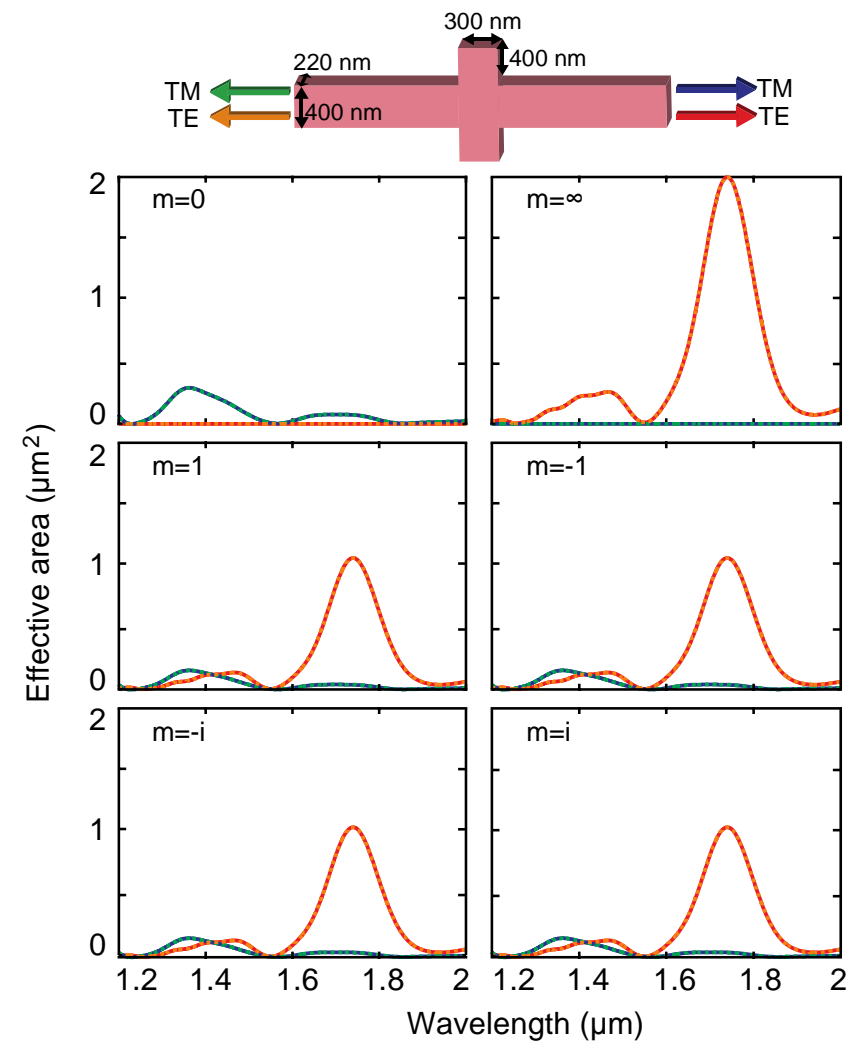

Figure 5.6 Effective areas of the four port-mode combinations for different input polarizations $(\mathrm{m}=0, \infty, 1,-1, \mathrm{i},-\mathrm{i})$ as a function of the wavelength for mirror symmetric configuration. 
From the point of view of SOI, it could be stated that the effects produced by each scatterer (each one placed in a region having longitudinal field component of opposite signs) in the symmetric configuration would cancel each other, thus avoiding spin-controlled directional guiding phenomena. This finding is verified by numerically calculating (simulation details in Methods) the effective areas resulting from different input polarizations for both structures, as shown in Figure 5.6.It can be seen that when the scatterer-waveguide system displays mirror symmetry the effective areas for the polarization pairs $(m=1, m=-1)$ and $(m=i, m=-i)$ are identical for each mode, which would result in a non-invertible matrix $\mathbf{W}$, that can be attributed to the cancellation of the SOI effects.

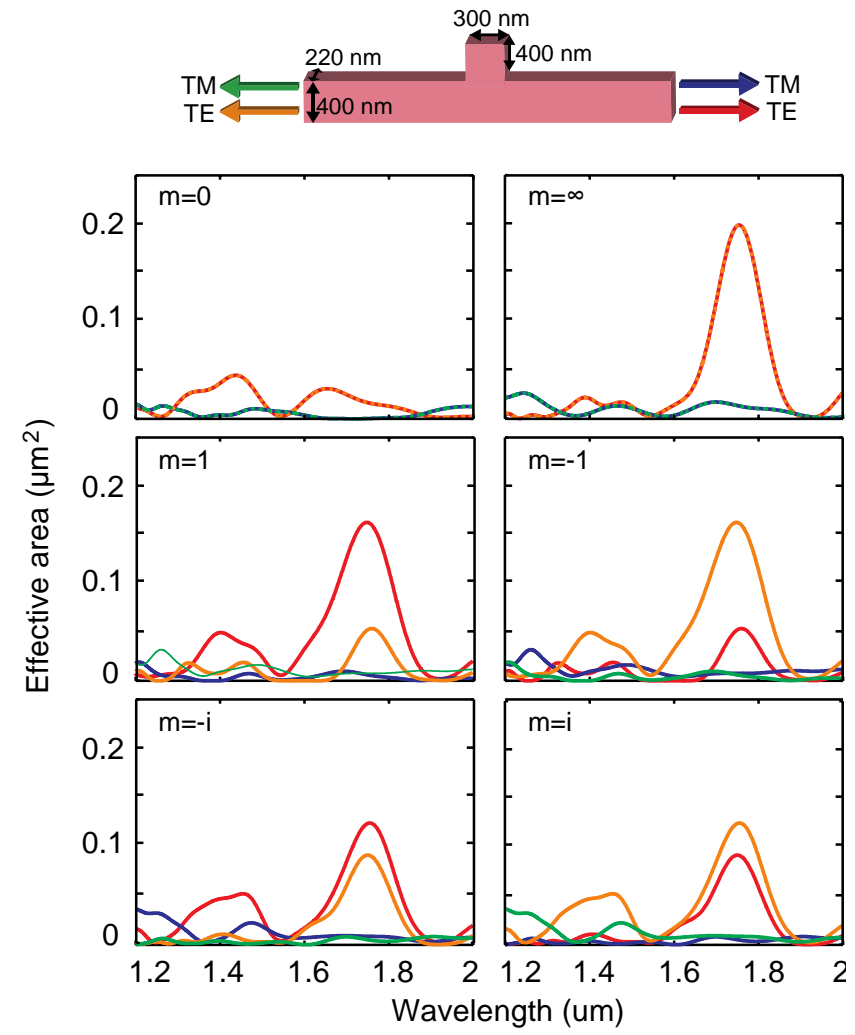

Figure 5.7 Effective areas of the four port-mode combinations for different input polarizations $(\mathrm{m}=0, \infty, 1,-1, \mathrm{i},-\mathrm{i})$ as a function of the wavelength after breaking mirror symmetry. 
The situation changes when the mirror symmetry is broken (see Figure 5.7) so that SOI produces that the obtained effective areas are different for each output-mode combination.

As demonstrated previously, there exists an incident polarization $\mathbf{E}_{\max }^{k}$ for each port-mode combination $k$ that maximizes the corresponding effective area $A_{\text {eff }}^{k}\left(\mathbf{E}_{\max }\right)=A_{\text {max }}^{k}$. Figure 5.8 shows the four numerically calculated polarization ellipses corresponding to $\mathbf{E}_{\max }^{T E+}$, $\mathbf{E}_{\max }^{T E-}, \mathbf{E}_{\max }^{T M+}$ and $\mathbf{E}_{\max }^{T M-}$, which maximize the effective area of the TE and TM-like modes propagating on the $+\mathrm{x}$ or $-\mathrm{x}$ direction on the designed structure, for the specific T-block scatterer at a wavelength $\lambda=1,569 \mathrm{~nm}$.

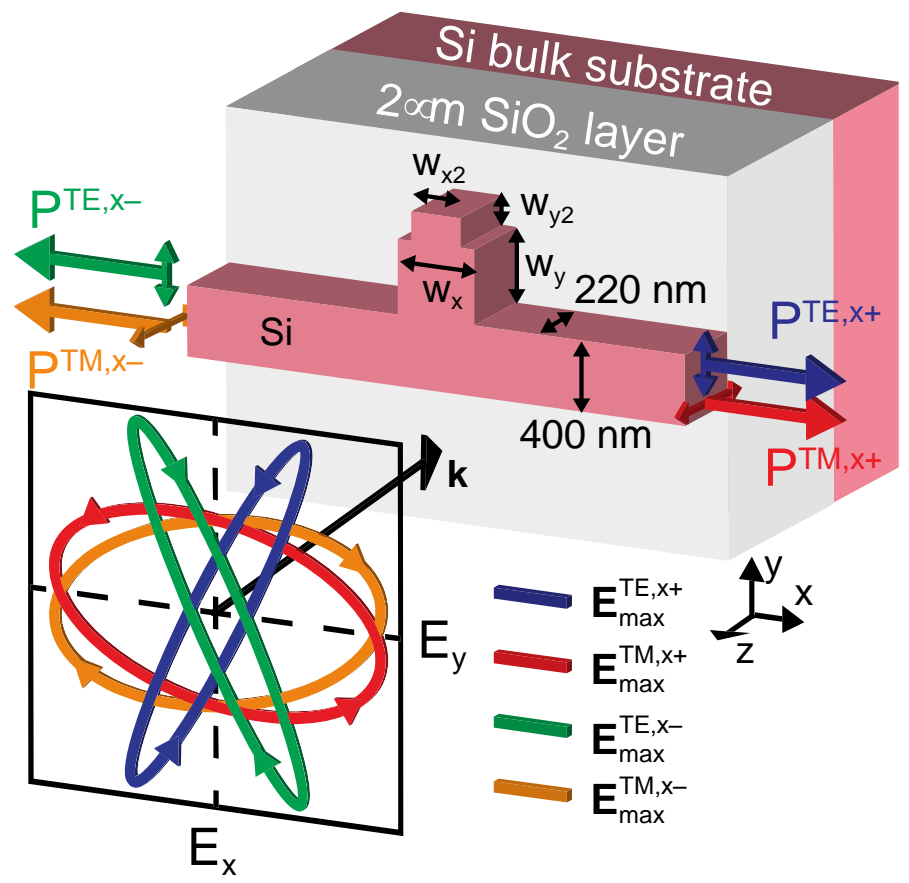

Figure 5.8 In its simplest form, a SOI Stokes nanopolarimeter will consist on a bimodal silicon waveguide (supporting TE-like and TM-like guided modes) laterally perturbed by a scatterer, which breaks the mirror symmetry and enables SOI. The input polarizations providing maximum output power for the four different port-mode combinations at a wavelength $\lambda=1,569 \mathrm{~nm}$ are depicted. This T-block shaped scatterer provides an optimal polarimetric response. 
When the incident beam carries any one of these polarizations, the amplitude of one of the four components of the measured powers $\mathbf{P}$ will be maximum. Thus, $\mathbf{P}$ carries information about the relative amplitude of these four polarization components, from which the original SoP can be reconstructed as explained before.

As long as the mirror symmetry of the scatterer-waveguide system with respect to the $x y$ and $x z$ planes is broken, SOI will make $\mathbf{W}$ invertible. However, bad conditioning of $\mathbf{W}$ may lead to inaccurate results mainly in the context of faint scattered signals that could be obscured by undesired noise, as it usually happens in nanoscale optical signals.

\subsubsection{Optimization of the nanopolarimeter}

Remarkably, the SOI nanopolarimeter can be easily designed to perform optimally, in the sense of minimizing the condition number of W. In the simplest structure considered in this section (Figure 5.8), there is a straight silicon waveguide with two ports $\left(\mathrm{x}_{+}\right.$and $\left.\mathrm{x}_{-}\right)$ supporting two guided modes each (TE and TM), so all four rows, corresponding to the Stokes vector of $\mathbf{l}^{k^{*}}$ for the two directions and two modes, need to be independent. This means that the four polarizations $\hat{\mathbf{E}}_{\max }^{k}$ must be different, which can be achieved by breaking the mirror symmetry, as shown in Figure 5.5 and Figure 5.6. Therefore, any kind of asymmetry with respect to the xz plane will be sufficient to give an invertible matrix $\mathbf{W}$. This means that that there is an infinite number of possible scatterer-waveguide systems that work as a polarimeter, so in this sense, the system described here is extremely robust against fabrication inaccuracies or disorder. However, the performance of all these polarimeters will be different. It is intuitive to see that, for the measurement to be as robust as possible with respect to noise in the measurement, firstly the effective areas need to be as big as possible, and secondly the different polarizations $\hat{\mathbf{E}}_{\text {max }}^{k}$ need to be as different as possible. Mathematically this second criterion is formally expressed by requiring the matrix $\mathbf{W}^{-1}$ to have the smallest possible condition number $\kappa$, defined by:

$$
\kappa(\boldsymbol{W})=\left\|\boldsymbol{W}^{-1}\right\|_{2}\|\boldsymbol{W}\|_{2}
$$

where $\|\cdot\|_{2}$ stands for the Euclidean norm. For the polarimetric matrix of a generic polarimeter, the minimum condition number is $(M-1)^{1 / 2}$, where M corresponds to the Stokes vector dimension [145]. In the special case that the four maximum effective areas for the four outputs 
$A_{\text {max }}^{k}$ are equal, the minimization of $\kappa(\mathbf{W})$ depends exclusively on the polarizations sorted by each output $\hat{\mathbf{E}}_{\text {max }}^{k}$. For these polarizations to be as different as possible, and thus achieve a minimum condition number, it was shown that the volume occupied by the tetrahedron whose vertices are the four $\hat{\boldsymbol{s}}\left(\hat{\mathbf{E}}_{\text {max }}^{k}\right)$ on the Poincaré sphere has to be maximum $[128,132]$. This is achieved when the inscribed tetrahedron is a regular tetrahedron.

In order to find the optimal SOI polarimeter, it is necessary to design the four $\hat{\mathbf{E}}_{\text {max }}^{k}$ to be the vertices of a regular tetrahedron in the Poincare sphere while having the same effective area $A_{\text {max }}^{k}$. For simplicity, the structure will be $x=0$ mirror symmetric, which means that the polarizations $\hat{\mathbf{E}}_{\text {max }}^{k}$ associated to opposite ports for a given mode (TM or TE) must follow that same mirror symmetry. An $x=0$ mirror symmetry in polarizations is equivalent to changing the sign of Stokes parameters $S_{2}$ and $S_{3}$ while keeping the sign of $S_{1}$. Notice that, in contrast, the metasurface polarimeter in [132] only reverses the sign of $\mathrm{S}_{3}$, which impedes the formation of a tetrahedron with non-zero volume inside the Poincaré sphere. This introduces a restriction in the design, by making the pair of polarizations associated with TE waveguide modes $\hat{\mathbf{E}}_{\max }^{T E, x+}, \hat{\mathbf{E}}_{\max }^{T E, x-}$ propagating in opposite directions to be necessarily mirror symmetric of each other and thus show $180^{\circ}$ rotation symmetry around axis $S_{1}$ in the Poincare sphere. The two TM waveguide outputs $\hat{\mathbf{E}}_{\max }^{T M, x+}, \hat{\mathbf{E}}_{\max }^{T M, x-}$ will also show an identical relation. Given this restriction, the optimal polarizations must generate a regular tetrahedron in the Poincaré sphere that itself fulfills the same $180^{\circ}$ rotation symmetry around axis $S_{1}$. The solutions that fulfil this condition for optimality are given by:

$$
\begin{gathered}
\hat{\boldsymbol{s}}\left(\widehat{\boldsymbol{E}}_{\text {max }}^{1}\right)=\frac{1}{5}\left(\begin{array}{c}
3 \\
4 \cos (\alpha) \\
4 \sin (\alpha)
\end{array}\right) \quad \leftrightarrow \quad \hat{\boldsymbol{s}}\left(\widehat{\boldsymbol{E}}_{\text {max }}^{2}\right)=\frac{1}{5}\left(\begin{array}{c}
3 \\
-4 \cos (\alpha) \\
-4 \sin (\alpha)
\end{array}\right) \\
\hat{\boldsymbol{s}}\left(\widehat{\boldsymbol{E}}_{\text {max }}^{3}\right)=\frac{1}{5}\left(\begin{array}{c}
3 \\
4 \cos (\alpha+\pi / 2) \\
4 \sin (\alpha+\pi / 2)
\end{array}\right) \leftrightarrow \hat{\boldsymbol{s}}\left(\widehat{\boldsymbol{E}}_{\text {max }}^{4}\right)=\frac{1}{5}\left(\begin{array}{c}
3 \\
-4 \cos (\alpha+\pi / 2) \\
-4 \sin (\alpha+\pi / 2)
\end{array}\right)
\end{gathered}
$$

with the corresponding Jones' vectors: 


$$
\begin{aligned}
& \widehat{\boldsymbol{E}}_{\text {max }}^{1}=\frac{1}{\sqrt{5}}\left(\begin{array}{c}
2 e^{-i \alpha} \\
1
\end{array}\right) \leftrightarrow \widehat{\boldsymbol{E}}_{\text {max }}^{2}=\frac{1}{\sqrt{5}}\left(\begin{array}{c}
-2 e^{-i \alpha} \\
1
\end{array}\right) \\
& \widehat{\boldsymbol{E}}_{\text {max }}^{3}=\frac{1}{\sqrt{5}}\left(\begin{array}{c}
i e^{-i \alpha} \\
2
\end{array}\right) \leftrightarrow \widehat{\boldsymbol{E}}_{\text {max }}^{4}=\frac{1}{\sqrt{5}}\left(\begin{array}{c}
-i e^{-i \alpha} \\
2
\end{array}\right),
\end{aligned}
$$

where the free parameter $\alpha$ represents a rotation of the polarizations around the $S_{1}$ axis in the Poincaré sphere. Figure S6 shows two particular examples of optimal polarizations with $\alpha=0$ and $\alpha=\pi / 4$ respectively. Notice that all four polarizations fulfill $S_{1}= \pm 3 / 5$, with opposite signs associated to the different pairs. There is a freedom to choose the sign for the $S_{1}$ parameter of the TE modes, and the opposite sign must then be designed for the TM modes. The tetrahedron can be rotated freely around the $S_{1}$ axis (parameter $\alpha$ ) while keeping the required symmetry, but it is evident that rotation of the tetrahedron around any other axis would break that symmetry, graphically showing that this is the only solution.
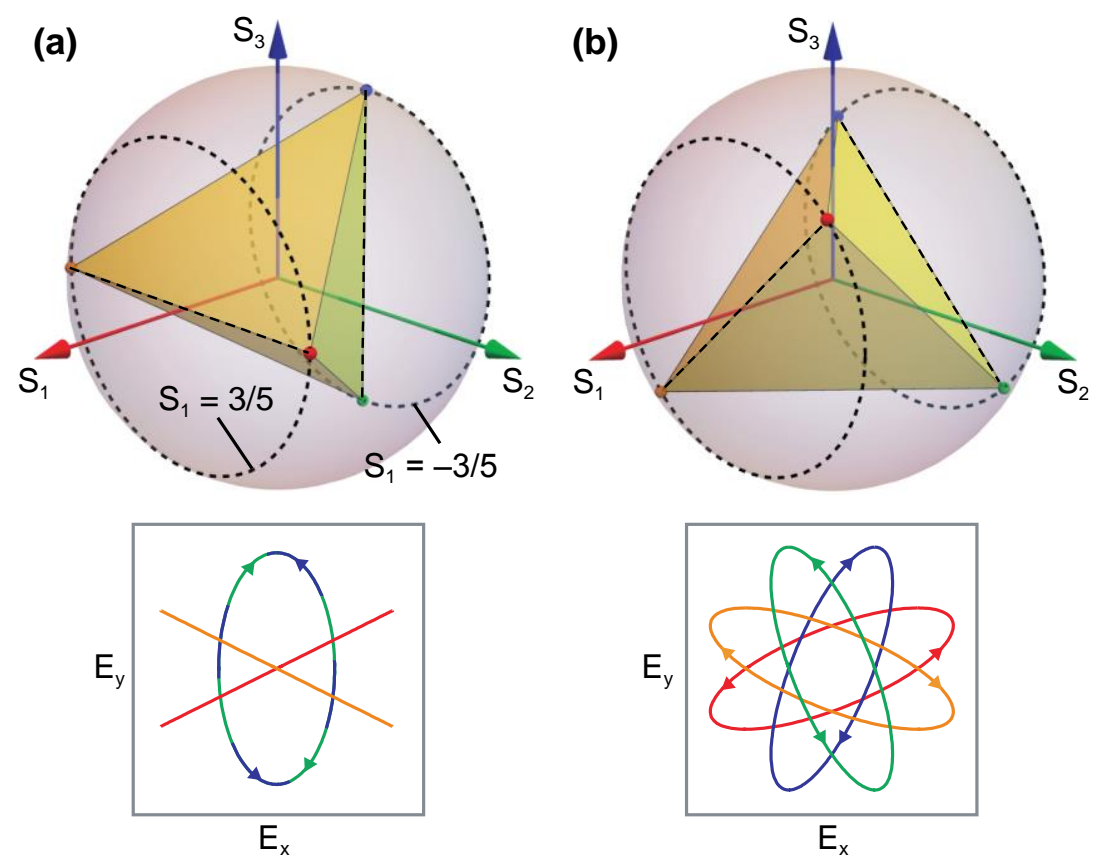

Figure 5.9 Optimal polarizations shown as polarization ellipses and as tetrahedrons in the Poincaré sphere following Equation (5.16) for (A) $\alpha=0$ and (B) $\alpha=\pi / 4$. 
So, for a polarimeter with 4 outputs with equal effective areas $A_{\text {max }}^{k}$, minimizing the condition number of $\mathbf{W}$ is equivalent to maximizing the volume of the tetrahedron inscribed in the Poincare sphere whose vertices are the four SoP's corresponding to the four different $\mathbf{E}_{\max }^{i}$ $[127,128]$. When the scatterer and the two output ports are mirror symmetric, as the "+" and "-" outputs in the structure and many previous SOI experiments, it follows that the effective areas of opposite outputs of the same mode are equal $A_{\text {eff }}^{+}\left(\mathbf{E}_{1}\right)=A_{\text {eff }}^{-}\left(\mathbf{E}_{2}\right)$ whenever $\mathbf{E}_{1}$ and $\mathbf{E}_{2}$ are related by the same mirror symmetry. It is therefore needed to design two polarizations $\mathbf{E}_{\max }^{T E+}$ and $\mathbf{E}_{\max }^{T M+}$ such that, together with their mirror symmetric counterparts $\mathbf{E}_{\max }^{T E-}$ and $\mathbf{E}_{\max }^{T M-}$ form an optimum set of polarizations that maximize the tetrahedron volume. But design an optimal nanopolarimeter requires a smart design of the scatterer-waveguide system in order to accomplish the criteria given by (5.17) and (5.18). In the quest for the optimal polarimeter, the starting point was the structure previously used in Refs. [29,70] - a rectangular protrusion in a silicon waveguide - because of its fabrication simplicity, the absence of metals (which can introduce absorption losses at the expenses of larger effective areas) and, remarkably, its capability to sort out linear-polarized waves at certain polarization angles for the TE modes, which in principle could satisfy (5.17). Since the basic structure (which will be called rectangular-shape scatterer) is not optimal, the socalled simplex optimization method has been used in MATLAB, together with full vectorial simulations performed with CST Microwave Studio. The Simplex method is an iterative algorithm for the minimization of a target function by varying a finite set of parameters. Starting from the value of the target function at a given point, the procedure consists of seeking for another point that improves the previous value. These points are vertexes of a $\mathrm{N}$-dimensional polytope in the parameter space, that constitutes the region determined by the restrictions to which the problem is subject to (the so called feasible region). The search is performed by means of displacements of the edges of the polytope, from the current vertex up to the adjacent one, so that it improves the target value of the function. The optimization consisted of parameterizing the size and shape of the scatterer, converting the rectangular scatterer into a T-block shaped scatterer in order to have more variable parameters. Notice that, in principle, there are many other shapes that could end up in an optimal nanopolarimeter. As explained in the previous section, the SOI polarimeter is considered optimal when the four $\hat{\mathbf{E}}_{\max }^{k}$ describe a regular tetrahedron in the Poincaré sphere and the two maximum effective areas $\mathbf{A}_{\max }^{\mathrm{TM}}$ and $\mathbf{A}_{\max }^{\mathrm{TE}}$ are equal, so the target of the 
optimization was to maximize the volume of the tetrahedron (given by $\widehat{\mathbf{E}}_{\text {max }}^{k}$ ) while keeping equal effective areas. In Figure 5.10(c) it is depicted the evolution of the optimizer along each iteration, starting from the studied rectangular shape scatterer and reaching an optimal Tblock shaped scatterer design having a set of polarization states Figure 5.10(b) that define a tetrahedron with a volume that is $99.7 \%$ of the maximum attainable value and a difference between effective areas of $2.7 \%$.

(a)
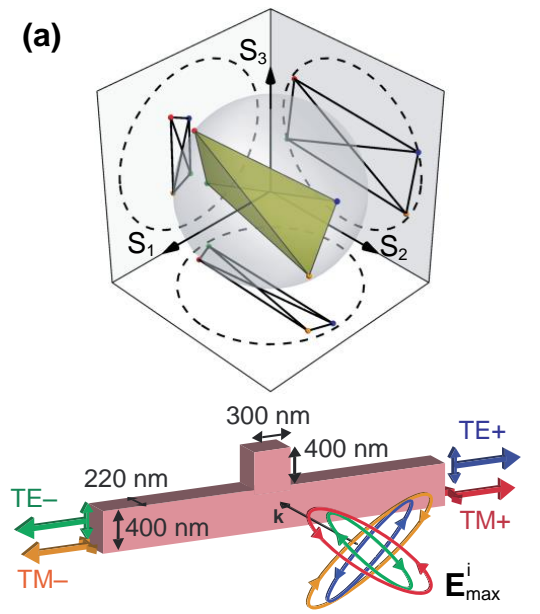

(b)

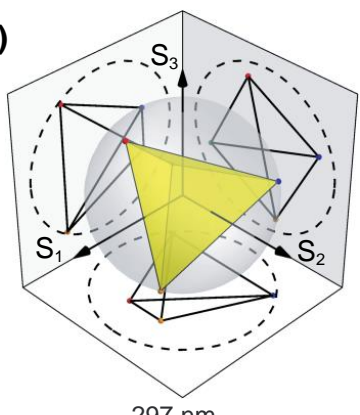

$297 \mathrm{~nm}$

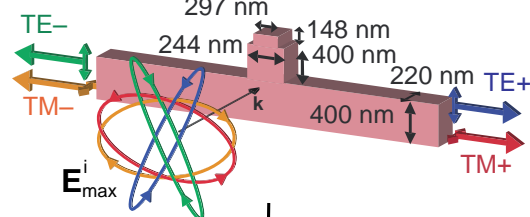

(c)

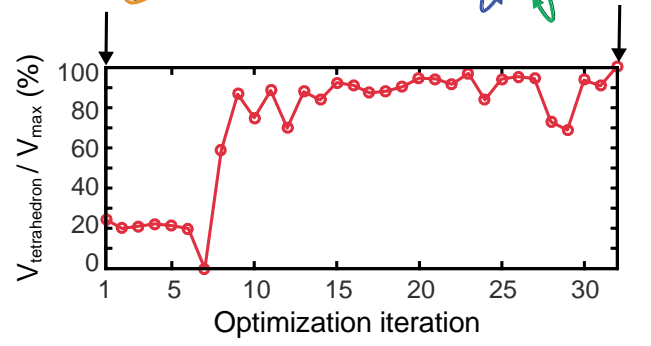

Figure 5.10 Starting from a rectangular scatterer asymmetrically coupled to the silicon waveguide in (a), it is found an optimal Tblock scatterer shown in (b) via an optimization process that maximizes the volume of the tetrahedron inscribed within the Poincaré sphere. The tetrahedrons for each scatterer-waveguide configuration as well as the polarizations giving rise to maximum optical power for the different port-mode pairs are depicted. 


\subsection{Experimental Results}

In order to retrieve the input SoP, it is considered each nanoantena portmode combination $k$ as an independent polarization analyzer. For the case of the waveguide with the single scatterer, making use of the fundamental even and odd modes (TE and TM-like modes) at both output ports, the polarimeter has $Q=4$ polarization analyzers. Thus, it will be able to retrieve the input SoP as long as the polarimetric matrix $\mathbf{W}$ is invertible, which is achieved by breaking the mirror symmetry so that SOI plays a role, as previously discussed. Each polarization analyzer is characterized by an analyzer vector - defined equivalently to a Stokes vector [142] -containing four elements, as defined in Equation (5.15). In the analyzer vector definition the $m$ parameter takes six different values, which correspond to the six "calibration measurements" used to conform experimentally the polarimetric matrix W for each wavelength, which can be written as:

$$
\boldsymbol{W}=\left(\begin{array}{cccc}
A_{T E, x+}(0)+A_{T E, x+}(\infty) & A_{T E, x+}(0)-A_{T E, x+}(\infty) & A_{T E, x+}(1)-A_{T E, x+}(-1) & A_{T E, x+}(-i)-A_{T E, x+}(i) \\
A_{T M, x+}(0)+A_{T M, x+}(\infty) & A_{T M, x+}(0)-A_{T M, x+}(\infty) & A_{T M, x+}(1)-A_{T M, x+}(-1) & A_{T M, x+}(-i)-A_{T M, x+}(i) \\
A_{T E, x-}(0)+A_{T E, x-}(\infty) & A_{T E, x-}(0)-A_{T E, x-}(\infty) & A_{T E, x-}(1)-A_{T E, x-}(-1) & A_{T E, x-}(-i)-A_{T E, x-}(i) \\
A_{T M, x-}(0)+A_{T M, x-}(\infty) & A_{T M, x-}(0)-A_{T M, x-}(\infty) & A_{T M, x-}(1)-A_{T M, x-}(-1) & A_{T M, x-}(-i)-A_{T M, x-}(i)
\end{array}\right)
$$

Where each element $A_{n, x \pm}(m)$ is the effective area of the analyzer provided by the $n$-th guided mode propagating forwards (+) or backwards (-) when the polarimeter is excited by an incoming light with a polarization defined by $m$. After calibration, the incident $\operatorname{SoP}(\mathbf{S})$ will be reconstructed by measuring the optical power at the four outputs $(\mathbf{P})$ for a given wavelength and applying:

$$
\boldsymbol{S}=\boldsymbol{W}^{-1} \boldsymbol{P} / I_{\text {inc }}
$$

Several fabricated Stokes nanopolarimeters were demonstrated at telecom wavelengths. SOI nanopolarimeters were fabricated using standard Si-fabrication tools (see Methods). Besides the active region, the output waveguides were made long enough to carry out the polarization-dependent optical power in each mode to the chip boundary, from which they could be detected by using an infrared camera as shown in Figure 5.11. The measurement set-up is depicted in Figure 5.12. A tunable laser at wavelengths covering the range between 1,320 and $1,630 \mathrm{~nm}$ was used as light source, controlling the polarization at its output with a fiber polarization controller. The optical power generated by the laser was of the order of $1 \mathrm{~mW}$. The 
subwavelength active region of the sample (this is, the region containing the scatterer coupled to the waveguide in which SOI takes place) was illuminated from a cleaved optical fiber with a special final section containing a $2 \mathrm{~mm}$ long quarter wave plate (QWP) provided by Fibercore in order to allow the circularly and elliptically polarized light illumination.

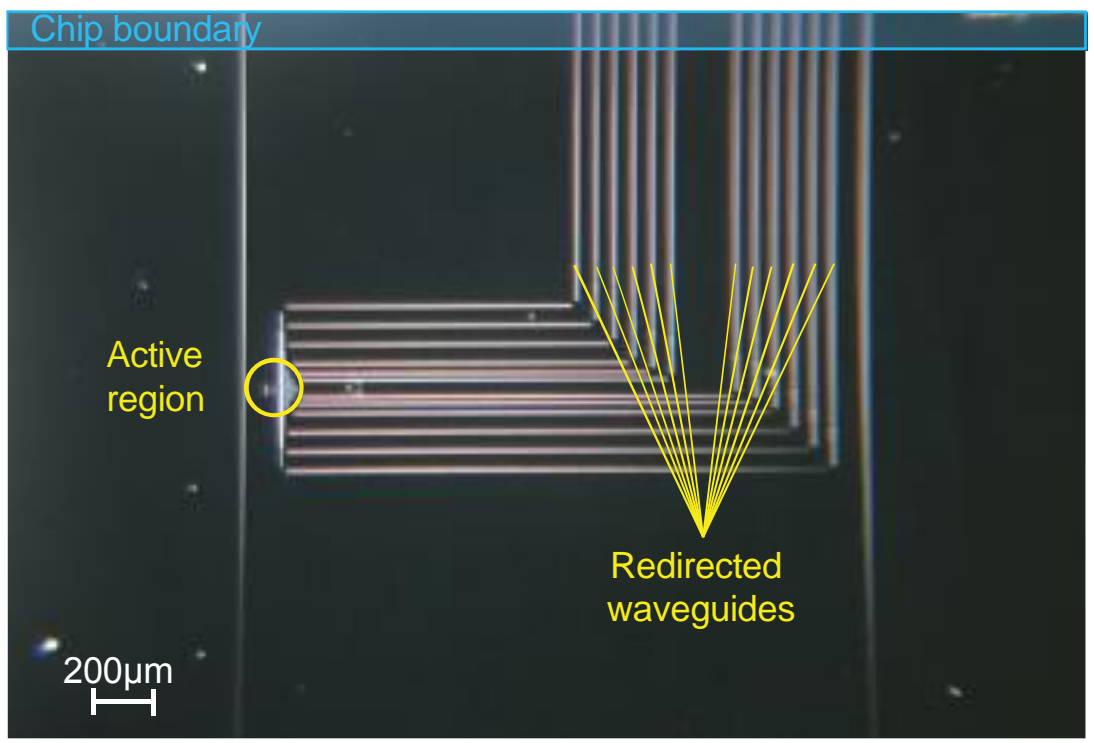

Figure 5.11 Optical microscope image of a fabricated sample. Note that the optical waveguides coming from the active region are redirected to the chip boundary in order to capture them simultaneously with the infrared camera. The scatterers are placed in the so-called active region, where SOI takes place.

The sample was placed vertically (see Figure 5.12), so that two orthogonal paths were created: the "vertical path" is the light scattered by the polarimeter into the integrated waveguides and towards the top edge of the sample, from which the device outputs could be measured. The "horizontal path" corresponds to the illuminating light that propagates through the silicon substrate -transparent at the employed wavelengths- that is used to monitor the incident polarization. In the horizontal path, the polarization was monitored by using an objective for collimating the radiation passing through the silicon substrate and a free-space linear polarizer (FPC). Once the light passed through the FPC a 50:50 splitter was used to split up the power between a power meter (Newport 1930C) and an IR camera (Indigo Alpha NIR). Linear input polarizations were obtained by minimizing the orthogonal 
polarization component reaching an axial ratio $>20 \mathrm{~dB}$. For monitoring circular and elliptical input polarizations, an extra QWP between the objective and the FPC (see Figure 5.12) is used.

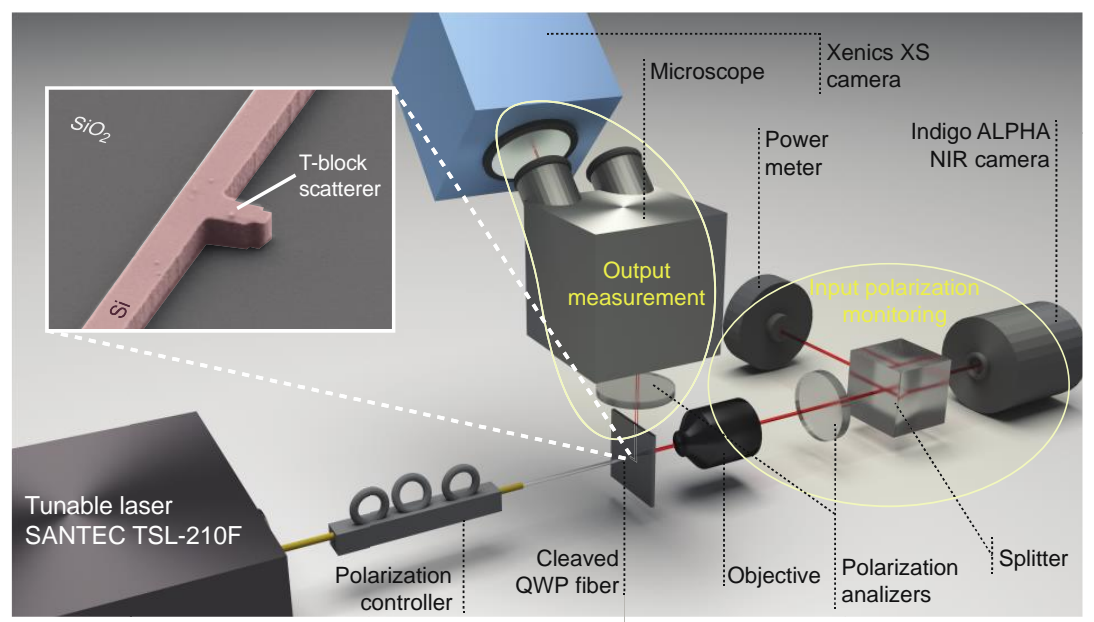

Figure 5.12 Experimental setup. Inset: SEM image of one of the measured samples.

This allowed us to convert such inputs into linear polarization, which was detected. The achievement of purely circular polarizations was checked by removing the QWP afterwards (axial ratios of the order of $0.5 \mathrm{~dB}$ were measured). The vertical path was used to recover the optical output power of the polarimeter waveguides with a IR camera (Xenics XS-XC117) mounted at the position of the eyepiece of a $4 \times$ microscope (National Stereoscopic Microscopes Zoom model 420 series), focused on the waveguide outputs (Fig. Figure 5.13(a)). An FPC placed horizontally between the waveguide outputs and the microscope was required in order to separate the TE and TM-like outputs of the polarimeter. The camera counts were integrated in the scattering spots so as to capture the spot intensity in each output port (Fig. Figure 5.13(b)).

Notice that this experimental set-up is possible because of the nondestructive nature of the polarimetric approach: the light passing through the chip is only slightly depolarized because of the extraction of a tiny amount of power (which will depend on the SoP of the signal) so the polarization can be simultaneously detected in the vertical (via the nanopolarimeter) and the horizontal (via common free-space polarimetric elements) paths. Therefore, as in Ref. [132], the approach 
described in this chapter can operate in-line and would be suitable for fast-tracking of the SoP in nodes of optical networks.

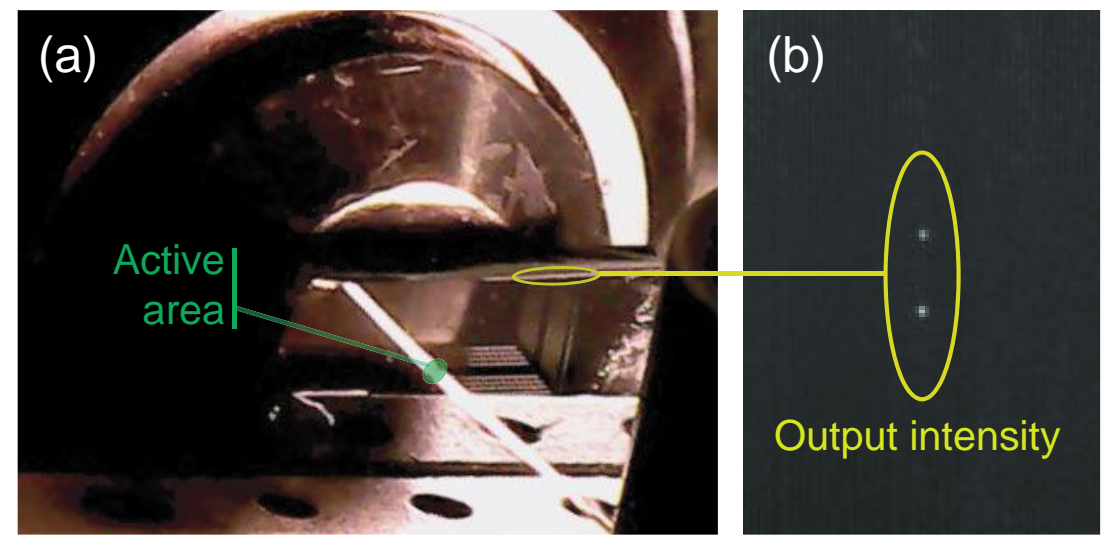

Figure 5.13 (a) Image of the sample mount in the experimental setup, where the optical fiber is aligned to the active area of the chip. The fabricated system was replicated 3 times and all output ports were redirected vertically. (b) Image of the output port spots captured by the IR camera, coupled to the microscope.

To conform the polarimetric matrix $\mathbf{W}$ the response of the polarimeter for the six "calibration polarizations" (i.e. vertical, horizontal, $45^{\circ}$ and $135^{\circ}$ linear polarizations and right, left-handed circular polarizations) is obtained. The input SoP is configured using the fiber polarization controller while monitoring the polarization with the horizontal path of the experimental setup, and the four output powers of the polarimeter are measured as explained above. Then the $\mathbf{W}$ matrix is formed from these measurements by applying Equation (5.20). The experimental result of the $\mathbf{W}$ matrix, obtained by measuring the spot intensities in camera counts of a fabricated T-block shaped polarimeter for a wavelength of $1558 \mathrm{~nm}$ is:

$$
\boldsymbol{W}=\left(\begin{array}{cccc}
54.69 & -46.24 & -42.55 & 24.34 \\
127.12 & -78.88 & 71.21 & -75.46 \\
188.10 & 3.78 & 177.99 & 66.22 \\
105.32 & 61.08 & -168.92 & -89.92
\end{array}\right)
$$

Once the polarimetric matrix was known, the active area of the nanopolarimeter was illuminated with a set of different input polarizations, where the angle, ellipticity and handedness were varied. All spot intensities for each input polarization were captured subtracting the background noise collected by the IR camera in the 
same region without spot. The experimental results of the four output spot intensities for a set of linear polarization measurements are depicted in Figure 5.14.

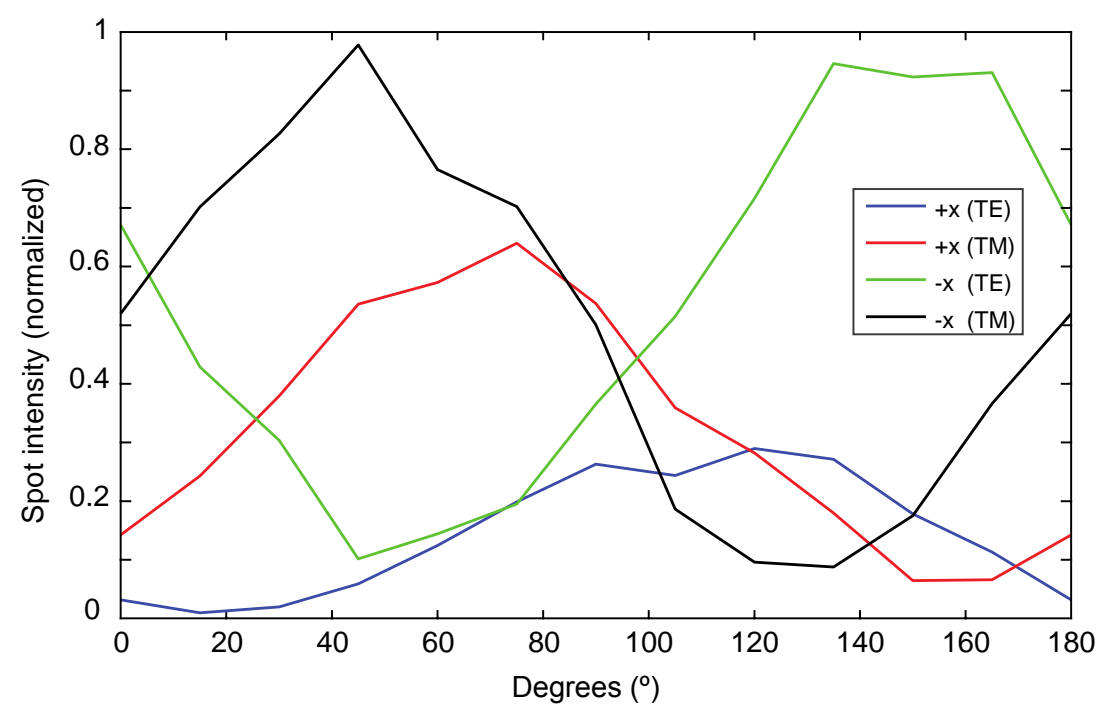

Figure 5.14 Experimentally measured output spot intensities for a set of different input linear polarizations, normalized respect to the maximum spot intensity. Note that the response of the system has a sine-like variation.

Once the output power was collected, the SoP was recovered by applying Equation (5.21). Figure 5.15 shows the performance of two measured polarimeters (the rectangular shaped polarimeter and the Tblock shaped polarimeter) by comparing the polarization ellipses of the recovered SoP (dashed line) with the input SoP measured manually (solid line) with the experimental horizontal path for each polarization. The handedness is differentiated using blue color for right-handed polarization and red color for left-handed polarization.

Moreover, these devices were tested at other wavelengths so as to ensure its broad bandwidth even though the performance is not optimal. To change the operational wavelength, the polarimeter is re-calibrated, i.e. form the polarimetric matrix $\mathbf{W}$. 

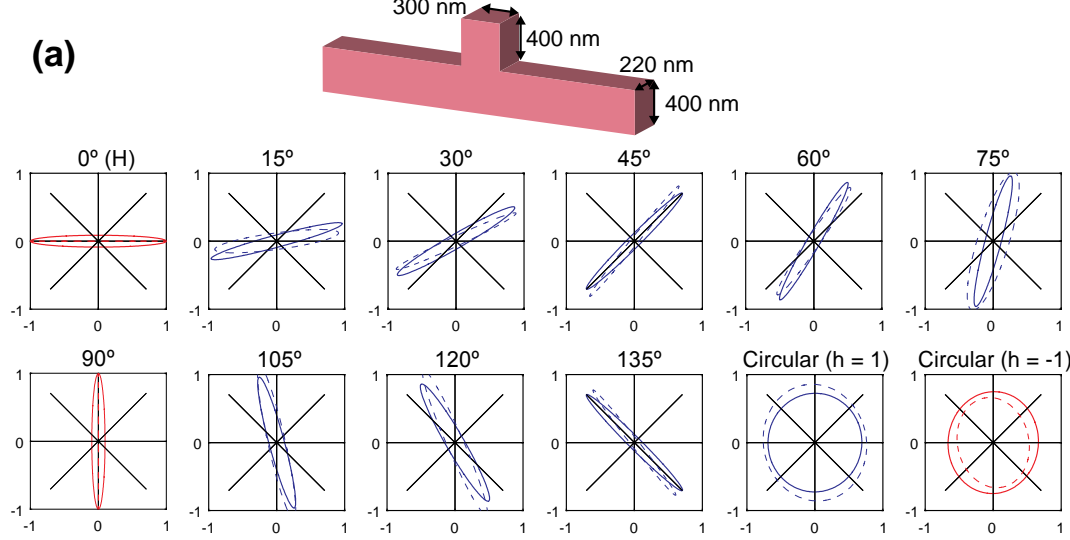

(b)
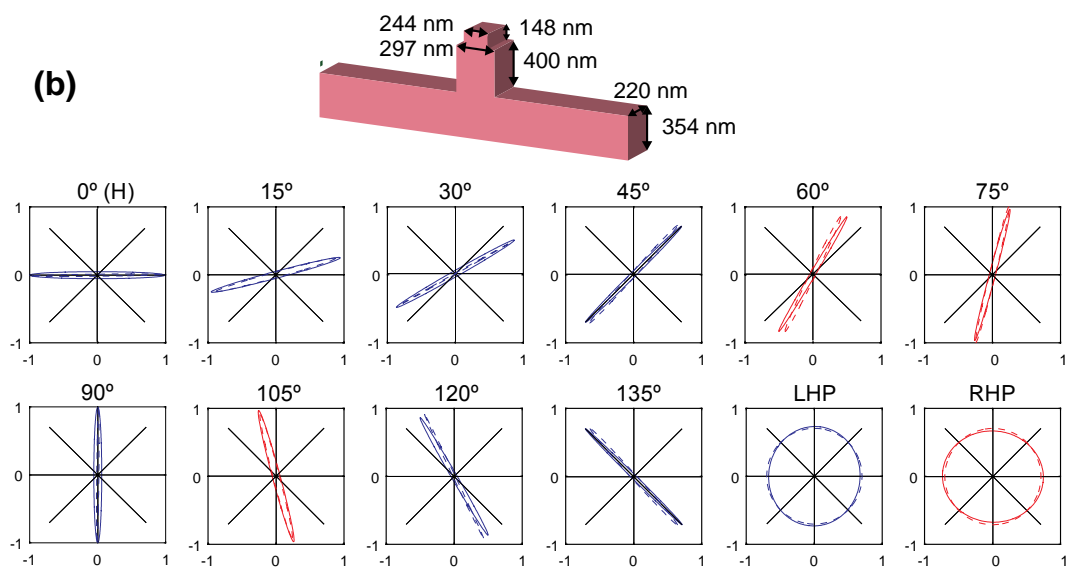

Figure 5.15 Polarization ellipses of the input (solid line) and recovered (dashed line) SoP for two different designs. (a) Response of the initial rectangular polarimeter at $\lambda=1,550 \mathrm{~nm}$; (b) Response of the optimized T-block shaped polarimeter response at $\lambda=1,558 \mathrm{~nm}$. Blue lines represent right-handed polarization and red lines represent right-handed polarization.

The results for three different wavelengths are shown in Figure 5.16, where the Stokes parameters of the input SoP (blue) and the recovered SoP by the polarimeter (red) are depicted. The selected wavelengths for testing the device were $1,550 \mathrm{~nm}, 1,520 \mathrm{~nm}$ and $1,300 \mathrm{~nm}$ for the rectangular scatterer polarimeter and $1,550 \mathrm{~nm}, 1,567 \mathrm{~nm}$ and $1,619 \mathrm{~nm}$ for the T-block shaped polarimeter, in which it is observed that the experimental results match with the expected behavior of the device. These results confirm that this device could be used for spectropolarimetry. 
(a)
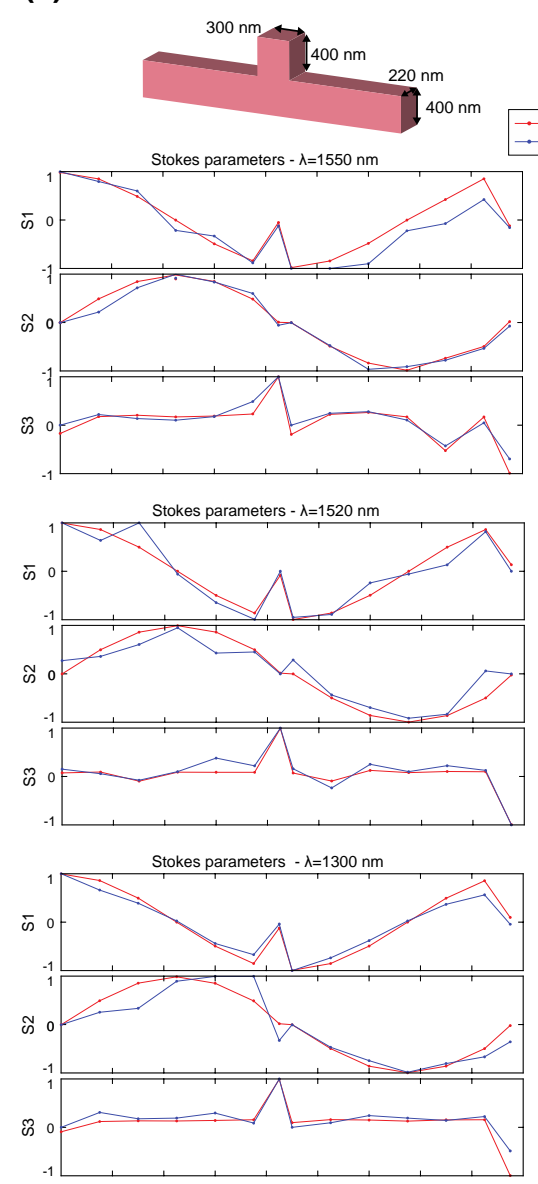

(b)

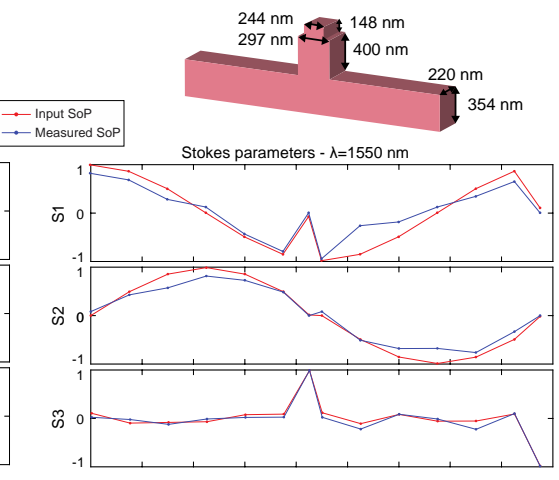

Stokes parameters $-\lambda=1567 \mathrm{~nm}$

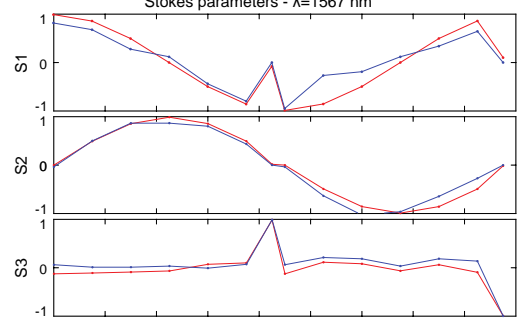

Stokes parameters $-\lambda=1619 \mathrm{~nm}$

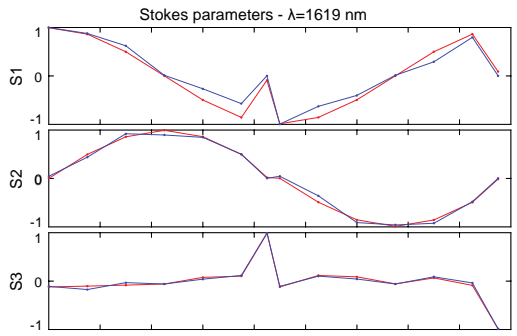

Figure 5.16 Experimental results of the input and recovered SoP represented using the Stokes parameters $S_{1}, S_{2}$ and $S_{3}$ for different wavelengths and designs. In (a) is depicted the response of the initial rectangular polarimeter for wavelengths $1,550 \mathrm{~nm}, 1,520$ $\mathrm{nm}$ and 1,300 nm, while in (b) is depicted the optimized T-block shaped polarimeter response for wavelengths $1,550 \mathrm{~nm}, 1,567 \mathrm{~nm}$ and $1,619 \mathrm{~nm}$. A set of input polarization is depicted in red, where the angle, ellipticity and handedness is modified. The recovered polarization by the Stokes nanopolarimeter is depicted in blue.

The retrieved polarizations of the optimal T-block shaped design at the center wavelength of $1558 \mathrm{~nm}$ are shown in Figure 5.17(a) in terms of the Stokes parameters. The agreement between the generated polarization (measured externally) and the polarization retrieved after 
measurement of the output power for each mode is remarkable, even for elliptical polarizations. Figure 5.17(b) shows the matrix conditioning number $\kappa$ as a function of the wavelength obtained from simulations for both the initial and the optimized nanopolarimeters. It is seen that the optimal polarimeter reaches the minimum attainable condition number $\kappa=\sqrt{3}$ at $\lambda=1,570 \mathrm{~nm}$ and performs quasi-optimally on a broad bandwidth $(\sim 100 \mathrm{~nm})$. The value of $\kappa$ is also retrieved from experiments for the optimal structure (see Figure 5.17(b)).

(a)
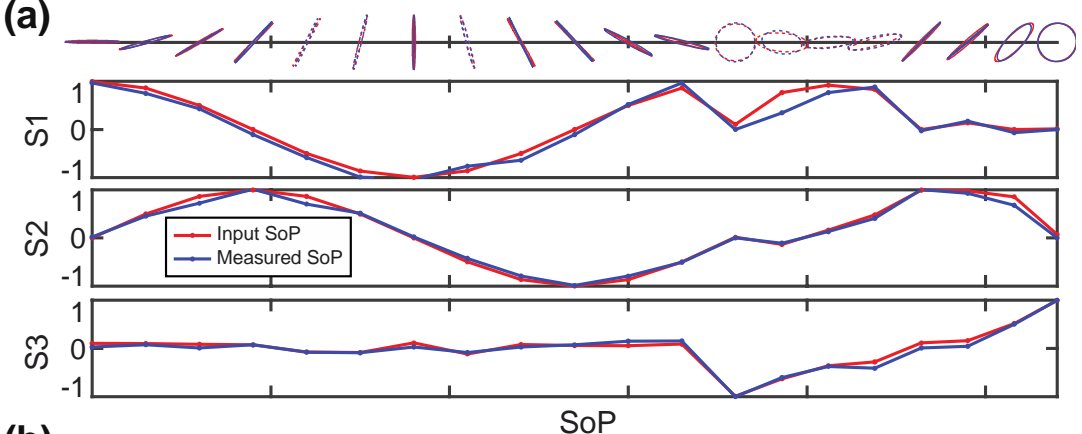

(b)

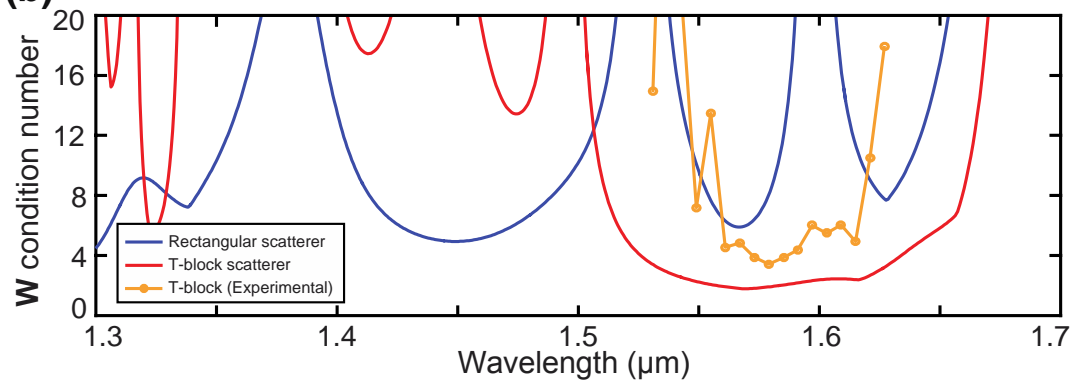

Figure 5.17 (a) Retrieved polarization for a set of experiments performed at $\lambda=1558 \mathrm{~nm}$ : input (red) and retrieved (blue) SoP. (b) Condition number $\kappa$ of the matrix $\mathbf{W}$ as a function of the operation wavelength for the initial rectangular scatterer (simulation) and the optimal scatterer (simulation and experiment).

Although the obtained values (minimum $~ 4$ ) are not so small as for the numerical simulations (which can be mainly ascribed to the fabricationinduced rounding of the scatterer, as seen in the SEM image, and to unequal propagation losses of the output waveguides), the general spectral shape shows a good qualitative correspondence with the calculated one. Notice that it is possible to retrieve the SoP at other wavelengths, even though in this case the nanopolarimeter did not 
perform optimally (see Figure 5.16). However, in general a better accuracy is observed for smaller values of $\kappa$.

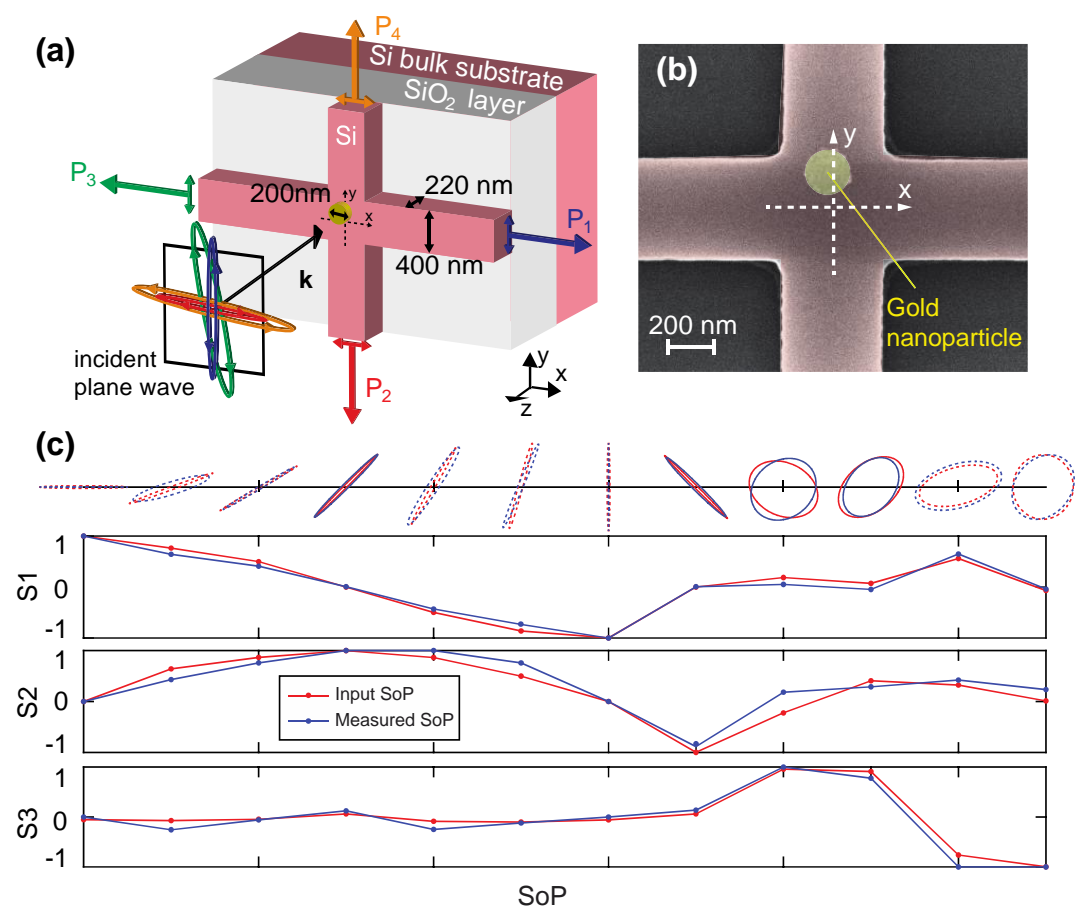

Figure 5.18 a, Scheme of the nanopolarimeter consisting of a gold microdisk asymmetrically placed on top of a silicon waveguide crossing. The polarizations maximizing the outputs are also depicted (in this case, the nanopolarimeter does not perform optimally). b, SEM image of a fabricated device (the gold microdisk has a diameter of $200 \mathrm{~nm}$ and a thickness of $30 \mathrm{~nm}$ ). c, Experimental SoP retrieval at $\lambda=1,550 \mathrm{~nm}$ : input (red) and retrieved (blue) SoP.

In the previous configuration, separation of the TE-like and TM-like guided modes is required to determine the Stokes parameters, which could be done on-chip via modal splitting architectures [146] or by employing polarization filters after light has escaped from the chip (as in the experiments). In order to avoid conversion or filtering processes, other architectures following the general scheme of Figure 5.1 could be implemented. For instance, a metallic scatterer asymmetrically deposited on top of a waveguide crossing (see Figure 5.18(a)) could be employed. In this case, by measuring the total optical power (TM + TE modes) at each of the four output ports (see the SEM image of a 
fabricated sample in Figure 5.18(b) it is possible to retrieve the Stokes parameters of the incoming signal (Figure 5.18(c)). Though the performance of the nanopolarimeter shown in Figure 5.18 is not optimal, it could be optimized by using the same approach described above.

With all of this, SOI provides a general method to fully determine the polarization of a light beam locally, non-destructively, optimally and in a single shot. This approach shows a number of advantages such as optimal operation over a broad bandwidth, on-chip implementation in a single lithography step, and suitability for high-speed polarization measurement in an in-line configuration. 


\section{Conclusions and future work}

In this Thesis, it has been shown how bringing concepts unveiled in the fields of plasmonics and nanoantennas into silicon photonics technology can result in new nanophotonic devices for manipulating light at the nanoscale. Bridging plasmonics and silicon photonics can bring benefits to both of them: Plasmonic nanostructures and nanoantennas can be benefitted by the possibility of connecting multiple elements via lossless integrated waveguides, whilst silicon photonics could find a way towards further miniaturization beyond the diffraction limit of processing elements. In some cases, such as in nanoantennas, silicon can be used instead of the metal in order to reduce undesired absorption losses, though larger elements are obtained. For this, throughout the research work of this Thesis, several objectives have been achieved such as:

- The transverse spin as well as the orbit-coupling features in silicon strip and slot waveguides have been numerically analyzed, providing cross-sectional maps that lock the position and polarization of a point-like source with the propagation direction of the excited guided mode. It has been shown that the transverse spin can be significant not only in the evanescent region but also inside the waveguide core. Unlike more symmetric waveguides such as metal plates or cylindrical fibers, silicon waveguides exhibit local points exhibiting circular polarization in transverse planes both in the evanescent and guided regions. Importantly, the spin-momentum locking producing the SCUE of guided waves is different for each guided mode. Therefore, the unidirectional excitation for a certain excitation dipole only take place for the mode satisfying the local polarization conditions imposed by the electric field components of the mode. This should be carefully taken into account in real applications, for instance, by engineering the waveguide to be single mode at the working wavelength or by externally filtering the undesired modes. These results can be relevant for studying spin-orbit coupling effects (such as the SCUE of guided modes) in a photonic integrated platform, including not only silicon but also active III-V materials, with applications in multiple disciplines ranging from quantum processing to optical processing. In addition, this envisage that the existence of relevant transverse spin must be properly considered when coupling 
plasmonic metallic scatterers or nanoantennas to silicon waveguides [56,101,102].

- It has been proved that a subwavelength metallic nanostructure can be strongly excited by a guided mode by placing it at the gap of a discontinuous silicon waveguide. The observed resonance displays an asymmetric Fano behavior as a result of the interference between the guided mode and field radiated by the metallic nanoantenna. Remarkably, the nanostructure response is characterized by a crossing between the transmission and reflection spectra in the wavelength region close to the LSPR. This approach, which can be realized using standard semiconductor nanofabrication tools, could lead to fully exploit the extreme properties of subwavelength metallic nanostructures in an on-chip configuration, and the high contrast $(>10 \mathrm{~dB}$ in measurements, > $50 \mathrm{~dB}$ in numerical simulations) observed in transmission could be extremely helpful in applications including biosensing or switching.

- A coherent approach enabling full control of the nanoantenna properties by placing it in the waveguide gap has been presented. Remarkably, a single nanoantenna placed on top of the waveguide, which is easier from the fabrication point of view, does not provide the sufficient level of absorption or scattering $(<20 \%$ in $\mathrm{SiN}$ waveguides[56,102], $<10 \%$ in Silicon waveguides[147]) as to produce a large extinction in single-side illumination [56] and then CPE in guided light. Indeed, arrays of plasmonic elements are usually integrated in order to increase the total interaction and produce observable extinction at the output waveguide.[46,55,100,101,102,62]. This is the reason why achieving large contrast ratios at the output and ultimately CPE requires placing an array of nanoantennas on the waveguide top [148]. Modulation of the scattering of a nanoantenna placed on top of a waveguide was also recently shown experimentally, being the interaction enhanced by using dielectric resonant cavities [149]. Other works have addressed analytically and numerically the coherent suppression or enhancement of absorption in photonic integrated circuits by using large-size structures [150]. However, the use of embedded plasmonic nanostructures fully illuminated provides the right platform for completely controlling the nanoantenna scattering as well as achieving large intensity modulation of the guided light in a subwavelength-size foot-print. 
- It has been shown that coherent interference using orthogonal paths also provides a versatile tool for polarization synthesis. Since the nanoantenna used in this Thesis displays a large scattering crosssection, it becomes feasible to achieve a large modulation of the scattering. However, it is not possible to reach the CPA regime which would enable large intensity modulation of on-chip guided signals. However, by using a nanoantenna tailored to support a non-radiative anapole mode [151] or using scattering cancellation techniques [152] the CPA regime in a subwavelength region and over a large bandwidth could be attained. Then, all coherent processing devices proposed in Ref. [112] could be implemented on a silicon chip. The described system is also suitable for dense integration of multiple nanoantennas on a chip, which could lead to massive multiplexed coherent all-optical signal processing.

- An optimal Stokes nanopolarimeter has been designed, which is fully fabricated on a silicon chip without metals, makes use of the SOI taking place in a subwavelength scatterer meaning that $\mathrm{SoP}$ is detected locally. Notice that polarimeters relying upon plasmonic nanoresonators $[129,130]$ and metasurfaces $[131,132,133,134,135]$ have been recently demonstrated. Despite their remarkable performance, they use metallic nanostructures with complex shapes, which besides introducing undesired losses, are not typically compatible with standard semiconductor fabrication technologies. More importantly, they do not allow local measurement of the SoP: whilst plasmonic resonators measure distinct polarization components in different places, metasurfaces are based on an extended, collective response of a set of scatterers. However, the presented proposal overcomes all these obstacles. Moreover, the nanopolarimeter can be designed to operate optimally, which would reduce the effect of noise and random errors arising during the retrieval process. Notice that recently proposed metasurface-based polarimeters cannot operate optimally since the states they separate always describe a plane in the Poincaré sphere [132]. Since the underlying silicon substrate is transparent at the operating wavelength, the nanopolarimeter can be inserted directly on the optical path of a light beam, with only a negligible fraction of the incident power being sampled and used for measurement, while the rest of the beam propagates through the silicon chip. Such in-line operation with low insertion losses allows measurement of the SoP of a light beam in real time with little disturbance to the beam (non-destructive). This contrasts with 
recently proposed methods of local measurement of vector electric field that rely on collecting scattered light over a wide range of angles [153]. Another remarkable benefit of the SOI nanopolarimeter is that its outputs are integrated waveguides, therefore potentially allowing on-chip measurement and SoP retrieval with no external optics or cameras. The use of nanophotonic waveguides as paths for the SoP information provides other advantages: one-dimensional arrays for simultaneous SoP scanning along a line could be easily constructed, and the SoP mapping could be further processed (for instance, measuring the spectrum via on-chip spectrometers [154] could lead to on-chip nano-spectropolarimetry).

- The SOI Stokes nanopolarimetry method is universal: it can be applied to any frequency regime, being always subwavelength in size, and implemented in any technological platform. Together with the integrated approach for generation of arbitrary polarization states [29], the nanopolarimeter completes a set of nanophotonic elements for full local polarization management at the nanoscale, unveiling the practical potential of the QSHE of light [65].

So, bringing plasmonics and nanoantenna concepts into silicon photonics can be disruptive in many applications, including sensing, spectroscopy, polarization managing and high-speed data processing. 


\section{Methods}

\section{Numerical simulations}

Numerical simulations in this Thesis have been performed using the commercial 3-D full-wave solver CST Microwave Studio, which implements finite integration technique. Unlike in most numerical methods that solve Maxwell's equations in a differential form, the FIT method consists in the resolution of Maxwell's equations in an integral form. To solve these equations numerically, it is necessary to discretize the calculation domain of the considered problem. Therefore, an adequate mesh is necessary to divide the problem into cells. Generally, in the structures described in this Thesis, hexahedral mesh with 10 cells per wavelength has been used, except in metallic inclusions, where the mesh has been refined up to approximately $15 \mathrm{~nm}$ (reaching around one hundred cells in total). Open boundary conditions (perfectly matched layers) have been chosen for all external faces. Metallic elements have been made of gold, whose optical constants were obtained from ellipsometry measurements of thin films deposited using the same procedure as described in the fabrication section.

For waveguide mode parameters, calculations have been performed using a finite-element method implemented in the commercial package FemSIM $^{\mathrm{TM}}$ by RSoft. A grid size of $20 \mathrm{~nm}$ has been considered.

\section{Near field measurements}

Figure 0.1(a) shows a scheme of the set-up employed for the Scanning Near-field Optical Microscopy (SNOM) measurements. The measurements were performed with a tailored MultiView 4000 system (Nanonics Imagin Ltd) working in collection mode. A bent fiber tip (Nanonics Imaging Ltd) with a $400 \mathrm{~nm}$ aperture, $\mathrm{Cr} / \mathrm{Au}$ coated, premounted on a tuning-fork working in tapping mode at $36.94 \mathrm{kHz}$, was used to scan the lithographed sample while it was kept fixed (see Figure 0.1(b)). The sample was laterally illuminated with a lensed fiber mounted on a 3D stage. The whole system can be pre-visualized by a vertical optical microscope that enables the correct alignment and to select the accurate positioning of the SNOM probe on top of the lithographed structures (see Figure 0.1(c)). The lensed fiber was connected to a tunable laser (Keysight Agilent Laser, 81940A) at a fixed light polarization and a power of $14 \mathrm{dBm}$. 


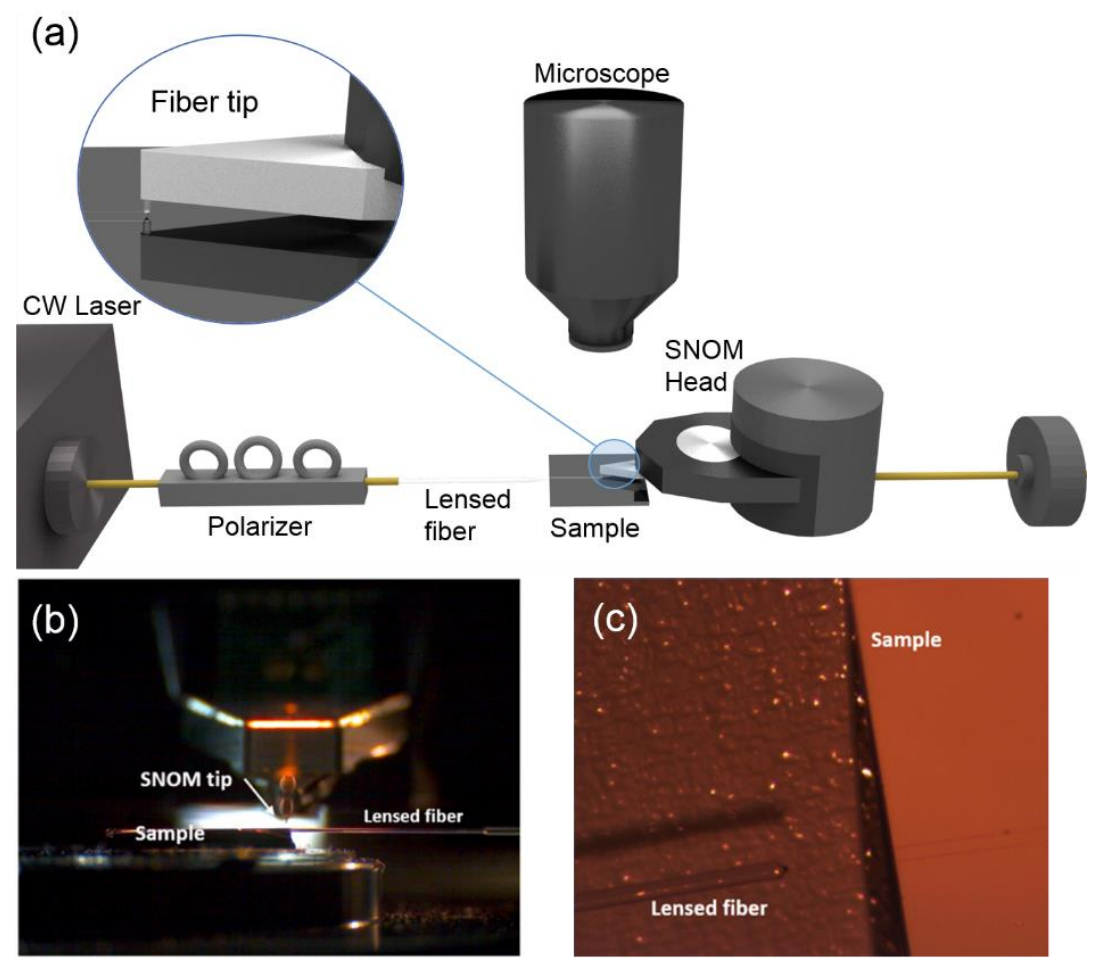

Figure 0.1 (a) Schematic view of the set-up. (b) Optical capture SNOM setup for lateral illumination. (c) Top view optical image of lateral illumination.

\section{Fabrication}

Throughout this Thesis several samples of the studied designs have been fabricated. In terms of fabrication, two groups can be considered: the metallic-silicon hybrid structures (Chapters 3, 4 and 5), and the silicon structures (Chapter 5).

Silicon structures were fabricated on standard silicon-on-insulator samples from SOITEC wafers with a top silicon layer thickness of 220 $\mathrm{nm}$ and a buried oxide layer thickness of $2 \mu \mathrm{m}$. Patterns were defined by using an electron-beam direct-writing process performed on a coated $100 \mathrm{~nm}$ hydrogen silsesquioxane (HSQ) resist film. The electron-beam exposure, performed with a Raith150 tool, was optimized to reach the required dimensions employing an acceleration voltage of $30 \mathrm{keV}$ and an aperture size of $30 \mu \mathrm{m}$. After developing the HSQ film using 
tetramethylammonium hydroxide, the resist patterns were transferred into the samples by employing an optimized Inductively Coupled Plasma-Reactive Ion Etching process with fluoride gases.

For hybrid metal-silicon samples (see Chapters 3-4), the selected wafers have been different in order to have a larger cross section of the waveguide and facilitate the embedding of the metal nanoparticles. In those cases, $250 \mathrm{~nm}$ silicon core on top of a $3 \mu \mathrm{m}$ buried oxide substrate wafers have been used. After etching, a 105 nm-thickness silicon dioxide layer was deposited on the SOI sample by using a plasma enhanced chemical vapor deposition (PECVD) system from Applied Materials. This layer is deposited to center the metallic nanostructure with the optical axis of the waveguide, ensuring maximum interaction with the field within the gap. A second e-beam exposure prior to a metal evaporation and lift-off processes were carried out in order to define the $40 \mathrm{~nm}$ thickness gold nanoparticle inside the waveguide gaps. A $2 \mathrm{~nm}$ titanium layer were also evaporated to improve gold adhesion. Notice that this last step would allow for inserting metallic nanostructures with more complex shapes, such as split-ring resonators or bowtie nanoantennas. Finally, a micron-thickness silicon dioxide uppercladding was deposited on the SOI sample by using again PECVD in order to ensure homogeneity in the surroundings of the metallic nanostructure.

The fabrication process described here has been carried out entirely at the Nanophotonics Technology Center of Valencia.

\section{Fundings}

Personal financial support is gratefully acknowledged to the Spanish Ministry of Economy and Competitiveness (MINECO) under grant BES-2015073146 and TEC2014-51902-C2-1-R. 


\section{References}

1. S.A. Maier, "Plasmonics: Fundamentals and Applications," Berlin: Springer (2010).

2. J. A. Schuller, E. S. Barnard, W. Cai, Y. C. Jun, J. S. White, and M. L. Brongersma, "Plasmonics for extreme light concentration and manipulation," Nat. Materials 9, 193-204 (2010).

3. P. Zijlstra, P. M. R. Paulo and M. Orrit. "Optical detection of single non-absorbing molecules using the surface plasmon resonance of a gold nanorod," Nat. Nanotechnol. 7, 379-82 (2012).

4. A. G. Curto, G. Volpe, T. H. Taminiau, M. P. Kreuzer, R. Quidant and N. F. van Hulst, "Unidirectional emission of a quantum dot coupled to a nanoantenna," Science 329, 930-3 (2010).

5. D. R. Smith, J. B. Pendry and M. C. K. Wiltshire, "Metamaterials and negative refractive index," Science 305, 788-92 (2004).

6. D. Schurig, J. J. Mock, B. J. Justice, S. A. Cummer, J. B. Pendry, A. F. Starr and D. R. Smith, "Metamaterial electromagnetic cloak at microwave frequencies," Science 314, 977-80 (2006).

7. M. Lorente-Crespo, L. Wang, R. Ortuño, C. García-Meca, Y. Ekinci and A. Martínez, "Magnetic hot spots in closely spaced thick gold nanorings," Nano Lett. 13, 2654-61 (2013).

8. X. Ni, N. K. Emani, A. V. Kildishev, A. Boltasseva and V. M. Shalaev, "Broadband light bending with plasmonic nanoantennas," Science 335, 427 (2012).

9. N. Yu, P. Genevet, M. A. Kats, F. Aieta, J. P. Tetienne, F. Capasso and Z. Gaburro, "Light propagation with phase discontinuities: generalized laws of reflection and refraction," Science 334, 333-7 (2011).

10. M. Khorasaninejad, W. T. Chen, R. C. Devlin, J. Oh, A. Y. Zhu and F. Capasso, "Metalenses at visible wavelengths: diffractionlimited focusing and subwavelength resolution imaging," Science 352, 1190-4 (2016).

11. H. Aouani, M. Rahmani, M. Navarro-Cía, and S. A. Maier, "Thirdharmonic-upconversion enhancement from a single semiconductor nanoparticle coupled to a plasmonic antenna," Nat. Nanotechnology 9, 290-294 (2014). 
12. P. Fan, Z. Yu, S. Fan and M. L. Brongersma, "Optical Fano resonance of an individual semiconductor nanostructure," Nat. Materials 13, 471-475 (2014).

13. T. Coenen, F. Bernal-Arango, A. Femius-Koenderink and A. Polman, "Directional emission from a single plasmonic scatterer," Nat. Commun. 5, 3250 (2014).

14. D. O’Connor, P. Ginzburg, F. J. Rodríguez-Fortuño, G. A. Wurtz and A. V. Zayats, "Spin-orbit coupling in surface plasmon scattering by nanostructures," Nature Comm. 5, 5327 (2014).

15. J. K. Gansel, M. Thiel, M. S. Rill, M. Decker, K. Bade, V. Saile, G. von Freymann, S. Linden and M. Wegener, "Gold helix photonic metamaterial as broadband circular polarizer," Science 325, 1513-5 (2009).

16. Editorial, "Commercializing plasmonics," Nat. Photon. 9, 477-477 (2015).

17. L. Novotny and N. F. van Hulst, "Antennas for light," Nat. Photonics 5, 83-90 (2011).

18. P. Mühlschlegel, H. J. Eisler, O. J. F. Martin, B. Hecht and D. W. Pohl, "Resonant optical antennas" Science 308, 1607-9 (2005).

19. M. Schnell, A. García-Etxarri, A. J. Huber, K. Crozier, J. Aizpurua and R. Hillenbrand, "Controlling the near- field oscillations of loaded plasmonic nanoantennas," Nat. Photon. 3, 287-91 (2009).

20. L. Tang, S. E. Kocabas, S. Latif, A. K. Okyay, D. S. Ly-Gagnon, K. C. Saraswat and D. A. B. Miller, "Nanometre-scale germanium photodetector enhanced by a near-infrared dipole antenna," Nat. Photon. 2, 226-9 (2008).

21. N. Liu, M. L. Tang, M. Hentschel, H. Giessen and A. P. Alivisatos, "Nanoantenna-enhanced gas sensing in a single tailored nanofocus," Nat. Mater. 10, 631-6 (2011).

22. T. Schumacher, K. Kratzer, D. Molnar, M. Hentschel, H. Giessen and M. Lippitz, "Nanoantenna-enhanced ultrafast nonlinear spectroscopy of a single gold nanoparticle," Nat. Commun. 2, 333 (2011).

23. C. A. Balanis, "Antenna Theory: Analysis and Design," New York: Wiley (1997).

24. D. M. Pozar, "Microwave Engineering," New York: Wiley (2004). 
25. C. Koos, P. Vorreau, T. Vallaitis, P. Dumon, W. Bogaerts, R. Baets, B. Esembeson, I. Biaggio, T. Michinobu, F. Diederich, W. Freude, and J. Leuthold, "All-optical high-speed signal processing with silicon-organic hybrid slot waveguides", Nat. Photonics 3, no. 4, pp. 216-219, Apr. (2009).

26. A. Martínez, J. Blasco, P. Sanchis, J. V. Galán, J. García-Rupérez, E. Jordana, P. Gautier, Y. Lebour, S. Hernández, R. Guider, N. Daldosso, B. Garrido, J.-M. Fedeli, L. Pavesi, and J. Martí, "Ultrafast all-optical switching in a silicon nanocrystal-based silicon slot waveguide at telecom wavelengths," Nano Lett. 10, 1506-11 (2010).

27. M. A. Foster, A. C. Turner, J. E. Sharping, B. S. Schmidt, M. Lipson and A. L Gaeta, "Broad-band optical parametric gain on a silicon photonic chip," Nature 441, 960-3 (2006).

28. J. Sun, E. Timurdogan, A. Yaacobi, E. S. "Hosseini and M. R. Watts, Large-scale nanophotonic phased array," Nature 493, 1959 (2013).

29. F. J. Rodríguez-Fortuño, D. Puerto, A. Griol, L. Bellieres, J. Martí, A. Martínez, "Universal method for the synthesis of arbitrary polarization states radiated by a nanoantennas," Laser Photonics Rev. 8(3), L27-L31 (2014).

30. A. Alù and N. Engheta, "Input impedance, nanocircuit loading, and radiation tuning of optical nanoantennas," Phys. Rev. Lett. 101, 43901 (2008).

31. J. S. Huang, T. Feichtner, P. Biagioni and B. Hecht, "Impedance matching and emission properties of nanoantennas in an optical nanocircuit," Nano Lett. 9, 1897-902 (2009).

32. A. Alù and N. Engheta, "Wireless at the nanoscale: optical interconnects using matched nanoantennas," Phys. Rev. Lett. 104, $1-4$ (2010).

33. D. Dregely, R. Taubert, J. Dorfmüller, R. Vogelgesang, K. Kern and H. Giessen, "3D optical Yagi-Uda nanoantenna array," Nat. Commun. 2, 267 (2011).

34. Editorial, "Focus on 'silicon photonics'," Nat. Photon. 4, 491 (2010).

35. M. Hochberg and T. Baehr-Jones, "Towards fabless silicon photonics," Nat. Photon. 4, 492-4 (2010). 
36. A. Rickman, "The commercialization of silicon photonics," Nat. Photon. 8, 579-82 (2014).

37. T. Baehr-Jones, T. Pinguet, P. Lo Guo-Qiang, S. Danziger, D. Prather and M. Hochberg, "Myths and rumours of silicon photonics," Nat. Photon. 6, 206-8 (2012).

38. T. Claes, W. Bogaerts and P. Bienstman, "Vernier-cascade labelfree biosensor with integrated arrayed waveguide grating for wavelength interrogation with low-cost broadband source," Opt. Lett. 36, 3320 (2011).

39. M. C. Estevez, M. Alvarez and L. M. Lechuga, "Integrated optical devices for lab-on-a-chip biosensing applications," Laser Photon. Rev. 6, 463-87 (2012).

40. J. G. Castelló, V. Toccafondo, P. Pérez-Millán, N. S. Losilla, J. L. Cruz, M. V. Andrés and J. García-Rupérez, "Real-time and lowcost sensing technique based on photonic bandgap structures," Opt. Lett. 36, 2707 (2011).

41. C. Sun et al, "Single-chip microprocessor that communicates directly using light," Nature 528, 534-8 (2015).

42. L. Vivien, "Computer technology: silicon chips lighten up," Nature 528, 483-4 (2015).

43. Genalyte, http://genalyte.com/.

44. Intel, http://intel.com/content/www/us/en/research/intel-labssilicon-photonics-research.html.

45. M. Gnan, S. Thoms, D. S. Macintyre, R. M. De La Rue and M. Sorel, "Fabrication of low-loss photonic wires in silicon-oninsulator using hydrogen silsesquioxane electron-beam resist," Electron. Lett. 44, 115 (2008).

46. F. B. Arango, A. Kwadrin, and A. F. Koenderink, "Plasmonic antennas hybridized with dielectric waveguides," ACS Nano 6 , 10156 (2012).

47. C. T. DeRose, R. D. Kekatpure, D. C. Trotter, A. Starbuck, J. R. Wendt, A. Yaacobi, M. R. Watts, U. Chettiar, N. Engheta and P. S. Davids, "Electronically controlled optical beam-steering by an active phased array of metallic nanoantennas," Opt. Express 21, 5198 (2013). 
48. M. L. Brongersma and V. M. Shalaev, "The case for plasmonics," Science 498, 2009-10 (2010).

49. N. Engheta, "Circuits with light at nanoscales: optical nanocircuits inspired by metamaterials," Science 317, 1698-702 (2007).

50. J. Leuthold, C. Koos and W. Freude, "Nonlinear silicon photonics," Nat. Photon. 4, 535-44 (2010).

51. K. F. MacDonald, Z. L. Sámson, M. I. "Stockman and N. I. Zheludev, Ultrafast active plasmonics," Nat. Photon. 3, 55-8 (2009).

52. M. Kauranen, A.V. Zayats, "Nonlinear plasmonics," Nat. Photonics 6(11), 737-748 (2010).

53. G. A. Wurtz, R. Pollard, W. Hendren, G. P. Wiederrecht, D. J. Gosztola, V. A. Podolskiy and A. V. Zayats, "Designed ultrafast optical nonlinearity in a plasmonic nanorod metamaterial enhanced by nonlocality," Nat. Nanotechnol. 6, 107-11 (2011).

54. T. P. H. Sidiropoulos, M. P. Nielsen, T. R. Roschuk, A. V. Zayats, S. A. Maier and R. F. Oulton, "Compact optical antenna coupler for silicon photonics characterized by third- harmonic generation," ACS Photon. 1, 912-6 (2014).

55. F. Peyskens, A. Dhakal, P. Van Dorpe, N. Le Thomas, and R. Baets, "Surface enhanced Raman spectroscopy using a single mode nanophotonic-plasmonic platform," ACS Photon. 3, 102 (2016).

56. M. Chamanzar, Z. Xia, S. Yegnanarayanan, and A. Adibi, "Hybrid integrated plasmonic-photonic waveguides for on-chip localized surface plasmon resonance (LSPR) sensing and spectroscopy," Opt. Express 21(26), 32086-32098 (2013).

57. C. Haffner et al, "All-plasmonic Mach-Zehnder modulator enabling optical high-speed communication at the microscale," Nat. Photon. 9, 525-8 (2015).

58. A. D. Neira, G. A. Wurtz, P. Ginzburg and A. V. Zayats, "Ultrafast all-optical modulation with hyperbolic metamaterial integrated in Si photonic circuitry," Opt. Express 22, 10987 (2014).

59. K. Y. Bliokh, A. Y. Bekshaev, and F. Nori, "Extraordinary momentum and spin in evanescent waves," Nat. Commun 5, 3300 (2014). 
60. A. Espinosa-Soria and A. Martínez, "Transverse spin and spin-orbit coupling in silicon waveguides," IEEE Photon. Technol. Lett. 28(14), 1561-1564 (2016).

61. R. J. Coles, D. M. Price, J. E. Dixon, B. Royall, E. Clarke, A. M. Fox, P. Kok, M. S. Skolnick, and M. N. Makhonin, "Chirality of nanophotonic waveguide with embedded quantum emitter for unidirectional spin transfer," Nat. Commun. 7, 11183 (2016).

62. M. Castro-Lopez, N. de Sousa, A. Garcia-Martin, F. Y. Gardes, and R. Sapienza, "Scattering of a plasmonic nanoantenna embedded in a silicon waveguide," Opt. Express 23(22), 28108-18 (2015).

63. I. M. Hancu, A. G. Curto, M. Castro-López, M. Kuttge, and N. F. van Hulst, "Multipolar interference for directed light emission," Nano Lett. 14, 166-171 (2014).

64. D. Vercruysse, Y. Sonnefraud, N. Verellen, F. B. Fuchs, G. Di Martino, L. Lagae, V. V. Moshchalkov, S. A. Maier, and P. Van Dorpe, "Unidirectional side scattering of light by a single-element nanoantenna," Nano Lett. 13, 3843-3849 (2013).

65. K. Y. Bliokh, D. Smirnova, and F. Nori, "Quantum spin Hall effect of light”, Science 348(6242), 1448-1451 (2015).

66. F. J. Rodríguez-Fortuño, G. Marino, P. Ginzburg, D. O’Connor, A. Martínez, G. A. Wurtz, and A. V. Zayats, "Near-Field Interference for the Unidirectional Excitation of Electromagnetic Guided Modes," Science 340(6130), 328-330 (2013).

67. A. Aiello, P. Banzer, M. Neugebauer and G. Leuchs, "From transverse angular momentum to photonic wheels," Nat. Photon. 9, 789-95 (2015).

68. M. Neugebauer, T. Bauer, P. Banzer, and G. Leuchs, "Polarization Tailored Light Driven Directional Optical Nanobeacon," Nano Lett. 14(5), 2546-2551 (2014).

69. J. Petersen, J. Volz, and A. Rauschenbeutel, "Chiral nanophotonic waveguide interface based on spin-orbit interaction of light", Science 346(620), 67-71 (2014).

70. F. J. Rodríguez-Fortuño, D. Puerto, A. Griol, L. Bellieres, J. Martí, and A. Martínez, "Sorting linearly polarized photons with a single scatterer" Opt. Lett. 39(6), 1394-1397 (2014).

71. A. Espinosa-Soria, S. Mas, A. Griol, F. J. Rodríguez-Fortuño and A. Martínez, "Full Measurement of the Stokes Parameters of a 
Light Beam using On-Chip Silicon Nanoantennas," in 9th International Congress on Advanced Electromagnetic Materials in Microwaves and Optics. Oxford (2015).

72. L. Marrucci, "Quantum optics: spin gives direction," Nat. Phys. 11, 9-10 (2014).

73. C. Stanciu, F. Hansteen, A. Kimel, A. Kirilyuk, A. Tsukamoto, A. Itoh and T. Rasing, "All-optical magnetic recording with circularly polarized light,” Phys. Rev.Lett. 9947601 (2007).

74. K. Y. Bliokh, A. Y. Bekshaev, and F. Nori, "Transverse spin of a surface polariton," Phys. Rev. A 85(6), 061801 (2012).

75. M. R. Dennis and J. B. Götte, "Beam shifts for pairs of plane waves," J. Opt. 15, 014015 (2013).

76. P. V. Kapitanova, P. Ginzburg, F. J. Rodriguez-Fortuno, D. S. Filonov, P. M. Voroshilov, P. A. Belov, A. N. Poddubny, Y. S. Kivshar, G. A. Wurtz, and A. V. Zayats, "Photonic spin Hall effect in hyperbolic metamaterials for polarization-controlled routing of subwavelength modes," Nat. Commun. 3326 (2014).

77. F. J. Rodríguez-Fortuño, I. Barber-Sanz, D. Puerto, A. Griol, and A. Martínez, "Resolving light handedness with an on-chip silicon microdisk," ACS Photonics 1(9), 762-767 (2014).

78. B. le Feber, N. Rotenberg, and L. Kuipers, "Nanophotonic control of circular dipole emission," Nature Comm. 6, 6695 (2015).

79. Y. Lefier and T. Grosjean, "Unidirectional sub-diffraction waveguiding based on optical spin-orbit coupling in subwavelength plasmonic waveguides," Opt. Lett. 40(12), 28902893 (2015).

80. T. V. Mechelen and Z. Jacob, "Universal spin-momentum locking of evanescent waves", Optica 3,118 (2016).

81. J. Carbonell, F. J. Rodríguez-Fortuño, A. Díaz-Rubio, A. Martínez, F. Cervera, and J. Sánchez-Dehesa, "Directive excitation of guided electromagnetic waves through polarization control," Phys. Rev. B 89(15), 155121 (2014).

82. M. Neugebauer, T. Bauer, A. Aiello, and P. Banzer, "Measuring the Transverse Spin Density of Light," Phys. Rev. Lett. 114(6), 063901 (2015). 
83. C. Junde, D. O'Shea, J. Volz and A. Rauschenbeutel, "Strong coupling between single atoms and nontransversal photons," Phys. Rev. Lett. 110(21), 213604 (2013).

84. D. Jukiç, T. Pohl and J. B. Götte, "Evanescent fields of laser written waveguides," in Proc. SPIE Complex light and optical forces IX, San Francisco, CA, 93790R, (2015).

85. F. Belinfante, "On the current and the density of the electric charge, the energy, the linear momentum and the angular momentum of arbitrary fields," Physica 7, 449-474 (1940).

86. V. R. Almeida, Q. Xu, C. A. Barrios, and M. Lipson, "Guiding and confining light in void nanostructure," Opt. Lett. 29(11) 12091211 (2004).

87. M. Burresi, R. J. P. Engelen, A. Opheij, D. van Oosten, D. Mori, T. Baba, and L. Kuipers, "Observation of polarization singularities at the nanoscale," Phys. Rev. Lett. 102(3), 033902 (2009).

88. C. A. Barrios, M. J. Bañuls, V. González-Pedro, K. B. Gylfason, B. Sánchez, A. Griol, A. Maquieira, H. Sohlström, M. Holgado, and R. Casquel, "Label-free optical biosensing with slot-waveguides," Opt. Lett. 33(7), 708-710 (2008).

89. A. V. Zayats and I. I. Smolyaninov, "Near-field photonics: surface plasmon polaritons and localised surface plasmons," J. Opt. A, Pure Appl. Opt. 5(4), S16-S50 (2003).

90. D. K. Gramotnev and S. I. Bozhelvonyi, "Plasmonics beyond the diffraction limit," Nat. Photonics 4(2), 83-91 (2010).

91. J. N. Anker, W. P. Hall, O. Lyandres, N. C. Shah, J. Zhao and R. P. Van Duyne, "Biosensing with plasmonic nanosensors," Nat. Materials 7, 442-453 (2008).

92. M. Février, P. Gogol, G. Barbillon, A. Aassime, R. Mégy, B. Bartenlian, J. Lourtioz and B. Dagens, "Integration of short gold nanoparticles chain on SOI waveguide toward compact integrated bio-sensors," Opt. Express 20, 17402-9 (2012).

93.M. Husnik, M. W. Klein, N. Feth, M. König, J. Niegemann, K. Busch, S. Linden, and M. Wegener, "Absolute Extinction Cross Section of Individual Magnetic Split-Ring Resonators," Nat. Photonics 2(10), 614-617 (2008).

94.P.J. Rodríguez-Cantó, M. Martínez-Marco, F. J. RodríguezFortuño, B. Tomás-Navarro, R. Ortuño, S. Peransí-Llopis, and A. 
Martínez, "Demonstration of near infrared gas sensing using gold nanodisks on functionalized silicon," Opt. Express 19(8), 76647672 (2011).

95.M. Lipson, "Guiding, modulating, and emitting light on silicon Challenges and opportunities," J. Lightwave Technol. 23, 42224238 (2005)

96.M. Hochberg and T. Baehr-Jones, "Toward fabless silicon photonics," Nat. Photonics 4, $492-494$ (2010).

97.A. Rickman, "The commercialization of silicon photonics," Nat. Photonics 8, 579-582 (2014).

98.N. Daldosso, M. Melchiorri, F. Riboli, F. Sbrana, L. Pavesi, G. Pucker, C. Kompocholis, M. Crivellari, P. Belluti, and A. Lui, "Fabrication and optical characterization of thin two-dimensional Si3N4 waveguides," Mater. Sci. Semicond. Process. 7, 453-458 (2004).

99.S. Romero-García, F. Merget, F. Zhong, H. Finkelstein, and J. Witzens, "Silicon nitride CMOS-compatible platform for integrated photonics applications at visible wavelengths," Opt. Express 21, 14036-14046 (2013)

100. I. Alepuz-Benache, C. García-Meca, F. J. Rodríguez-Fortuño, R. Ortuño, M. Lorente-Crespo, A. Griol, and A. Martínez, "Strong magnetic resonance of coupled aluminum nanodisks on top of a silicon waveguide," Proc. SPIE 8424, 84242J (2012).

101. M. Février, P. Gogol, A. Aassime, R. Mégy, C. Delacour, A. Chelnokov, A. Apuzzo, S. Blaize, J-M. Lourtioz, and B. Dagens, "Giant coupling effect between metal nanoparticle chain and optical waveguide," Nano Lett. 12, 1032-1037 (2012).

102. F. Peyskens, A. Z. Subramanian, P. Neutens, A. Dhakal, P. Van Dorpe, N. Le Thomas, and R. Baets, "Bright and dark plasmon resonances of nanoplasmonic antennas evanescently coupled with a silicon nitride waveguide," Opt. Express 23(3) 3088-3101 (2015).

103. F. Bernal Arango, R. Thijssen, B. Brenny, T. Coenen and A. Femius Koenderink, "Robustness of plasmon phased array nanoantennas to disorder," Sci. Rep. 5, 10911 (2015). 
104. M. W. Klein, C. Enkrich, M. Wegener, C. M. Soukoulis, and S. Linden, "Single-slit split-ring resonators at optical frequencies: limits of size scaling," Opt. Lett. 31, 1259-1261 (2006).

105. N. Verellen, Y. Sonnefraud, H. Sobhan, F. Hao, V. V. Moshchalkov, P. Van Dorpe, P. Nordlander and Stefan A. Maier, "Fano resonances in individual coherent plasmonic nanocavities," Nano Lett. 9, 1663-1667 (2009).

106. U. K. Chettiar, R. Fernandez-Garcia, S. A. Maier, and N. Engheta, "Enhancement of radiation from dielectric waveguides using resonant plasmonic coreshells," Opt. Express 20(14), 1610416112 (2012).

107. B. Luk'yanchuk, N. I. Zheludev, S. A. Maier, N. J. Halas, P. Nordlander, H. Giessen, and C. T. Chong, "The Fano resonance in plasmonic nanostructures and metamaterials," Nat. Mater. 9(9), 707-15 (2010).

108. S. Hamed Shams Mousavi, Ali A. Eftekhar, Amir H. Atabaki, and Ali Adibi, "Band-edge bilayer plasmonic nanostructure for surface enhanced Raman spectroscopy," ACS Photon. 2, 1546-1551 (2015).

109. N. Verellen, F. López-Tejeira, R. Paniagua-Domínguez, D. Vercruysse, D. Denkova, L. Lagae, P. Van Dorpe, V. V. Moshchalkov, and J. a. Sánchez-Gil, "Mode parity-controlled fano- and lorentz-like line shapes arising in plasmonic nanorods," Nano Lett. 14(5), 2322-2329 (2014).

110. A. F. Koenderink, A. Alù, A. Polman, "Shrinking light-based technology," Science 348, 516 (2015).

111. P. Mühlschlegel, H. J. Eisler, O. J. F. Martin, B. Hecht and D. W. Pohl, "Resonant optical antennas," Science 308, 1607-1609 (2005).

112. A. Espinosa-Soria, A. Griol and A. Martínez, "Experimental measurement of plasmonic nanostructures embedded in silicon waveguide gaps," Opt. Express 24, 9592-9601 (2016).

113. Y. D. Chong, L. Ge, H. Cao, A. D. Stone, "Coherent Perfect Absorbers: Time-Reversed Lasers," Phys. Rev. Lett. 105, 053901 (2010).

114. W. Wan, et al. "Time-Reversed Lasing and Interferometric Control of Absorption," Science 331, 889 (2011). 
115. D. G. Baranov, et al. "Coherent perfect absorbers: linear control of light with light," Nat. Rev. Mat. 2, 17064 (2017).

116. J. Zhang, K. F. MacDonald and N. I. Zheludev, "Controlling lightwith-light without nonlinearity," Light Sci. Appl. 1, e18 (2012).

117. X. Fang, K. F. MacDonald and N. I. Zheludev, "Controlling light with light using coherent metadevices: all-optical transistor, summator and invertor," Light Sci. Appl. 4, e292 (2015).

118. X. Fang, et al. "Ultrafast all-optical switching via coherent modulation of metamaterial absorption," Appl. Phys. Lett. 104, 141102 (2014).

119. M. Husnik, et al. "Absolute extinction cross-section of individual magnetic split-ring resonators," Nature Photon. 2, 614 (2008).

120. F. J. Rodriguez-Fortuño, A. Espinosa-Soria and A. Martinez, "Exploiting metamaterials, plasmonics and nanoantennas concepts in silicon Photonics," J. Opt. 18, 123001 (2016).

121. X. Fang, K. F. MacDonald and N. I. Zheludev, "Controlling light with light using coherent metadevices: all-optical transistor, summator and invertor," Light Sci. Appl. 4, e292 (2015).

122. L. Baldacci, S. Zanotto, G. Biasiol, L. Sorba, and A. Tredicucci, "Interferometric control of absorption in thin plasmonic metamaterials: general two port theory and broadband operation," Opt. Express 23, 9202-9210 (2015).

123. U. K. Chettiar, R. Fernandez-Garcia, A. M. Stefan, and N. Engheta, "Enhancement of radiation from dielectric waveguides using resonant plasmonic coreshells," Opt. Express 20, 1610416112 (2012).

124. J. Merlein, "Nanomechanical control of an optical antenna," Nat. Photonics 2, 230-233 (2008).

125. T. B. Hoang and M. H. Mikkelsen, "Broad electrical tuning of plasmonic nanoantennas at visible frequencies," Appl. Phys. Lett. 108, 183107 (2016)

126. K. Y. Bliokh, F. J Rodríguez-Fortuño, F. Nori and A. V. Zayats, "Spin-orbit interactions of light," Nat. Photon. 9, 796-808 (2015).

127. R. M. A. Azzam, I. M. Elminyawi, A. M. J. El-Saba, "General analysis and optimization of the four-detector photopolarimeter," Opt. Soc. Am. 5, 681(1988). 
128. M. R. Foreman, A. Favaro, A. Aiello. "Optimal frames for polarization state reconstruction," Phys. Rev. Lett. 115, 263901, (2015).

129. F. Afshinmanesh, J. S. White, W. Cai, M. L. Brongersma, "Measurement of the polarization state of light using an integrated plasmonic polarimeter," Nanophotonics 1, 125 (2012).

130. Y. B. Xie, Z. Y. Liu, Q. J. Wang, Y. Y. Zhu, X. J. Zhang, "Miniature polarization analyzer based on surface plasmon polaritons," Appl. Phys. Lett. 105, 101107 (2014).

131. D. Wen, F. Yue, S. Kumar, Y. Ma, M. Chen, X. Ren, P. E. Kremer, B. D. Gerardor, M. R. Taghizadeh, G. S. Buller, X. Chen, "Metasurface for characterization of the polarization state of light," Opt. Express 23, 10272 (2015).

132. J. P. Balthasar Mueller, K. Leosson, F. Capasso, "Ultracompact metasurface in-line polarimeter," Optica 3, 42 (2016).

133. A. Pors, M. G. Nielsen, S. I. Bozhevolnyi, "Plasmonic metagratings for simultaneous determination of Stokes parameters," Optica 2, 716 (2015).

134. T. Lepetit, B. Kanté, "Metasurfaces: Simultaneous Stokes parameters," Nature Photon. 9, 709, (2015).

135. E. Maguid, I. Yulevich, D. Veksler, V. Kleiner, M. L. Brongersma, E. Hasman, "Photonic spin controlled multifunctional shared-aperture antenna array," Science 352, 1202 (2016).

136. F. Cardano, L. Marrucci, "Spin-orbit Photonics," Nat. Photonics 9, 776 (2015).

137. J. Lin, J. B. Mueller, Q. Wang, G. Yuan, N. Antoniou, X. C. Yuan, F. Capasso, "Polarization-controlled tunable directional coupling of surface plasmon polaritons," Science 340, 331 (2013).

138. I. Söllner, S. Mahmoodian, S. L. Hansen, L. Midolo, A. Javadi, G. Kiršanske, T. Pregnolato, H. El-Ella, E. H. Lee, J. D. Song, S. Stobbe, P. Lodahl, "Deterministic photon-emitter coupling in chiral photonic circuits," Nat. Nanotechnol. 10, 775 (2015)

139. R. Mitsch, C. Sayrin, B. Albrecht, P. Schneeweiss, A. Rauschenbeutel, "Quantum state-controlled directional spontaneous emission of photons into a nanophotonic waveguide," Nat. Commun. 5, 5713 (2014). 
140. A. M. Beckley, T. G. Brown, M. A. Alonso, "Full Poincaré beams," Opt. Express 18, 10777 (2010).

141. T. Bauer, P. Banzer, E. Karimi, S. Orlov, A. Rubano, L. Marrucci, E. Santamato, R. W. Boyd, G. Leuchs, "Observation of optical polarization Möbius strips, ” Science 347, 964 (2015).

142. R. A. Chipman, "Polarimetry," in Handbook of Optics, M. Bass, ed. The McGraw-Hill Companies, Inc., (2010).

143. A. Aiello, G. Puentes, D. Voigt, and J. P. Woerdman, "Maximumlikelihood estimation of Mueller matrices," Opt. Lett. 31, 817-819 (2006).

144. A. Peinado, A. Lizana, J. Vidal, C. Iemmi, and J. Campos, "Optimization and performance criteria of a Stokes polarimeter based on two variable retarders," Opt. Express 18, 9815-9830 (2010).

145. J. S. Tyo, "Design of optimal polarimeters: maximization of signal-to-noise ratio and minimization of systematic error," Appl. Opt. 41(4), 619-630 (2002).

146. H. Fukuda, K. Yamada, T. Tsuchizawa, T. Watanabe, H. Shinojima, S. I. Itabashi, "Ultrasmall polarization splitter based on silicon wire waveguides," Opt. Express 14, 12401 (2006).

147. R. Ortuño, M. Cortijo and A. Martínez, "Fano resonances and electromagnetically induced transparency in silicon waveguides loaded with plasmonic nanoresonators," J. Opt. 19, 025003 (2017).

148. H. Zhao, et al. "Metawaveguide for Asymmetric Interferometric Light-Light Switching," Phys. Rev. Lett. 117, 193901 (2016)

149. B. Chen et al. "Hybrid Photon-Plasmon Coupling and Ultrafast Control of Nanoantennas on a Silicon Photonic Chip," Nano Lett. 18, 610-617 (2018)

150. Z. J. Wong, et al. "Lasing and anti-lasing in a single cavity," Nat. Photonics 10, 796 (2016).

151. A. E. Miroshnichenko, et al. "Nonradiating anapole modes in dielectric nanoparticles," Nat. Commun. 6, 8069 (2015).

152. A. Alù and N. Engheta, "Achieving transparency with plasmonic and metamaterial coatings," Phys. Rev. E. 72, 016623 (2005). 
153. T. Bauer, S. Orlov, U. Peschel, P. Banzer, G. Leuchs, "Nanointerferometric amplitude and phase reconstruction of tightly focused vector beams," Nature Photonics 8, 23 (2014).

154. B. Redding, S. F. Liew, R. Sarma, H. Cao, "Compact spectrometer based on a disordered photonic chip," Nature Photonics 7, 746 (2013). 


\section{Author's merits}

\section{Journal paper's}

- A. Espinosa-Soria, E. Pinilla-Cienfuegos, F. J. Díaz-Fernández, A. Griol and A. Martínez, "Coherent control of a plasmonic nanoantenna integrated on a silicon chip," ACS Photonics (Accepted, 2018).

- A. Espinosa-Soria, F. J. Rodríguez-Fortuño, A. Griol and A. Martínez, "On-Chip Optimal Stokes Nanopolarimetry Based on Spin-Orbit Interaction of Light," Nano Letters 17(5), 3139-3144 (2017).

- A. Espinosa-Soria, A. Griol and A. Martínez, "Experimental measurement of plasmonic nanostructures embedded in silicon waveguide gaps," Optics Express 24, 9592-9601 (2016).

- F. J. Rodríguez-Fortuño, A. Espinosa-Soria and A. Martínez, "Exploiting metamaterials, plasmonics and nanoantennas concepts in silicon photonics," Journal of Optics 18, 123001 (2016).

- A. Espinosa-Soria and A. Martínez, "Transverse Spinand SpinOrbit Coupling in Silicon Waveguides," IEEE Photonics Technology Letters. 28, 1561-1564 (2016).

- $\quad$ F. Cortés-Juan, A. Espinosa-Soria, J. P. Connolly, G. SánchezPlaza, J. P. Hugonin and P. Sanchis, "Realistic simulation of metal nanoparticles on solar cells," Energy Procedia 84, 204-213 (2015).

\section{Conferences}

- A. Espinosa-Soria, F. J. Rodríguez-Fortuño, A. Griol and A. Martínez, "Stokes Nanopolarimeter Based on Spin-Orbit Interaction of Light," in 11th International Congress on Engineered Material Platforms for Novel Wave Phenomena. Marseille (2017).

- A. Espinosa-Soria, A. Griol and A. Martínez, "Efficiently integrating plasmonic nanostructures into silicon waveguides," in 6th International Topical Meeting on Nanophotonics and Metamaterials. Seefeld (2017). 
- A. Espinosa-Soria, A. Griol and A. Martínez, "Embedding a plasmonic nanoantenna into a silicon waveguide gap: Simulations and experimental demonstration," in 10th International Congress on Advanced Electromagnetic Materials in Microwaves and Optics. Crete (2016).

- A. Espinosa-Soria, F. J. Rodríguez-Fortuño, A. Griol and A. Martínez, "Stokes Polarimetry using Silicon Nanoantennas," in 7th International Conference on Metamaterials, Photonic Crystals and Plasmonics. Torremolinos (2016).

- A. Espinosa-Soria, F. J. Rodríguez-Fortuño, A. Griol and A. Martínez, "Beam polarimetry using silicon nanoantennas," in $V$ Conferencia Española de Nanofotónica. Valencia (2016).

- A. Espinosa-Soria, A. Griol and A. Martínez, "Experimental and numerical demonstration of a plasmonic nanoantenna embedded in a silicon waveguide gap," in $V$ Conferencia Española de Nanofotónica. Valencia (2016).

- A. Espinosa-Soria, F. J. Rodríguez-Fortuño, A. Griol and A. Martínez, "Full Measurement of the Stokes Parameters Using a Subwavelength Silicon On-Chip Polarimeter," in 5th International Conference Smart and Multifunctional Materials, Structures and Systems. Perugia (2016).

- A. Espinosa-Soria, F. J. Rodríguez-Fortuño, A. Griol and A. Martínez, "A subwavelength Stokes polarimeter on a silicon chip," in SPIE Photonics Europe Conference 2016 Nanophotonics VI. Brussels (2016).

- A. Espinosa-Soria, F. J. Rodríguez-Fortuño, A. Griol and A. Martínez, "Nanoantenna-Based Stokes Polarimeter on a Silicon Chip," in Nanolight 2016. Benasque (2016).

- A. Espinosa-Soria, S. Mas, A. Griol, F. J. Rodríguez-Fortuño and A. Martínez, "Full Measurement of the Stokes Parameters of a Light Beam using On-Chip Silicon Nanoantennas," in 9th International Congress on Advanced Electromagnetic Materials in Microwaves and Optics. Oxford (2015).

- A. Martínez, A. Espinosa-Soria, S. Mas, A. Griol and F. J. Rodríguez-Fortuño, "Synthesis and measurement of polarization states with silicon nanoantennas," in ImagineNano 2015 Bringing together Nanoscience \& Nanotechnology. Bilbao (2015). 
- F. J. Rodríguez-Fortuño, D. Puerto, A. Espinosa-Soria, A. Griol and A. Martínez, "Arbitrary Light Polarization Synthesis and Sorting with Silicon Nanoantennas," in 5th International Topical Meeting on Nanophotonics and Metamaterials. Seefeld (2015).

\section{Intellectual property}

\section{PATENT ES2566684B2.}

A. Espinosa-Soria, A. Griol, A. Martínez. Dispositivo y método para medir el estado de polarización de un haz incidente. Universitat Politècnica de València (2016). 


\section{Abstract}

Silicon photonics has become the most important technology in integrated photonic chips production. Its great advantages, including its suitability for large-scale production and low-cost production, as a result of the possibility of using CMOS technology, are sufficient reason to justify its supremacy over other integration platforms. Despite the multiple devices already implemented in this technology, among which include WDM filters or electro-optical modulators, there is still room for improvement, especially in terms of reducing the devices footprint or the creation of new functionalities for the manipulation of light.

Such improvements could be carried out by integrating components with sublambda dimensions arising in the field known as plasmonics. This discipline studies the interaction between light and metals, which is mediated by the existence of waves known as surface plasmons. One of the key properties of plasmons is their ability to confine light well beyond the diffraction limit, which is limiting in the case of silicon photonics. However, losses due to the absorption of metals at optical frequencies prevent their use for guiding light over long distances. Therefore, the benefits of unifying these two worlds becomes evident. By using silicon as the conductive material of the optical signal and the metal as an efficient light interconnector in subwavelength structures, new devices can be created for the manipulation of the properties of light at the nanoscale.

This thesis is focused on the integration of structures with subwavelength dimensions in silicon waveguides and in their application to new functionalities of light manipulation in silicon chips. These nanostructures serve as transducers between guided light and free space radiation, so they can also be termed nanoantennas. To begin with, the guided modes properties in silicon waveguides are described for the correct excitation of the nanoantennas, followed by the demonstration of integration techniques of these nanostructures in these waveguides to increase their interaction efficiency with the guided light. In addition, the coherent control of the absorption and scattering of a metallic nanoantenna integrated in a silicon waveguide is demonstrated. Finally, from the asymmetric positioning of the nanostructure with respect to the waveguide, new polarization manipulation methods are proposed and demonstrated, such as the ability to synthesize desired states of polarization at the nanoscale. This will lead to the theoretical and experimental demonstration of a Stokes nanopolarimeter, based on photon-on-silicon technology, capable of determining the polarization state locally, optimally, and non-destructively, enabling its use for real-time polarization measurements in integrated circuits. 


\section{Resumen}

La fotónica sobre silicio se ha convertido en la tecnología más importante en la producción de chips integrados fotónicos. Sus grandes ventajas, entre las cuales destacan su idoneidad para la fabricación a gran escala y su bajo coste de producción, como resultado de la posibilidad del uso tecnología CMOS, son motivo suficiente para justificar su supremacía sobre otras plataformas de integración. Pese a los múltiples dispositivos ya implementados en dicha tecnología, entre los que cabe destacar filtros WDM o moduladores electroópticos, todavía hay espacio para la mejora, sobre todo en cuanto a la reducción del foot-print de los dispositivos o a la creación de nuevas funcionalidades para la manipulación de la luz.

Dichas mejoras podrían llevarse a cabo mediante la integración de componentes con dimensiones sub-lambda surgidos en el campo conocido como plasmónica. Esta disciplina estudia la interacción entre la luz y los metales, que viene mediada por la existencia de ondas conocidas como plasmones de superficie. Una de las propiedades clave de los plasmones es su capacidad para confinar la luz muy por encima del límite de difracción, lo cual es limitante en el caso de la fotónica sobre silicio. Sin embargo, las pérdidas por absorción de los metales a frecuencias ópticas impiden su uso para el guiado de la luz en grandes distancias. Se hace evidente, por tanto, los beneficios de unificar estos dos mundos. Usando el silicio como material conductor de la señal óptica y el metal como eficiente interactor con la luz en estructuras sub-lambda, se pueden crear nuevos dispositivos para la manipulación de las propiedades de la luz en la nanoescala.

Esta Tesis está centrada en la integración de estructuras con dimensiones sublambda en guías de silicio y en su aplicación a nuevas funcionalidades de manipulación de la luz en chips de silicio. Dichas nanoestructuras sirven de transductores entre la luz guiada y la radiación en espacio libre, por lo que también pueden ser denominadas nanoantenas. Para empezar, se describen las propiedades de los modos guiados en guías de onda de silicio para la correcta excitación de las nanoantenas, seguido de la demostración de técnicas de integración de estas nanoestructuras en las propias guías para aumentar su eficiencia de interacción con la luz guiada. Además, se demuestra el control coherente de la absorción y el scattering de una nanoantenna metálica integrada en una guía de silicio. Por último, a partir del posicionamiento asimétrico de la nanoestructura con respecto a la guía, se proponen y demuestran nuevos métodos de manipulación de la polarización, como la capacidad para sintetizar estados de polarización deseados a escala nanométrica. Esto desembocará en la demostración teórica y experimental de un nanopolarímetro de Stokes, basado en tecnología fotónica sobre silicio, capaz de determinar el estado de polarización de manera local, óptima, y no destructiva, habilitándose su uso para medidas de polarización en tiempo real en circuitos integrados. 


\section{Resum}

La fotònica sobre silici s'ha convertit en la tecnologia més important en la producció de xips integrats fotònics. Els seus grans avantatges, entre les quals destaquen la seva idoneïtat per a la fabricació a gran escala i el seu baix cost de producció, com a resultat de la possibilitat de l'ús tecnologia CMOS, són motiu suficient per justificar la seva supremacia sobre altres plataformes d'integració. Malgrat els múltiples dispositius ja implementats en aquesta tecnologia, entre els quals cal destacar filtres WDM o moduladors electroòptics, encara hi ha espai per a la millora, sobretot quant a la reducció del footprint dels dispositius o a la creació de noves funcionalitats per a la manipulació de la llum.

Aquestes millores podrien portar-se a terme mitjançant la integració de components amb dimensions sub-lambda sorgits en el camp conegut com plasmònica. Aquesta disciplina estudia la interacció entre la llum i els metalls, que ve intervinguda per l'existència d'ones conegudes com plasmons de superfície. Una de les propietats clau dels plasmons és la seva capacitat per confinar la llum molt per sobre del límit de difracció, la qual cosa és limitant en el cas de la fotònica sobre silici. No obstant això, les pèrdues per absorció dels metalls a frequiències òptiques impedeixen el seu ús per al guiat de la llum en grans distàncies. Es fa evident, per tant, els beneficis d'unificar aquests dos mons. Usant el silici com a material conductor del senyal òptic i el metall com eficient interactor amb la llum en estructures sub-lambda, es poden crear nous dispositius per a la manipulació de les propietats de la llum en la nanoescala.

Aquesta Tesi està centrada en la integració d'estructures amb dimensions sublambda en guies de silici i en la seva aplicació a noves funcionalitats de manipulació de la llum en xips de silici. Aquestes nanoestructures serveixen de transductors entre la llum guiada i la radiació en espai lliure, de manera que també poden ser denominades nanoantenes. Per començar, es descriuen les propietats de les maneres guiats en guies d'ona de silici per a la correcta excitació de les nanoantenes, seguit de la demostració de tècniques d'integració d'aquestes nanoestructures en les pròpies guies per augmentar la seva eficiència d'interacció amb la llum guiada. A més, es demostra el control coherent de l'absorció i el scattering d'una nanoantenna metàl-lica integrada en una guia de silici. Finalment, a partir del posicionament asimètric de la nanoestructura respecte a la guia, es proposen i demostren nous mètodes de manipulació de la polarització, com la capacitat per sintetitzar estats de polarització desitjats a escala nanomètrica. Això desembocarà en la demostració teòrica i experimental d'un nanopolarímetre de Stokes, basat en tecnologia fotònica sobre silici, capaç de determinar l'estat de polarització de manera local, òptima, i no destructiva, habilitant el seu ús per a mesures de polarització en temps real en circuits integrats. 